\title{
Small Area Estimation: Informative Sampling and Two-fold Models
}

\author{
by
}

\author{
Golshid Chatrchi
}

A thesis submitted to the Faculty of Graduate and Postdoctoral Affairs in partial fulfillment of the requirements

for the degree of

Doctor of Philosophy

in

Probability and Statistics

Carleton University

Ottawa, Ontario

(C) 2018

Golshid Chatrchi 


\section{Abstract}

Small area estimation (SAE) has become a dynamic field of research over the last couple of decades. The increasing demand for small area estimates in both public and private sectors has opened the door to further investigation of more applied problems. In this thesis, we consider three problems in the context of small area estimation: (i) small area estimation under informative sampling, (ii) small area estimation under unmatched two-fold subarea models, and (iii) variable selection under two-fold subarea model.

In survey sampling, often samples are drawn with unequal probabilities to improve the statistical efficiency of the sampling strategy. When the selection probabilities are correlated with the variables of interest, even after conditioning on the auxiliary information, the sampling scheme is called informative. Under informative sampling, the population model does not hold for the sample. Hence, to avoid biased results, the selection effects should be accounted in the inference procedures. To address this problem, we propose a semi-parametric approach by including a P-spline term of selection probabilities in the model.

The basic area-level model assumes that a linear model relates the small area means to area specific auxiliary information. However, in some SAE applications that deal with logit relationships the associated function is non-linear. In this situation, the linking model and the sampling model do not match and cannot be combined to produce the basic area-level model. We propose an empirical best 
approach to estimation of small subarea parameters under a two-fold subarea-level model consisting of a sampling model and an unmatched linking model.

The main assumption behind standard variable selection methods is that the variable of interest is observed, which is not the case for small area models, where dependent variables are not directly observed, due to the sampling errors. In particular, for a matched two-fold subarea level model, direct estimates of subarea means are measured as proxies for the unknown variable of interest. Applying the standard regression model selection naively may result in inefficient model selection methods. To tackle this issue, we propose a two-step adjustment to the standard variable selection method. 


\section{Acknowledgements}

First and foremost, I would like to express my sincere gratitude to my thesis supervisors, Professor J. N. K. Rao and Professor Song Cai, for their constant guidance, patience and motivation. Indubitably, I would not have been able to complete this thesis without their help. Professor Rao has been my mentor and advisor with endless source of knowledge and experience throughout my graduate studies at Carleton University. From him, not only I learned many aspects of survey sampling and small area estimation, but also valuable life lessons. Professor Cai gave me tremendous support, encouraged me with enlightening guidance and helped me in the writing of this thesis. It has been an honor for me to be their student.

I would also like to thank Professor Yogendra Chaubey, Professor Patrick Farrell, Professor Roland Thomas and Professor Chen Xu for serving as members of my advisory committee and for their valuable comments on my thesis research.

I would like to thank Professor Jason Nielsen who guided me at the the early stage of my Ph.D. study and helped me with the two comprehensive exams. I am also grateful to Professor Laura Dumitrescu from Victoria University of Wellington (New Zealand) for her assistance, valuable suggestions and helpful discussions we had throughout the preparation of this thesis. I would also like to thank my friends, Dr. Yuliya Romanyuk and Dr. Boyan Bejanov for their insightful comments and assistance along the journey to complete this work. 
In addition, I wish to express my deep gratitude to my husband, Amir, for his enduring love and tremendous encouragements and companionship. I am grateful and indebted forever to my parents, Mina and Mostafa, for their endless love and support, without whom I could not have made it here. I would also like to thank my late grandma, for her prayers, encouragement and moral support. Her memory will be with me always. 
"Hardship may dishearten at first, but every hardship passes away. All despair is followed by hope; all darkness is followed by sunshine."

Rumi 


\section{Contents}

Abstract .............................. ii

Acknowledgements ........................ iv

List of Tables . . . . . . . . . . . . . . . . . xi

List of Figures . . . . . . . . . . . . . . . . . . xii

List of Acronyms . . . . . . . . . . . . . . . . . . xiii

1 Introduction 1

1.1 An overview of small area models . . . . . . . . . . . . . . 2

1.1.1 Unit-level models . . . . . . . . . . . . . . . . . . 5

1.1.2 Area-level models . . . . . . . . . . . . . . . . . 6

1.1.3 General linear mixed models . . . . . . . . . . . . . 8

1.2 Model-based estimation . . . . . . . . . . . . . . . 10

1.2.1 Empirical best linear unbiased prediction (EBLUP) method 10

1.2.2 Empirical Bayes (EB) method . . . . . . . . . . . 11

1.2.3 Hierarchical Bayes (HB) method . . . . . . . . . . . 12

1.3 Thesis Outline . . . . . . . . . . . . . . . . . . . . 13

2 Semi-parametric small area estimation under one-stage informa$\begin{array}{ll}\text { tive sampling } & 15\end{array}$ 
2.1 Informative Sampling . . . . . . . . . . . . . . . . . 16

2.2 Small area estimation under informative sampling . . . . . . . . . 17

2.2.1 Pfeffermann and Sverchkov's method . . . . . . . . . . . 19

2.2.2 Verret, Rao and Hidiroglou's method . . . . . . . . . . 20

2.3 P-spline augmentation . . . . . . . . . . . . . . . . . . . . . . . 21

2.3.1 Penalized spline regression . . . . . . . . . . . . 22

2.3.2 $\quad$ P-spline mixed model . . . . . . . . . . . . . . 24

2.4 Proposed method . . . . . . . . . . . . . . . 24

2.5 Mean squared error . . . . . . . . . . . . . . . . . . . 28

2.6 Simulation study . . . . . . . . . . . . . . . . . . . . . 30

2.7 Summary . . . . . . . . . . . . . . . . . . 35

3 Semi-parametric small area estimation under two-stage informa$\begin{array}{ll}\text { tive sampling } & 36\end{array}$

3.1 Two-stage informative sampling . . . . . . . . . . . . . . . 37

3.2 Proposed method . . . . . . . . . . . . . . . . . . . 37

3.2.1 Case I: both stages are informative . . . . . . . . . . 38

3.2.2 Case II: stage 1 informative, stage 2 not informative . . . . 44

3.2.3 Case III: stage 1 not informative, stage 2 informative . . . 46

3.3 Mean squared error . . . . . . . . . . . . . . . 47

3.4 Simulation study . . . . . . . . . . . . . . . . . . 49

3.4.1 Case I: both stages are informative . . . . . . . . . . 49

3.4.2 Case II: stage 1 informative, stage 2 not informative . . . . 53

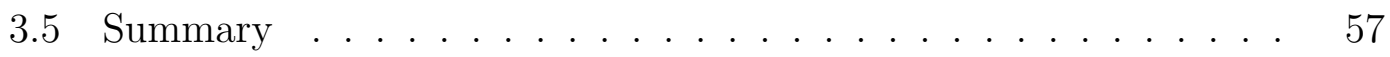

$4 \quad$ EB estimation under unmatched two-fold subarea models $\quad 59$ 
4.1 Unmatched one-fold model . . . . . . . . . . . . . . 60

4.2 Matched two-fold subarea model . . . . . . . . . . . . . 61

4.3 Unmatched two-fold model . . . . . . . . . . . . . . . . . . . . . . 64

4.3.1 BP estimators of subarea parameters $\theta_{i j} \ldots \ldots 6$

4.3.2 Estimation of model parameters . . . . . . . . . . 71

4.3.3 EB estimator .................. 74

4.3.4 Special case I: normal random effects . . . . . . . . . . . 74

4.3.5 Special case II: matched two-fold model with normal random effects . . . . . . . . . . . . 76

4.4 Estimation of mean squared error . . . . . . . . . . . . . . . . 78

4.5 Unmatched three-fold model . . . . . . . . . . . . . . . . . 79

4.6 Simulation Study . . . . . . . . . . . . . . . 85

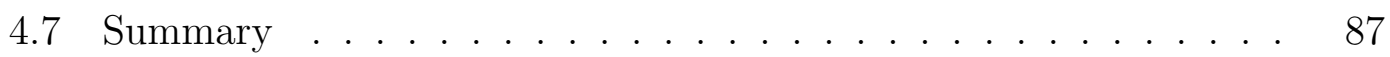

5 Variable Selection under two-fold subarea model 89

5.1 Introduction . . . . . . . . . . . . . . . . . . . 90

5.1.1 Akaike information criterion (AIC) . . . . . . . . 91

5.1.2 Bayesian information criterion (BIC) . . . . . . . . . 92

$5.1 .3 \quad$ Adjusted $R^{2} \ldots \ldots \ldots \ldots$. . . . . . . . . . . 93

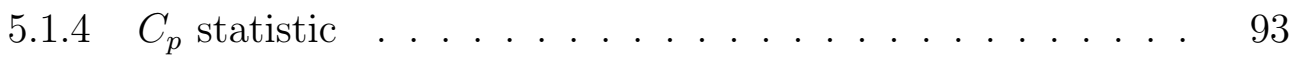

5.2 Variable selection in small area estimation . . . . . . . . . . 94

5.2.1 Lahiri and Suntornchost's paper . . . . . . . . . . . . 94

5.3 Proposed method . . . . . . . . . . . . . . . . 96

5.3.1 Transformation of the linking and sampling models . . . . 97

5.3.2 New variable selection criterion based on $\boldsymbol{\theta}^{\star}$. . . . . . . 100

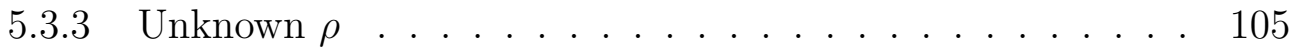


5.4 Simulation study . . . . . . . . . . . . . . . . 107

5.5 Summary . . . . . . . . . . . . . . . . . 114

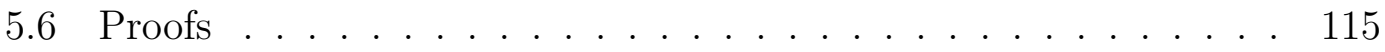

5.6.1 Derivation of (5.16) and (5.17) . . . . . . . . 115

5.6 .2 Proof of $(5.18) \ldots \ldots \ldots \ldots$

5.6.3 Proof of $(5.19) \ldots \ldots \ldots \ldots$

5.7 Appendix

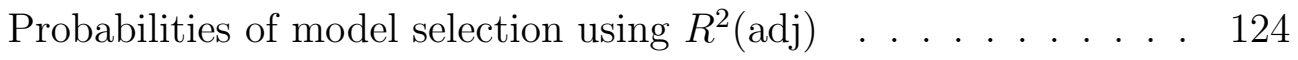

6 Future Research 127

$\begin{array}{lr}\text { Bibliography } & 130\end{array}$ 


\section{List of Tables}

2.1 Average absolute bias and average RMSE of the estimators . . . . 33

2.2 Percent average absolute relative bias of the bootstrap MSE . . . 34

3.1 Average absolute bias and average RMSE for Case I . . . . . . . . 52

3.2 Average absolute bias and average RMSE . . . . . . . . . . . 55

$3.3 \overline{\% A R B}(\%)$ and MSE of bootstrap MSE estimator of EBLUP . . 57

4.1 EB for subarea means under unmatched two fold-models . . . . . 87

5.1 Probabilities of model selection using $A I C_{\text {naive } 1}$. . . . . . . . . . 111

5.2 Probabilities of model selection using $A I C_{\text {naive } 2} \ldots \ldots$. . . . . . 112

5.3 Probabilities of model selection using $A I C_{\text {new }} \ldots$. . . . . . . . . 113

5.4 Probabilities of model selection using $R^{2}(\operatorname{adj})_{\text {naive } 1} \quad$. . . . . . . . 124

5.5 Probabilities of model selection using $R^{2}(\operatorname{adj})_{\text {naive } 2} \quad$. . . . . . . . 125

5.6 Probabilities of model selection using $R^{2}(a d j)_{n e w} \quad \ldots . . . .126$ 


\section{List of Figures}

2.1 Example: piecewise quadratic polynomial with 10 knots . . . . . 23

4.1 Two-fold subarea model: representation of areas and subareas . . 67

5.1 Percentage of selecting the optimal model using adjusted R-squared 114 


\section{List of Acronyms}

\begin{tabular}{ll}
\hline Acronyms & Definition \\
\hline \hline AB & Absolute Bias \\
AIC & Akaike Information Criterion \\
ANOVA & Analysis of Variance \\
ARB & Absolute Relative Bias \\
BIC & Bayesian Information Criterion \\
BLUE & Best Linear Unbiased Estimator \\
BLUP & Best Linear Unbiased Prediction \\
BP & Best Predictor \\
EB & Empirical Best \\
CD & Census Division \\
CDF & Cumulative Distribution Function \\
CSD & Census Subdivision \\
EBLUP & Empirical Best Linear Unbiased Prediction \\
EM & Expectation-Maximization \\
GLS & Generalized Least Square \\
HB & Hierarchical Bayes \\
MC & Monte Carlo \\
MCMC & Markov Chain Monte Carlo \\
ML & Maximum Likelihood \\
\hline
\end{tabular}




\begin{tabular}{ll} 
Acronyms & Definition \\
\hline \hline MSE & Mean Squared Error \\
MST & Mean of Squared Total \\
NPEB & Non-Parametric Empirical Bayes \\
OLS & Ordinary Least Square \\
PDF & Probability Density Function \\
PEB & Parametric Empirical Bayes \\
PPS & Probability Proportional to Size \\
PSU & Primary Sampling Unit \\
REML & Restricted Maximum Likelihood \\
RMSE & Root Mean Square Error \\
SAE & Small Area Estimation \\
SNP & Semi-Nonparametric \\
SSE & Sum of Squares of Error \\
SST & Total Sum of Squares \\
SRS & Simple Random Sampling \\
SSU & Secondary Sampling Unit \\
WLS & Weighted Least Squares \\
&
\end{tabular}




\section{Chapter 1}

\section{Introduction}

Sample surveys are the most effective tool for obtaining information from large populations. In general, they are more timely, less expensive and easier to monitor than a census. Official statistical agencies and other survey institutes are widely using sample surveys to produce different statistical indicators. In particular, Statistics Canada conducts more than 300 surveys that cover 32 different subject matter fields every five years.

Aside from providing estimates for the whole population, it is often of interest to provide estimates for sub-populations or domains. However, it may not be financially or operationally feasible to take a large enough sample size and produce good quality direct estimates for small domains. Small Area Estimation (SAE) theory, which dates back to the eleventh century in England and seventeenth century in Canada (Brackstone, 1987), has mainly been developed to tackle this issue by making use of indirect estimates. A comprehensive account of small area estimation methods and main developments in this field can be found in Rao and Molina (2015). In this thesis, we concentrate on three topics in the context of 
small area estimation. Before introducing the objectives of this dissertation and the considered problems, we present an overview of small area models as well as some of the most commonly used techniques in the next sections.

\subsection{An overview of small area models}

In design-based inference, survey weights are used to provide survey estimates. These estimates are called direct estimates as they are based on the sample information only. The associated inference relies on the sampling design of the survey. A direct estimator may also use some known auxiliary information related to the variable of interest. The theory of design-based inference is covered in many sampling textbooks such as Cochran (2007), Särndal et al. (2003), and Lohr (2009). For large sub-populations, direct estimators can be used to produce reliable estimates. However, for small domains, using direct estimators can result in inaccurate estimates due to inadequate sample sizes or no sample at all. The term small domain or small area refers to any subpopulation for which we cannot produce reliable direct estimates due to small or even zero domain-specific sample size. The domain of interest could be defined as a geographical region (e.g. a county, a municipality or a census sub-division) or a demographic group (e.g. sex $\times$ age-group), as well as a demographic group within a geographic region.

In practice, direct estimates may not be available due to different reasons. For example, a sampling design which intends to provide estimates for large areas like provinces cannot produce reliable estimates for small areas of interest (Jiang and Lahiri, 2006). In this situation, increasing the sample size (i.e., oversampling) is not an ideal or feasible solution. More precisely, we cannot allocate a large enough 
sample to each small domain due to time and budget constraints. Moreover, sometimes domains of interest are modified after the collection phase of the survey (e.g., a priority change from provincial estimates to sub-provincial estimates after the collection of data).

Small area estimation uses indirect estimators that borrow strength from other related domains through linking models, by using auxiliary data sources like census or administrative data. Indirect domain estimators can be divided into two groups: traditional estimators and model-based estimators.

Traditional estimators are based on implicit linking models, including synthetic estimators, composite estimators and James-Stein estimators (James and Stein, 1961). These three methods are summarized briefly below. More information can be found in Chapter 3 of Rao and Molina (2015).

The synthetic estimators rely on the assumption that small areas have the same characteristics as the large areas. Under this assumption, a reliable direct estimator for a large area (that covers many small areas) is used to derive an indirect estimator for a small area (Gonzalez, 1973). Different types of synthetic estimators are discussed in Section 3.2 of Rao and Molina (2015). For example, if direct estimates $\hat{Y}_{i}$ of area totals $Y_{i}$ and related area-level auxiliary variables $x_{i 1}, \cdots, x_{i p}$ are available for $m$ out of $M$ areas $(i=1, \cdots, m)$, then a linear regression can be fitted to the data $\left(\hat{Y}_{i}, x_{i 1}, \cdots, x_{i p}\right)$ using least squares. The regression-synthetic estimators for all $M$ areas are given by

$$
\tilde{Y}_{i}=\hat{\beta}_{0}+\hat{\beta}_{1} x_{i 1}+\cdots+\hat{\beta}_{p} x_{i p}, \quad i=1, \cdots, M,
$$


where $\hat{\beta}_{0}, \hat{\beta}_{1}, \cdots, \hat{\beta}_{p}$ are estimated regression coefficients from the $m$ sampled areas. Indeed, the regression-synthetic estimator (1.1) can be used for non-sampled areas $(i=m+1, \cdots, M)$. Note that estimator (1.1) can be highly biased if the underlying model assumptions are not valid.

Composite estimators are another type of indirect estimators that use a weighted average of a synthetic estimator and a direct estimator. The goal is to balance the potential bias of the synthetic estimators against the instability of the direct estimator. The composite estimator of small area total $Y_{i}$ can be written as

$$
\hat{Y}_{i C}=\phi_{i} \hat{Y}_{i 1}+\left(1-\phi_{i}\right) \hat{Y}_{i 2}
$$

where $\hat{Y}_{i 1}$ is a direct estimator and $\hat{Y}_{i 2}$ is a synthetic estimator. The optimal weight $\phi_{i}$ is obtained by minimizing the design MSE of the composite estimator.

Another approach to composite estimation is to use a common weight for all areas $\phi_{i}=\phi$, and then minimize the total MSE to obtain $\hat{\phi}^{*}$ (the optimal weight). The composite estimator based on $\hat{\phi}^{*}$ is similar to the well-known James-Stein (JS) (James and Stein, 1961) estimator. Several features of the the JS method are discussed in Section 3.4 of Rao and Molina (2015).

Traditional indirect estimators mentioned above are based on implicit models that provide a link to related small area via auxiliary information. On the other hand, model-based estimators rely on explicit linking models that make specific allowance for between-area variation. In particular, mixed models include random area-specific effects that accounts for between-area variation beyond that explained by supplementary data in the model. As mentioned in Rao and Molina (2015), explicit models have several advantages such as providing area-specific measure of precision, and the possibility of using model diagnostics for finding the 
optimal model. The most commonly used techniques for small area estimation are the empirical best linear unbiased prediction (EBLUP), the empirical Bayes (EB) procedures and the hierarchical Bayes (HB). These estimators have some advantages for small area estimation. For instance, optimal estimators can be obtained under assumed models with respect to the nature of response variable. Small area models can be classified into two types, depending on the availability of data:

(i) Unit-level models that relate the unit values of the study variable to unitspecific covariates,

(ii) Area-level models that relate small area direct estimators to area-specific covariates.

Both unit-level models and area-level models are studied in this thesis. In chapters 2 and 3, the focus is on unit-level models while in chapter 4 and 5 we consider an extension of the basic area-level model. These models and their properties are reviewed in the following subsections.

We follow the notation and terminology of Rao and Molina's book (2015).

\subsubsection{Unit-level models}

Unit-level models assume that the variable of interest, $y_{i j}$, is related to unit-specific auxiliary data, $\mathbf{x}_{i j}=\left(x_{i j 1}, x_{i j 2}, \cdots, x_{i j p}\right)^{T}$, through the basic nested error linear regression model. The population model is given by:

$$
y_{i j}=\mathbf{x}_{i j}^{T} \boldsymbol{\beta}+v_{i}+e_{i j}, \quad j=1, \cdots, N_{i}, \quad i=1, \cdots, M,
$$


where $M$ is the total number of areas, $v_{i}$ 's are the area-specific random effects, which are assumed to be independent and identically distributed (iid) random variables with mean zero and variance $\sigma_{v}^{2}$. The sampling errors, $e_{i j}$, are assumed to be iid random variables with mean zero and variance $k_{i j}^{2} \sigma_{e}^{2}$. It is assumed that the $v_{i}$ 's and $e_{i j}$ 's are independent. Also, normality of the $v_{i}$ 's and $e_{i j}$ 's is often assumed. Although the model assumes that unit-specific covariates are available for all the $N_{i}$ population elements in each small area, it is often sufficient to have known population means $\overline{\mathbf{X}}_{i}$.

Assuming that all areas are sampled $(M=m)$ and the sampling within areas is non-informative (i.e., the model holding for the population values also holds for the sample data), we can use the sample part of the population model to make an inference about the population mean $\bar{Y}_{i}=\frac{1}{N_{i}} \sum_{j} y_{i j}$. The sample model is given by:

$$
y_{i j}=\mathbf{x}_{i j}^{T} \boldsymbol{\beta}+v_{i}+e_{i j}, \quad j=1, \cdots, n_{i}, \quad i=1, \cdots, m
$$

The non-informative assumption is further discussed in Chapter 2 and Chapter 3. The model (1.2) can be written in a matrix form

$$
\mathbf{y}_{i}=\mathbf{X}_{i} \boldsymbol{\beta}+v_{i} \mathbf{1}_{n_{i}}+\mathbf{e}_{i}, \quad i=1, \cdots, m
$$

where $\mathbf{y}_{i}=\left(y_{i 1}, \cdots, y_{i n_{i}}\right)^{T}, \mathbf{X}_{i}=\left(\mathbf{x}_{i 1}, \cdots, \mathbf{x}_{i n_{i}}\right)^{T}$, and $\mathbf{e}_{i}=\left(\mathbf{e}_{i 1}, \cdots, \mathbf{e}_{i n_{i}}\right)^{T}$.

\subsubsection{Area-level models}

In general, area-level models are used when unit-level data are not available. A basic area-level model, known as the Fay-Herriot model (Fay and Herriot, 1979), assumes that the area mean, $\theta_{i}$, is related to area-specific auxiliary data, $\mathbf{z}_{i}=$ 
$\left(z_{i 1}, z_{i 2}, \cdots, z_{i p}\right)^{T}$, via a linear model.

$$
\theta_{i}=\mathbf{z}_{i}^{T} \boldsymbol{\beta}+b_{i} v_{i}, \quad i=1, \ldots, M
$$

where $M$ is the number of areas, $b_{i}$ 's are known positive constants, $\boldsymbol{\beta}=$ $\left(\beta_{1}, \cdots, \beta_{p}\right)^{T}$ is a $p \times 1$ vector of regression coefficients and $v_{i}$ denotes the areaspecific random effect. It is assumed that $v_{i}$ 's are independent and identically distributed with $E_{m}\left(v_{i}\right)=0, V_{m}\left(v_{i}\right)=\sigma_{v}^{2}$, where $E_{m}$ and $V_{m}$ represent model expectation and variance, respectively. Suppose that $m$ areas out of $M$ are selected in the sample. We assume that sampled areas follow the population model (1.4):

$$
\theta_{i}=\mathbf{z}_{i}^{T} \boldsymbol{\beta}+b_{i} v_{i}, \quad i=1, \ldots, m
$$

We further assume that direct estimators $\hat{\theta}_{i}$ of $\theta_{i}$ are available.

$$
\hat{\theta}_{i}=\theta_{i}+e_{i}, \quad i=1, \cdots, m,
$$

where $e_{i}$ 's are sampling errors with $E_{p}\left(e_{i}\right)=0$ and $V_{p}\left(e_{i}\right)=\psi_{i}$, where $E_{p}$ and $V_{p}$ denote design expectation and variance, respectively.

Equations (1.5) and (1.6) are called the linking model and the sampling model, respectively. The basic area-level model is obtained by combining (1.5) and (1.6):

$$
\hat{\theta}_{i}=\mathbf{z}_{i}^{T} \boldsymbol{\beta}+b_{i} v_{i}+e_{i}, \quad i=1, \ldots, m
$$

It is assumed that $v_{i}$ 's, model errors, and $e_{i}^{\prime} s$, design errors, are independent. Moreover, it is usually assumed that $b_{i}=1$. 


\subsubsection{General linear mixed models}

The two basic small area models, the area-level model and the unit-level model, and their extensions can be presented as a general linear mixed model with block diagonal covariance structure. The term linear mixed model denotes the mixture of fixed and random effects in a linear model. A general linear mixed model is given by

$$
\mathbf{y}=\mathbf{X} \boldsymbol{\beta}+\mathbf{Z} \mathbf{v}+\mathbf{e}
$$

where $\mathbf{y}$ is the $n \times 1$ vector of sample units, $\mathbf{X}$ is the $n \times p$ known matrix, $\mathbf{Z}$ is the $n \times h$ known matrix of full rank ( $h$ is the number of random effects in vector $\mathbf{v}$ ), $\boldsymbol{\beta}$ is a $p \times 1$ vector of unknown fixed effects, $\mathbf{v}$ and $\mathbf{e}$ are independent random vectors with mean zero and covariance matrices $\mathbf{G}$ and $\mathbf{R}$ that depend on some variance parameters, $\boldsymbol{\sigma}=\left(\sigma_{1}^{2}, \ldots, \sigma_{q}^{2}\right)$. We further assume that $\boldsymbol{\sigma}$ belongs to a specified subset of Euclidean $q$-space such that $\operatorname{Var}(\mathbf{y})=\mathbf{V}=\mathbf{V}(\boldsymbol{\sigma})=\mathbf{R}+\mathbf{Z G Z}^{T}$ is nonsingular for all $\boldsymbol{\sigma}$ belonging to the subset, where $\operatorname{Var}(\mathbf{y})$ denotes the variancecovariance matrix of $\mathbf{y}$. As stated by McCulloch and Searle (2004), fixed effects are introduced to model the mean of $\mathbf{y}$ and the random effects are considered to control the variance-covariance of $\mathbf{y}$.

The goal is to estimate a linear combination of fixed effects $\boldsymbol{\beta}$ and realized values of $\mathbf{v}$ in the form of $\mu=\mathbf{l}_{\mathbf{1}}^{T} \boldsymbol{\beta}+\mathbf{l}_{\mathbf{2}}{ }^{T} \mathbf{v}$, for constant $\mathbf{l}_{\mathbf{1}}$ and $\mathbf{l}_{\mathbf{2}}$.

A special case of model (1.8) with a block diagonal covariance structure covers many small area models. A block diagonal matrix is a square matrix, A, whose diagonal elements are square matrices, $A_{k}$, and it is denoted by $\mathbf{A}=\operatorname{diag}\left(A_{i}\right)_{1 \leq i \leq k}$.

For (1.8) we have: $\mathbf{y}=\operatorname{col}_{1 \leq i \leq m}\left(\mathbf{y}_{i}\right), \quad \mathbf{X}=\operatorname{col}_{1 \leq i \leq m}\left(\mathbf{X}_{i}\right), \quad \mathbf{Z}=\operatorname{diag}_{1 \leq i \leq m}\left(\mathbf{Z}_{i}\right)$, $\mathbf{v}=\operatorname{col}_{1 \leq i \leq m}\left(\mathbf{v}_{i}\right), \quad \mathbf{e}=\operatorname{col}_{1 \leq i \leq m}\left(\mathbf{e}_{i}\right)$, where $m$ is the number of small areas, $\mathbf{y}_{i}$ 
is the $n_{i} \times 1$ vector of sample observations in the $i^{t h}$ area, $\mathbf{X}_{i}$ is a $n_{i} \times p$ matrix of auxiliary information in the $i^{\text {th }}$ area and $\mathbf{Z}_{i}$ is a $n_{i} \times h_{i}$ matrix with $\sum_{i=1}^{k} n_{i}=n$ and $\sum_{i=1}^{k} h_{i}=h$.

The covariance matrix $\mathbf{V}$ has the block diagonal structure:

$\mathbf{V}=\operatorname{diag}_{1 \leq i \leq m}\left(\mathbf{V}_{i}\right)=\operatorname{diag}_{1 \leq i \leq m}\left(\mathbf{R}_{i}+\mathbf{Z}_{i} \mathbf{G}_{i} \mathbf{Z}_{i}^{T}\right)$, where $\mathbf{R}=\operatorname{diag}_{1 \leq i \leq m}\left(\mathbf{R}_{i}\right)$ and $\mathbf{G}=\operatorname{diag}_{1 \leq i \leq m}\left(\mathbf{G}_{i}\right)$.

The unit-level model (1.3) is a special case of the general linear mixed models with a block diagonal covariance structure. The variance-covariance matrix of $\mathbf{y}_{i}$ can be written as

$$
V_{i}=V\left(\mathbf{y}_{i}\right)=\mathbf{R}_{i}+\sigma_{v}^{2} \mathbf{1}_{n_{i}} \mathbf{1}_{n_{i}}^{T},
$$

where $\mathbf{R}_{i}=\sigma_{e}^{2} \operatorname{diag}_{1 \leq j \leq n_{i}}\left(k_{i j}^{2}\right)$.

Similarly, the area-level model (1.7) is a special case of the general linear mixed models with a block diagonal covariance structure. More precisely, (1.7) can be written as follows

$$
\mathbf{y}_{i}=\mathbf{X}_{i} \boldsymbol{\beta}+\mathbf{Z}_{i} \mathbf{v}_{i}+\mathbf{e}_{i}
$$

where $\mathbf{y}_{i}=\hat{\theta}_{i}, \mathbf{X}_{i}=\mathbf{z}_{i}^{T}, \mathbf{Z}_{i}=b_{i}, \mathbf{v}_{i}=v_{i}, \mathbf{e}_{i}=e_{i}$ and $\boldsymbol{\beta}=\left(\beta_{1}, \cdots, \beta_{p}\right)^{T}$. The variance-covariance of $\mathbf{y}_{i}$ is

$$
V_{i}=V\left(\mathbf{y}_{i}\right)=b_{i}^{2} \mathbf{G}_{i}+\mathbf{R}_{i},
$$

where $\mathbf{G}_{i}=\sigma_{v}^{2}$ and $\mathbf{R}_{i}=\psi_{i}$. 


\subsection{Model-based estimation}

Model-based indirect estimators are obtained based on a model that incorporates related areas in the estimation process. The most commonly used techniques for small area estimation are the empirical best linear unbiased prediction (EBLUP), the empirical Bayes (EB) and the hierarchical Bayes (HB) procedures.

\subsubsection{Empirical best linear unbiased prediction (EBLUP) method}

For the general linear mixed model (1.8) with block diagonal covariance structure, the best linear unbiased prediction (BLUP) estimator of $\mu_{i}=\mathbf{l}_{i}^{T} \boldsymbol{\beta}+\mathbf{m}_{i}^{T} \mathbf{v}_{i}$ is

$$
\tilde{\mu}_{i}\left(\boldsymbol{\sigma}, \mathbf{y}_{i}\right)=\tilde{\mathbf{l}}_{i}^{T} \tilde{\boldsymbol{\beta}}+\mathbf{m}_{i}^{T} \mathbf{G}_{i} \mathbf{Z}_{i}^{T} \mathbf{V}_{i}^{-1}\left(\mathbf{y}_{i}-\mathbf{X}_{i} \tilde{\boldsymbol{\beta}}\right)
$$

where

$$
\tilde{\boldsymbol{\beta}}=\tilde{\boldsymbol{\beta}}(\sigma)=\left(\sum_{i=1}^{m} \mathbf{X}_{i}^{T} \mathbf{V}_{i}^{-1} \mathbf{X}_{i}\right)^{-1}\left(\sum_{i=1}^{m} \mathbf{X}_{i}^{T} \mathbf{V}_{i}^{-1} \mathbf{y}_{i}\right)
$$

is the generalized least square (GLS) estimator of $\boldsymbol{\beta}$ Henderson (1975).

Equation (1.9) depends on an unknown vector of variance components, $\boldsymbol{\sigma}$, we replace $\boldsymbol{\sigma}$ by an estimator $\hat{\boldsymbol{\sigma}}$ to obtain the empirical BLUP (EBLUP) estimator $\hat{\mu}_{i}=\tilde{\mu}_{i}(\hat{\boldsymbol{\sigma}}, \mathbf{y})$. Assuming normality, maximum likelihood (ML) or restricted maximum likelihood (REML) methods (Hocking and Kutner, 1975) can be used to estimate $\boldsymbol{\sigma}$.

For the unit-level model (1.3), the BLUP of $\mu_{i}=\overline{\mathbf{X}}_{i} \boldsymbol{\beta}+v_{i}$ is given by:

$$
\tilde{\mu}_{i}\left(\boldsymbol{\sigma}, \mathbf{y}_{i}\right)=\overline{\mathbf{X}}_{i}^{T} \tilde{\boldsymbol{\beta}}+\gamma_{i}\left(\overline{\mathbf{y}}_{i a}-\overline{\mathbf{x}}_{i a}^{T} \tilde{\boldsymbol{\beta}}\right),
$$


where

$$
\tilde{\boldsymbol{\beta}}=\left(\sum_{i=1}^{k} \mathbf{X}_{i}^{T} \mathbf{V}_{i}^{-1} \mathbf{X}_{i}\right)^{-1}\left(\sum_{i=1}^{k} \mathbf{X}_{i}^{T} \mathbf{V}_{i}^{-1} \mathbf{y}_{i}\right)
$$

$\gamma_{i}=\sigma_{v}^{2} /\left(\sigma_{v}^{2}+\sigma_{e}^{2} / a_{i .}\right), \overline{\mathbf{y}}_{i a}=\sum_{j=1}^{n_{i}} a_{i j} y_{i j} / a_{i .}, \overline{\mathbf{x}}_{i a}=\sum_{j=1}^{n_{i}} a_{i j} \mathbf{x}_{i j} / a_{i .}$ with $a_{i j}=k_{i j}^{-2}$ and $a_{i .}=\sum_{j=1}^{n_{i}} a_{i j}$.

Similarly, under the area-level model, using (1.9) the BLUP estimator of $\mu_{i}$ is given by:

$$
\tilde{\mu}_{i}\left(\boldsymbol{\theta}, \mathbf{y}_{i}\right)=\mathbf{z}_{i}^{T} \tilde{\boldsymbol{\beta}}+\gamma_{i}\left(\hat{\theta}_{i}-\mathbf{z}_{i}^{T} \tilde{\boldsymbol{\beta}}\right)
$$

where

$$
\gamma_{i}=\sigma_{v}^{2} b_{i}^{2} /\left(\sigma_{v}^{2} b_{i}^{2}+\psi_{i}\right)
$$

and

$$
\tilde{\boldsymbol{\beta}}=\left(\sum_{i=1}^{m} \mathbf{z}_{i} \mathbf{z}_{i}^{T} /\left(\sigma_{v}^{2} b_{i}^{2}+\psi_{i}\right)\right)^{-1}\left(\sum_{i=1}^{m} \mathbf{z}_{i} \hat{\theta}_{i} /\left(\sigma_{v}^{2} b_{i}^{2}+\psi_{i}\right)\right)
$$

and the EBLUP of $\mu_{i}$ is given by $\hat{\mu}_{i}=\tilde{\mu}_{i}(\hat{\boldsymbol{\sigma}})$, where $\hat{\boldsymbol{\sigma}}=\left(\sigma_{e}, \sigma_{v}\right)^{T}$.

\subsubsection{Empirical Bayes (EB) method}

The Empirical Bayes (EB) approach consists of three steps. First, the posterior density, $f(\boldsymbol{\mu} \mid \mathbf{y}, \boldsymbol{\lambda})$ of the parameter of interest, $\boldsymbol{\mu}$ is found by using the conditional density $f\left(\mathbf{y} \mid \boldsymbol{\mu}, \lambda_{1}\right)$ and $f\left(\boldsymbol{\mu} \mid \lambda_{2}\right)$, where $\boldsymbol{\lambda}=\left(\lambda_{1}, \lambda_{2}\right)^{T}$ are model parameters. Second, the model parameters $\boldsymbol{\lambda}$ are estimated from the marginal density $f(\mathbf{y} \mid \boldsymbol{\lambda})$. Third, inferences about $\boldsymbol{\mu}$ are made using the estimated posterior density $f(\boldsymbol{\mu} \mid \mathbf{y}, \hat{\boldsymbol{\lambda}})$, where $\hat{\boldsymbol{\lambda}}$ is an estimator of $\boldsymbol{\lambda}$. It should be noted that sometimes a prior density is assumed for model parameters to derive the estimator and associated measures of variability only (Morris, 1983). The advantage of the EB approach, 
which uses Bayes theory, is that it can be applied to binary, count data and normal linear mixed models.

In the parametric empirical Bayes (PEB) approach, a parametric form is assumed for the distribution of $\boldsymbol{\mu}$. On the contrary, the non-parametric empirical Bayes (NPEB) method does not choose the form of the prior distribution of $\boldsymbol{\mu}$. The semi-nonparametric (SNP) representations of the density of $\boldsymbol{\mu}$ are also studied in the EB inference. The EB methodology is presented in detail in Maritz and Lwin (1989) and Carlin and Louis (2008). Also, in the context of small area estimation, the EB method and related techniques are presented in Chapter 9 of Rao and Molina (2015).

\subsubsection{Hierarchical Bayes (HB) method}

Similar to the EB approach, Hierarchical Bayes (HB) method can handle models for binary and count data as well as linear mixed models. In HB approach, a subjective prior distribution of model parameters is assumed. The goal is to obtain the posterior distribution of a variable of interest given the data. The inference is based on $f(\boldsymbol{\mu} \mid \boldsymbol{\lambda})$, where $\boldsymbol{\lambda}$ denotes the model parameters. The challenge in HB approach is to specify a subjective prior $f(\boldsymbol{\lambda})$ on the model parameters. Priors on $\boldsymbol{\lambda}$ can be informative or non-informative, depending on the availability of substantial prior information like a previous study.

By Bayes theorem, we have

$$
f(\boldsymbol{\mu}, \lambda \mid \mathbf{y})=\frac{f(\mathbf{y}, \boldsymbol{\mu} \mid \lambda) f(\boldsymbol{\lambda})}{f_{1}(\mathbf{y})}
$$


where $f_{1}(\mathbf{y})$ is the marginal density of $\mathbf{y}$, given by

$$
f_{1}(\mathbf{y})=\int f(\mathbf{y}, \boldsymbol{\mu} \mid \boldsymbol{\lambda}) f(\boldsymbol{\lambda}) d \boldsymbol{\mu} d \boldsymbol{\lambda}
$$

The posterior density $f(\boldsymbol{\mu} \mid \mathbf{y})$ is obtained as follows

$$
\begin{aligned}
f(\boldsymbol{\mu} \mid \mathbf{y}) & =\int f(\boldsymbol{\mu}, \boldsymbol{\lambda} \mid \mathbf{y}) d \boldsymbol{\lambda} \\
& =\int f(\boldsymbol{\mu} \mid \mathbf{y}, \boldsymbol{\lambda}) f(\boldsymbol{\lambda} \mid \mathbf{y}) d \boldsymbol{\lambda}
\end{aligned}
$$

It is worth mentioning that the evaluation of $f(\boldsymbol{\mu} \mid \mathbf{y})$ and associated posterior properties such as $E(h(\boldsymbol{\mu}) \mid \mathbf{y})$ need multi-dimensional integrations, which is usually a computationally intensive process. This impacted the ease of applying HB methods in complex problems. However, with the development of Markov chain Monte Carlo (MCMC) methods, computations are done more efficiently and HB methods have become more popular recently. The HB method is discussed in detail in Chapter 10 of Rao and Molina (2015).

\subsection{Thesis Outline}

This dissertation consists of six chapters. Chapters 3 to 5 cover three problems in the context of small area estimation: (i) small area estimation under informative sampling, (ii) empirical best estimation under unmatched two-fold subarea models, and (iii) variable selection under two-fold subarea model. The common point between these topics is that the motivation behind all of them lies in practical needs of survey statisticians. 
We study small area estimation under one-stage and two-stage informative sampling in Chapter 2 and Chapter 3. Estimation under unmatched two-fold subarea models is studied in Chapter 4. In Chapter 5, we study variable selection methods under two-fold subarea model and propose a two-step adjustment to the standard variable selection methods. Chapter 6 gives a brief summary and outlines some topics for future research. 


\section{Chapter 2}

\section{Semi-parametric small area estimation under one-stage informative sampling}

In model-based small area estimation, an explicit model is assumed to enhance the efficiency of the estimation of the small area means. However, if the sampling design is informative, the population model may not hold for the sample and standard model-based estimators may be biased. In the next two chapters, we propose a semi-parametric approach by including a P-spline term of selection probabilities in the model to account for the informativeness of the sampling design. In this chapter, we study one-stage sampling and assume that all areas are sampled $(m=M)$, while the next chapter considers the case of two-stage sampling, where not all areas are sampled $(m<M)$.

Before introducing the idea, we briefly review informative sampling and the existing methods in the literature in the first few sections of this chapter. We will 
discuss the proposed method in Section 2.4. The results of a simulation study are reported in Section 2.6, and concluding remarks are given in Section 2.7.

\subsection{Informative Sampling}

Simple random sampling (SRS) is the simplest sampling technique, in which the probability that each unit will be sampled is the same. However, it is not the most efficient sampling design and it is rarely used in practice. Hence, in survey sampling, often samples are drawn with unequal probabilities to improve the statistical efficiency of the sampling strategy. When the selection probabilities are correlated with the variables of interest, even after conditioning on the auxiliary information (or covariates), the sampling scheme is called informative. In this situation, assuming that the model holds for the sample may lead to biased estimates. We will explain this through an example. Assume that we are interested in estimating the total number of workplace injuries in a given city. Typically, the number of workplace injuries depends on the type of industries and the size of firms. Thus, we give larger establishments with historically higher injury rates a greater chance to appear in the sample. The resulting sample will contain a larger proportion of establishments with higher injury rates compared to the population. In this example, one might be interested in analyzing the relationship between the number of workplace injuries and some other variables (e.g., average number of employees in each shift) through a model. The results of the analysis can be seriously biased, if the information about the sampling scheme is not considered.

In other words, when the sample distribution differs from the population distribution the sampling becomes informative, and the sampling scheme should be 
accounted at the inference step. Indeed, for such sampling designs, the model holding for the population values no longer holds for the sample data. Let $U$ denote a finite population. In mathematical terms, for any measurable set " $A$ " we have

$$
P\left(y_{k} \in A \mid x_{k}, I_{k}=1\right) \neq P\left(y_{k} \in A \mid x_{k}\right),
$$

where $\left(x_{k}, y_{k}\right), k \in U$, are values of the finite population and $I_{k}$ takes value 1 if unit $k$ is sampled and 0 otherwise. The left side of (2.1) is the sample model and the right side is the population model. For non-informative sampling design (like SRS), the right side and the left side of equation (2.1) are equal (Kim and Skinner, 2013).

\subsection{Small area estimation under informative sampling}

As mentioned earlier, the objective of small area estimation is to provide estimates of population totals or means for small sub-population or areas. We assume that the population of interest, $U$, consists of $M$ distinct areas with $N_{i}$ units in the $i^{\text {th }}$ area $(i=1, \ldots, M)$. If unit specific data $\mathbf{x}_{i j}$ are available for each element $j$ in area $i$, then the unit-level model is the appropriate setting, assuming that $y_{i j}$ is related to $\mathbf{x}_{i j}$ through a basic nested error linear regression model:

$$
y_{i j}=\mathbf{x}_{i j}^{T} \boldsymbol{\beta}_{0}+v_{i}+e_{i j} \quad i=1, \ldots, M, \quad j=1, \ldots, N_{i},
$$

where $\mathbf{x}_{i j}$ is the covariate related to $y_{i j}, v_{i} \sim N\left(0, \sigma_{v}^{2}\right)$ is the area effect, and $e_{i j} \sim N\left(0, \sigma_{e}^{2}\right)$ denotes the error term. 
In area $i$, we draw a sample of $n_{i}$ units with selection probabilities of $p_{j \mid i}$, where $p_{j \mid i}$ is the selection probability of the unit $j$ in area $i$. The $p_{j \mid i}$ are defined based on specified size measure $b_{i j}$, which is related to the variable of interest, $y_{i j}$ :

$$
p_{j \mid i}=\frac{b_{i j}}{\sum_{k=1}^{N_{i}} b_{i k}}
$$

The goal is to estimate the small area means $\bar{Y}_{i}=\frac{1}{N_{i}} \sum_{j=1}^{N_{i}} y_{i j}$, which may be approximated by $\mu_{i}=\overline{\mathbf{X}}_{i} \boldsymbol{\beta}+v_{i}$, if population size $N_{i}$ is large, where $\overline{\mathbf{X}}_{i}^{T}=$ $\frac{1}{N_{i}} \sum_{j=1}^{N_{i}} \mathbf{x}_{i j}$ is the known population mean of auxiliary variables in area $i$.

As mentioned in Section 1.1.1, if the sampling design is non-informative, the empirical best linear predictor (EBLUP) estimator of the target parameter $\mu_{i}$ can be obtained as follows (Henderson, 1975):

$$
\begin{aligned}
\hat{\mu}_{i}{ }^{H} & =\overline{\mathbf{X}}_{i}^{T} \hat{\boldsymbol{\beta}}+\hat{v}_{i} \\
& =\hat{\gamma}_{i} \bar{y}_{i}+\left(\overline{\mathbf{X}}_{i}-\hat{\gamma}_{i} \overline{\mathbf{x}}_{i}\right)^{T} \hat{\boldsymbol{\beta}}, \quad i=1, \cdots, m,
\end{aligned}
$$

where $\hat{\gamma}_{i}=\frac{\hat{\sigma}_{v}^{2}}{\hat{\sigma}_{v}^{2}+\hat{\sigma}_{e}^{2} / n_{i}}, \bar{y}_{i}=\frac{1}{n_{i}} \sum_{j=1}^{n_{i}} y_{i j}$ and $\overline{\mathbf{x}}_{i}=\frac{1}{n_{i}} \sum_{j=1}^{n_{i}} \mathbf{x}_{i j}$ are the unweighted sample means of variable of interest $y$ and the covariates $\mathbf{x}$, respectively, and $\hat{\sigma}_{e}$ and $\hat{\sigma}_{v}$ are obtained by the method of fitting constants (Henderson, 1953) or restricted maximum likelihood (REML). Further,

$$
\hat{\boldsymbol{\beta}}=\left\{\sum_{i=1}^{m} \sum_{j=1}^{n_{i}} \mathbf{x}_{i j}\left(\mathbf{x}_{i j}-\hat{\gamma}_{i} \overline{\mathbf{x}}_{i}\right)^{T}\right\}^{-1}\left\{\sum_{i=1}^{m} \sum_{j=1}^{n_{i}}\left(\mathbf{x}_{i j}-\hat{\gamma}_{i} \overline{\mathbf{x}}_{i}\right) y_{i j}\right\}
$$

However, under informative sampling, (2.2) can lead to biased estimates of $\mu_{i}$. Pfeffermann and Sverchkov (2007) studied estimation of small area means under informative sampling, assuming that the model of the sample data and weights 
within selected areas $w_{j \mid i}$ are known. They obtained "optimal" estimators of area means under a particular sample model. Verret, Rao and Hidiroglou (2015) proposed an alternative method using augmented models. They included an additional auxiliary variable in the sample model using a suitable function of the selection probabilities to account for the informative sampling. Since the proposed method in this chapter is referring to methods proposed by Pfeffermann and Sverchkov (2007) and Verret et al.(2015), we briefly describe those two methods in the following subsections. They are also prsented in Section 7.6.3 of Rao and Molina (2015).

\subsubsection{Pfeffermann and Sverchkov's method}

Pfeffermann and Sverchkov (2007) studied estimation of small area means under informative sampling, and proposed a new estimator by correcting the EBLUP estimator for the sample selection bias. They considered the case of sampled and non-sampled areas $(m<M)$. Since the focus of this chapter is on the case where all areas are sampled, we assume that $m=M$. The sample model is assumed to be a nested error model

$$
y_{i j}=\mathbf{x}_{i j}^{T} \boldsymbol{\beta}+v_{i}+e_{i j} \quad i=1, \ldots, m, \quad j \in s_{i},
$$

where $s_{i}$ is the set of sampled units in area $i, v_{i} \stackrel{i i d}{\sim} N\left(0, \sigma_{v}^{2}\right)$ and $e_{i j} \mid j \in s_{i} \stackrel{i i d}{\sim}$ $N\left(0, \sigma_{e}^{2}\right)$. Let $\pi_{j \mid i}$ be the inclusion probability for unit $j$ in area i. It is assumed that the expectation of sampling weights, $w_{j \mid i}=\frac{1}{\pi_{j \mid i}}$, have the following form:

$$
E_{s_{i}}\left(w_{j \mid i} \mid \mathbf{x}_{i j}, y_{i j}, v_{i}, I_{i}=1\right)=k_{i} \exp \left(\mathbf{x}_{i j}^{T} \mathbf{a}+b y_{i j}\right)
$$


where $\mathbf{a}$ and $b$ are fixed unknown constants, and $k_{i}=\frac{N_{i}}{n_{i}}\left[\frac{1}{N_{i}} \sum_{j=1}^{N_{i}} \exp \left(-\mathbf{x}_{i j}^{T} \mathbf{a}-\right.\right.$ $\left.\left.b y_{i j}\right)\right]$. For large $N_{i}, E\left[\frac{1}{N_{i}} \sum_{j=1}^{N_{i}} \exp \left(-\mathbf{x}_{i j}^{T} \mathbf{a}-b y_{i j}\right)\right]$ may be approximated by its population expectation, say a constant $c_{i}$. So, $k_{i}$ in (2.4) is approximately equal to $c_{i} \frac{N_{i}}{n_{i}}$.

Under the above assumption, the best prediction estimator of $\bar{Y}_{i}$ is:

$$
\hat{\bar{Y}}_{i}=\frac{1}{N_{i}}\left\{\left(N_{i}-n_{i}\right)\left(\hat{v}_{i}+\overline{\mathbf{X}}_{i}^{T} \hat{\boldsymbol{\beta}}\right)+n_{i}\left[\bar{y}_{i}+\left(\overline{\mathbf{X}}_{i}-\overline{\mathbf{x}}_{i}\right)^{T} \hat{\boldsymbol{\beta}}\right]+\left(N_{i}-n_{i}\right) \hat{b} \hat{\sigma}_{e}^{2}\right\}
$$

where $\hat{b}$ is obtained by regressing the sampling weights $w_{j \mid i}$ on $k_{i} \exp \left(\mathbf{x}_{i j}^{T} \mathbf{a}+b y_{i j}\right)$, $\hat{v}_{i}=\hat{\gamma}_{i}\left(y_{i}-\bar{X}_{i}^{T} \hat{\boldsymbol{\beta}}\right)$ with $\hat{\gamma}_{i}=\hat{\sigma}_{v}^{2} /\left(\hat{\sigma}_{v}^{2}+n_{i}^{-1} \hat{\sigma}_{e}^{2}\right)$. Estimators $\hat{\boldsymbol{\beta}}, \hat{\sigma}_{v}^{2}$ and $\hat{\sigma}_{e}^{2}$ are obtained by fitting model (2.3) on $y_{i j}$.

It was shown that estimator (2.5) leads to less bias compared to the ordinary EBLUP (i.e., ignoring the informativeness of the design), and it is more efficient.

\subsubsection{Verret, Rao and Hidiroglou's method}

Assuming that all areas are sampled $(m=M)$, Verret, Rao and Hidiroglou (2015) considered the following augmented sample model:

$$
y_{i j}=\mathbf{x}_{i j}^{T} \boldsymbol{\beta}+g\left(p_{j \mid i}\right) \delta+\tilde{v}_{i}+\tilde{e}_{i j}, \quad j=1, \ldots, n_{i} \quad i=1, \ldots, m
$$

where $\tilde{v}_{i} \stackrel{i i d}{\sim} N\left(0, \sigma_{v}^{2}\right)$ is independent of $\tilde{e}_{i j} \sim N\left(0, \sigma_{e}^{2}\right)$, and $g(\cdot)$ is a specific function. The $g\left(p_{j \mid i}\right)$ in (2.6) is the augmenting variable added to the model with the purpose of adjusting the bias due to the informative sampling. Verret et al. (2015) tested different choices of $g\left(p_{j \mid i}\right)$, such as $p_{j \mid i}, \log \left(p_{j \mid i}\right)$ and $w_{j \mid i}$. They mentioned 
that for the selection of $g(\cdot)$ function, residuals of (2.6) can be plotted against $g\left(p_{j \mid i}\right)$.

The EBLUP estimator of the area mean $\bar{Y}_{i}$ under the augmented model (2.6) is given by

$$
\hat{\mu}_{i}=\hat{\gamma}_{i} \bar{y}_{i}+\left(\overline{\mathbf{X}}_{i}-\hat{\gamma}_{i} \overline{\mathbf{x}}_{i}\right)^{T} \hat{\boldsymbol{\beta}}+\left(\bar{G}_{i}-\hat{\gamma}_{i} \bar{g}_{i}\right) \hat{\delta}
$$

where $\bar{G}_{i}$ is the mean of population values of $g\left(p_{j \mid i}\right), \hat{\gamma}_{i}=\frac{\hat{\sigma}_{v}^{2}}{\hat{\sigma}_{v}^{2}+\hat{\sigma}_{e}^{2} / n_{i}}$ and $\hat{\boldsymbol{\beta}}$ and $\hat{\delta}$ are estimated by weighted least squares (WLS).

The method assumes the knowledge of $\bar{G}_{i}$. However, the mean of population values $\bar{G}_{i}$ can be obtained for certain forms of $g\left(p_{j \mid i}\right)$, for example if $g\left(p_{j \mid i}\right)=p_{j \mid i}$, then $\bar{G}_{i}=1$.

Verret et al. (2015) compared the performance of estimator (2.7) with Pfeffermann and Sverchkov's method using the same size measures. They showed that (2.5) can be expressed as an EPLUP estimator of the area mean, $\bar{Y}_{i}$, with the addition of a term for correcting bias due to informative sampling. Their simulation results showed that (2.7) performs well in terms of bias and and significantly better in terms of MSE relative to Pfeffermann and Sverchkov's estimator, if the augmented variable is chosen properly.

\section{$2.3 \quad$ P-spline augmentation}

The idea is to include a P-spline term of selection probabilities in the model to account for the informativeness of the sampling design. We begin by describing penalized splines regression outside of the small area estimation context. 


\subsubsection{Penalized spline regression}

In general, the goal of regression analysis is to model the dependence of the mean of the variable of interest $\mathbf{y}=\left(y_{1}, \cdots, y_{n}\right)^{T}$ on a set of covariate variables $\mathbf{X}=$ $\left(\mathbf{x}_{1}, \cdots, \mathbf{x}_{n}\right)^{T}$ where $\mathbf{x}_{i}=\left(x_{i 1}, x_{i 2}, \cdots, x_{i p}\right)$. More precisely, the interest is to find a function $\mathcal{G}(\cdot)$ such that:

$$
E\left(\mathbf{y} \mid \mathbf{x}_{1}, \ldots, \mathbf{x}_{p}\right)=\mathcal{G}\left(\mathbf{x}_{1}, \ldots, \mathbf{x}_{p}\right)
$$

The function $\mathcal{G}(\cdot)$ can have a certain shape, such as linear or quadratic, that can be estimated parametrically. For example, it can be modeled as a linear combination of covariates, like $\mathcal{G}\left(\mathbf{x}_{1}, \ldots, \mathbf{x}_{p}\right)=\beta_{0}+\sum_{i} \beta_{i} \mathbf{x}_{i}$. These models are easy to estimate and to interpret, however the linear assumption does not hold often. Alternatively, the function of $\mathcal{G}(\cdot)$ can be modeled using some unspecified smooth function of covariates. The functional dependence between the response variable and covariates does not assume any specific parametric assumptions. Splines are a popular family of smoothers.

A spline is a piece-wise polynomial with pieces defined by a sequence of knots, $q_{1}<q_{2}<\ldots<q_{k}$, such that the pieces join smoothly at the knots (Rodriguez, 2011). For simplicity, we assume that one covariate is available $(p=1)$. The function $\mathcal{G}(\cdot)$ is defined by

$$
\mathcal{G}(x)=\beta_{0}+\beta_{1} x+\ldots+\beta_{h} x^{h}+\sum_{k=1}^{K} \gamma_{k}\left(x-q_{k}\right)_{+}^{h}
$$

where $\left(x-q_{k}\right)_{+}=\max \left\{0,\left(x-q_{k}\right)\right\}$ and " $h$ " is the degree of the polynomial. 
We illustrate this with an example. Consider the function $\mathcal{G}(x)=3+\sin (2 \pi x)$ for $200 x$ values which are equally spaced on $[0,1]$ and independent of $\epsilon_{i} \sim$ $N(0,0.4)$ for $i=1, \ldots, 200$. The following figure shows the estimated curves with the quadratic truncated polynomial basis function using 10 knots.

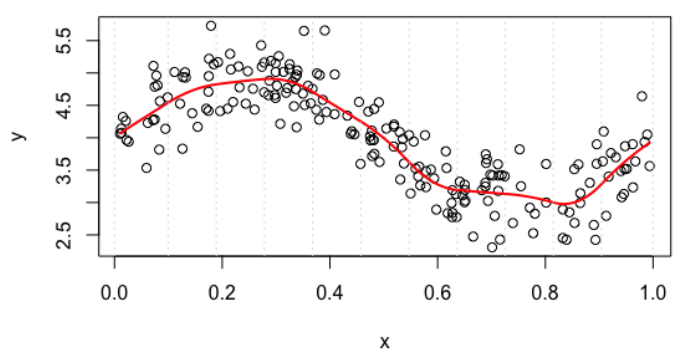

Figure 2.1: Example: piecewise quadratic polynomial with 10 knots

The red line in Figure 2.1 is the estimated curve and the broken vertical lines indicate the position of knots.

The spline function depends on the basis as well as the number of knots. As Ruppert (2002) suggested, the knots are equally spaced and the number of knots are usually set to $\min \{n / 4,40\}$. The model (2.8) could be over-parametrized. To avoid over-parametrization, a constraint (or penalty) is put on the spline parameter $\gamma=\left(\gamma_{1}, \cdots, \gamma_{k}\right)^{T}$,

$$
\sum_{i=1}^{n}\left\{y_{i}-\mathcal{G}\left(x_{i} ; \boldsymbol{\beta}, \boldsymbol{\gamma}\right)\right\}^{2}+\lambda_{\gamma}^{2} \boldsymbol{\gamma}^{T} \boldsymbol{\gamma}
$$

where $\lambda_{\gamma}$ is the fixed penalty parameter. Estimators of $\boldsymbol{\beta}$ and $\boldsymbol{\gamma}$ depend on $\lambda_{\gamma}$. In particular, smaller values of $\lambda_{\gamma}$ result in an "over-fitted" estimate. One way for finding $\lambda_{\gamma}$ is to use cross-validation or similar methods. Another approach is to use a mixed model formulation and treat $\gamma$ as a random effect vector with mean 
$\mathbf{0}$ and covariance $\sigma_{\gamma}^{2} \mathbf{I}$, as suggested by Ruppert et al. (2003). The resulting best linear unbiased estimator (BLUE) of $\boldsymbol{\beta}$ and BLUP of $\boldsymbol{\gamma}$ are the equivalent to the corresponding penalized least squares estimator. We follow the second approach and treat $\gamma$ as a random effect in a linear mixed model. This would be in line with small area estimation as the relationship between variable of interest and auxiliary information is usually expressed in the form of a linear mixed model with random effect for small areas.

\subsubsection{P-spline mixed model}

As mentioned before, both small area models and P-spline models can be expressed as a linear mixed model, and as a result can be combined together. Opsomer et al. (2008) extended the P-spline to small area estimation and explained in detail how to combine both models.

\subsection{Proposed method}

Going back to the suggested method, the goal is to replace the $g\left(p_{j \mid i}\right)$ in $(2.6)$ with a function $\mathcal{G}\left(p_{j \mid i}\right)$ where $\mathcal{G}$ is an unknown function defined using P-spline (similar to (2.8)). Assuming that $p$ covariates are available, the model is given by:

$$
y_{i j}=\mathbf{x}_{i j}^{T} \boldsymbol{\beta}+\mathcal{G}\left(p_{j \mid i}\right)+\tilde{v}_{i}+\tilde{e}_{i j} \quad j=1, \ldots, n_{i} \quad i=1, \ldots, m
$$

where $\mathcal{G}\left(p_{j \mid i}\right)$ is defined as follows:

$$
\mathcal{G}\left(p_{j \mid i}\right)=\alpha_{0}+\alpha_{1} p_{j \mid i}+\ldots+\alpha_{h} p_{j \mid i}^{h}+\sum_{k=1}^{K} \gamma_{k}\left(p_{j \mid i}-q_{k}\right)_{+}^{h}
$$


where

$h$ is the degree of the spline,

$\left\{q_{1}, \ldots, q_{k}\right\}$ is the set of the fixed knots,

$\boldsymbol{\beta}=\left(\beta_{1}, \beta_{2}, \cdots, \beta_{p}\right)^{T}$ is the coefficient term corresponding to $\mathbf{x}_{i j}=$ $\left(x_{i j 1}, \cdots, x_{i j p}\right)^{T}$,

$\boldsymbol{\alpha}$ is the coefficient vector of the parameter $\boldsymbol{\alpha}=\left(\alpha_{0}, \alpha_{1}, \ldots, \alpha_{h}\right)^{T}$ in $(2.10)$,

$\gamma$ is the coefficient vector of the spline portion $\gamma=\left(\gamma_{1}, \ldots, \gamma_{k}\right)^{T}$,

and $\left(p_{j \mid i}-q_{k}\right)_{+}^{h}$ denotes $\left(p_{j \mid i}-q_{k}\right)^{h} \mathbf{I}_{\left(p_{j \mid i}\right)>0}$.

Substituting (2.10) in $(2.9)$ for $\mathcal{G}\left(p_{j \mid i}\right)$, and writing the equation in the matrix form we have (using Opsomer et al. (2008) notation):

$$
\mathbf{y}=\mathbf{X} \boldsymbol{\beta}+\mathbf{P} \boldsymbol{\alpha}+\mathbf{D} \boldsymbol{\gamma}+\mathbf{Z} \mathbf{v}+\mathbf{e}
$$

where $\mathbf{y}=\operatorname{col}_{1 \leq i \leq m}\left(\mathbf{y}_{i}\right)$ with $\mathbf{y}_{i}=\left(y_{i 1}, \cdots, y_{i n_{i}}\right)^{T}, \mathbf{X}=\operatorname{col}_{1 \leq i \leq m}\left(\mathbf{X}_{i}\right)$, where $\mathbf{X}_{i}$ is a $n_{i} \times p$ matrix with rows equal to $\mathbf{x}_{i j}$ for $j=1, \cdots n$, e $=\operatorname{col}_{1 \leq i \leq m}\left(\mathbf{e}_{i}\right)$ with $\mathbf{e}_{i}=\left(e_{i 1}, \cdots, e_{i n_{i}}\right)^{T}$, and $\mathbf{v}=\left(v_{1}, \cdots, v_{m}\right)^{T}$. The matrix $\mathbf{Z}$ is defined as $\mathbf{Z}=\operatorname{diag}_{(1 \leq i \leq m)}\left(\mathbf{Z}_{i}\right)$, where $\mathbf{Z}_{i}=\mathbf{1}_{n_{i}}$. $\mathbf{P}$ and $\mathbf{D}$ represent the design matrices of fixed and random effects of the P-spline term respectively:

$$
\mathbf{P}=\left[\begin{array}{cccc}
1 & p_{1 \mid 1} & \cdots & p_{1 \mid 1}^{h} \\
\vdots & \vdots & & \vdots \\
1 & p_{n_{m} \mid m} & \cdots & p_{n_{m} \mid m}{ }^{h}
\end{array}\right]_{n \times h+1} \text { and } \mathbf{D}=\left[\begin{array}{ccc}
\left(p_{1 \mid 1}-q_{1}\right)_{+}^{h} & \cdots & \left(p_{1 \mid 1}-q_{k}\right)_{+}^{h} \\
\vdots & \vdots & \\
& & \\
\left(p_{n_{m} \mid m}-q_{1}\right)_{+}^{h} & \cdots & \left(p_{n_{m} \mid m}-q_{k}\right)_{+}^{h}
\end{array}\right]_{n \times k} .
$$


Both $\mathbf{X}$ and $\mathbf{P}$ are fixed effects in the model and can be combined together as matrix A given by

$$
\mathbf{A}=\left[\begin{array}{ccc|cccc}
x_{11} & \cdots & x_{111} & 1 & p_{1 \mid 1} & \cdots & p_{1 \mid 1}{ }^{h} \\
\vdots & & \vdots & \vdots & \vdots & & \vdots \\
x_{m n_{m}} & \cdots & x_{m n_{n} p} & 1 & p_{n_{m} \mid m} & \cdots & p_{n_{m} \mid m}{ }^{h}
\end{array}\right]_{n \times(p+h+1)}
$$

Let $\boldsymbol{\beta}^{\star}=\left(\beta_{1}, \cdots, \beta_{p}, \alpha_{0}, \alpha_{1}, \cdots, \alpha_{h}\right)^{T}$, then $(2.11)$ can be written as

$$
\mathbf{y}=\mathbf{A} \boldsymbol{\beta}^{\star}+\mathbf{D} \gamma+\mathbf{Z v}+\mathbf{e}
$$

Assuming that $\boldsymbol{\gamma} \sim\left(\mathbf{0}, \sigma_{k}^{2} \mathbf{I}_{k}\right), \mathbf{v} \sim\left(\mathbf{0}, \sigma_{v}^{2} \mathbf{I}_{m}\right)$ and $\mathbf{e} \sim\left(\mathbf{0}, \sigma_{e}^{2} \mathbf{I}_{n}\right)$, the best linear unbiased predictor (BLUP) of $\boldsymbol{\theta}=\left(\boldsymbol{\beta}^{\star}, \boldsymbol{\gamma}, \mathbf{v}\right)^{T}$ can be found by solving the mixed model equations (Henderson, 1963):

$$
\left[\begin{array}{ccc}
\frac{1}{\sigma_{e}^{2}} \mathbf{A}^{T} \mathbf{A} & \frac{1}{\sigma_{e}^{2}} \mathbf{A}^{T} \mathbf{D} & \frac{1}{\sigma_{e}^{2}} \mathbf{A}^{T} \mathbf{Z} \\
\frac{1}{\sigma_{e}^{2}} \mathbf{D}^{T} \mathbf{A} & \frac{1}{\sigma_{\gamma}^{2}} \mathbf{I}_{k}+\frac{1}{\sigma_{e}^{2}} \mathbf{D}^{T} \mathbf{D} & \frac{1}{\sigma_{e}^{2}} \mathbf{D}^{T} \mathbf{Z} \\
\frac{1}{\sigma_{e}^{2}} \mathbf{Z}^{T} \mathbf{A} & \frac{1}{\sigma_{e}^{2}} \mathbf{Z}^{T} \mathbf{D} & \frac{1}{\sigma_{v}^{2}} \mathbf{I}_{m}+\frac{1}{\sigma_{e}^{2}} \mathbf{Z}^{T} \mathbf{Z}
\end{array}\right]\left[\begin{array}{c}
\boldsymbol{\beta}^{\star} \\
\boldsymbol{\gamma} \\
\mathbf{v}
\end{array}\right]=\left[\begin{array}{c}
\frac{1}{\sigma_{e}^{2}} \mathbf{A}^{T} \mathbf{y} \\
\frac{1}{\sigma_{e}^{2}} \mathbf{D}^{T} \mathbf{y} \\
\frac{1}{\sigma_{e}^{2}} \mathbf{Z}^{T} \mathbf{y}
\end{array}\right]
$$

Let $\mathbf{W}=\left[\begin{array}{lll}W_{11} & W_{12} & W_{13} \\ W_{21} & W_{22} & W_{23} \\ W_{31} & W_{32} & W_{33}\end{array}\right]$ be the inverse of the first matrix in the left side of (2.12). With the above notation, the solution of (2.12) is:

$$
\left[\begin{array}{c}
\tilde{\boldsymbol{\beta}}^{\star} \\
\tilde{\boldsymbol{\gamma}} \\
\tilde{\mathbf{v}}
\end{array}\right]=\left[\begin{array}{c}
\frac{1}{\sigma_{e}^{2}}\left(W_{11} \mathbf{A}^{T}+W_{12} \mathbf{D}^{T}+W_{13} \mathbf{Z}^{T}\right) \mathbf{y} \\
\frac{1}{\sigma_{e}^{2}}\left(W_{21} \mathbf{A}^{T}+W_{22} \mathbf{D}^{T}+W_{23} \mathbf{Z}^{T}\right) \mathbf{y} \\
\frac{1}{\sigma_{e}^{2}}\left(W_{31} \mathbf{A}^{T}+W_{32} \mathbf{D}^{T}+W_{33} \mathbf{Z}^{T}\right) \mathbf{y}
\end{array}\right] .
$$


The parameter $\boldsymbol{\sigma}=\left(\sigma_{e}^{2}, \sigma_{\gamma}^{2}, \sigma_{v}^{2}\right)^{T}$ is estimated iteratively by fixed-point equations

$$
\begin{gathered}
\sigma_{\gamma}^{2}=\sum_{k=1}^{K} \frac{\tilde{\gamma}_{k}^{2}}{k-\left(\operatorname{tr}\left(W_{22}\right) / \sigma_{\gamma}^{2}\right)}, \\
\sigma_{v}^{2}=\sum_{i=1}^{m} \frac{\tilde{v}_{i}^{2}}{m-\left(\operatorname{tr}\left(W_{33}\right) / \sigma_{v}^{2}\right)}, \\
\sigma_{e}^{2}=\sum_{i=1}^{m} \sum_{j=1}^{n_{i}} \frac{\tilde{e}_{i j}^{2}}{n-\left(p+\left(k-\operatorname{tr}\left(W_{22}\right) / \sigma_{\gamma}^{2}\right)\right)+\left(m-\left(\operatorname{tr}\left(W_{33}\right) / \sigma_{v}^{2}\right)\right)},
\end{gathered}
$$

where $\tilde{v}_{i}, \tilde{\gamma}_{k}$ are obtained from (2.13) and $\tilde{e}_{i j}$ is equal to

$$
\tilde{e}_{i j}=y_{i j}-\mathbf{x}_{i j}^{T} \tilde{\boldsymbol{\beta}}^{\star}-\mathbf{D}_{i j}^{T} \tilde{\boldsymbol{\gamma}}-\tilde{v}_{i}
$$

Hence, the small area means $\bar{Y}_{i}$ are computed iteratively using the following steps:

1. Compute the terms on the right side of the equations (2.14) -(2.16) using $\tilde{\gamma}$, $\tilde{\mathbf{v}}$ and $\tilde{\boldsymbol{e}}$ values from $(2.12)$ with starting values $\boldsymbol{\sigma}^{(0)}=\left({\sigma_{e}^{2(0)}}^{\left(\sigma_{\gamma}^{2}(0)\right.},{\sigma_{v}^{2}}^{(0)}\right)^{T}$ to get $\boldsymbol{\sigma}^{(1)}$

2. Using $\boldsymbol{\sigma}^{(1)}$, compute $\boldsymbol{\sigma}^{(2)}$ from (2.14) -(2.16) and repeat this step until convergence to get $\hat{\boldsymbol{\sigma}}=\left(\hat{\sigma}_{e}^{2}, \hat{\sigma}_{\gamma}^{2}, \hat{\sigma}_{v}^{2}\right)^{T}$

3. Substitute $\hat{\boldsymbol{\sigma}}$ for $\boldsymbol{\sigma}$ into (2.13) to obtain the EBLUP of $\boldsymbol{\beta}, \boldsymbol{\gamma}$ and $\mathbf{v}$

4. The EBLUP estimator of small area means are given by

$$
\hat{\mu}_{i}=\frac{1}{N_{i}}\left(\sum_{j \in s_{i}} y_{i j}+\sum_{j \in \bar{s}_{i}} \hat{y}_{i j}\right),
$$


where $\bar{s}_{i}$ represents the non-sampled units and $\hat{y}_{i j}$ is the predictor of $y_{i j}$ for the non-sampled units using the spline approximation:

$$
\hat{y}_{i j}=\mathbf{A}_{i j}^{T} \hat{\boldsymbol{\beta}}^{\star}+\mathbf{D}_{i j}^{T} \hat{\gamma}+\hat{v}_{i}
$$

where $\mathbf{A}_{i j}=\left(x_{i j_{1}}, \cdots, x_{i j_{p}}, 1, p_{j \mid i}, \cdots, p_{j \mid i}^{h}\right)^{T}$ and $\left.\mathbf{D}_{i j}=\left(\left(p_{j \mid i}-q_{1}\right)_{+}^{h}, \cdots, p_{j \mid i}-q_{k}\right)_{+}^{h}\right)^{T}$.

It is assumed that $p_{j \mid i}$ 's for non-sampled units in area $i$ are known.

\subsection{Mean squared error}

In general, the mean squared prediction error (MSE) gives a simple description for performance of the model. The MSE of an estimator $\hat{\theta}$ of parameter $\theta$ is given by

$$
\operatorname{MSE}(\hat{\theta})=E(\hat{\theta}-\theta)^{2} .
$$

where " $E$ " indicates the model expectation. The $M S E(\hat{\theta})$ is also called mean squared prediction error, as $\theta$ is random. When the sampling is not informative, the MSE of the EBLUP estimator of small area mean $\mu_{i}$, under the unit-level model, can be decomposed as (see Rao and Molina (2015), page 179):

$$
\operatorname{MSE}\left(\hat{\mu}_{i}\right)=g_{1}(\hat{\delta})+g_{2}(\hat{\delta})+2 g_{3}(\hat{\delta})
$$


where

$$
\begin{aligned}
& \hat{\delta}=\left(\hat{\sigma}_{e}^{2}, \hat{\sigma}_{v}^{2}\right), \\
& g_{1}(\hat{\delta})=\left(1-\hat{\gamma}_{i}\right) \hat{\sigma}_{v}^{2}, \\
& g_{2}(\hat{\delta})=\left(\overline{\mathbf{X}}_{i}-\hat{\gamma}_{i} \overline{\mathbf{x}}_{i}\right)^{T}\left(\sum_{i=1}^{M} \mathbf{X}_{i}^{T} \hat{\mathbf{V}}_{i}^{-1} \mathbf{X}_{i}\right)^{-1}\left(\overline{\mathbf{X}}_{i}-\hat{\gamma}_{i} \overline{\mathbf{x}}_{i}\right), \\
& g_{3}(\hat{\delta})=\hat{\gamma}_{i}(1-\hat{\gamma})^{2} \hat{\sigma}_{e}^{-4} \hat{\sigma}_{v}^{-2}\left\{\hat{\sigma}_{e}^{4} \operatorname{var}\left(\hat{\sigma}_{v}^{2}\right)-2 \hat{\sigma}_{e}^{2} \hat{\sigma}_{v}^{2} \operatorname{cov}\left(\hat{\sigma}_{v}^{2}, \hat{\sigma}_{e}^{2}\right)+\hat{\sigma}_{v}^{4} \operatorname{var}\left(\hat{\sigma}_{e}^{2}\right)\right\}
\end{aligned}
$$

where $\hat{\gamma}_{i}=\frac{\hat{\sigma}_{v}^{2}}{\hat{\sigma}_{v}^{2}+\hat{\sigma}_{e}^{2} / n_{i}}, \hat{V}_{i}=\hat{\sigma}_{e}^{2} \mathbf{I}_{n_{i}}+\hat{\sigma}_{v}^{2} \mathbf{1}_{n_{i}} \mathbf{1}_{n_{i}}^{T}$ and $\mathbf{X}_{i}^{T}=\left(\mathbf{x}_{i 1}, \ldots, \mathbf{x}_{i n_{i}}\right)$.

For informative sampling, Pfeffermann and Sverchkov (2007) proposed a bootstrap method to estimate MSE, while Verret et al. (2015) updated formulas in (2.18) and applied it to the augmented model in (2.7).

For the proposed P-spline method, we will follow the approach suggested by Rao, Sinha and Dumitrescu (2014). They proposed a conditional bootstrap method for estimating the MSE. They noted that the randomness of the spline term " $\gamma$ " is a device used to model curvature and the inference should be conditioned on $\hat{\gamma}$, as stated in Ruppert et al. (2003). Following the method described in Rao et al. (2014), the inference is conditional on the $\hat{\gamma}$ which is defined based on the $p_{j \mid i}$. We generate $v_{i}^{\bullet} \sim N\left(0, \hat{\sigma}_{v}^{2}\right)$ and $e_{i j}^{\bullet} \sim N\left(0, \hat{\sigma}_{e}^{2}\right)$ and obtain the bootstrap responses as follows:

$$
y_{i j}^{\bullet}=\mathbf{A}_{i j}^{T} \hat{\boldsymbol{\beta}}^{\star}+\mathbf{D}_{i j}^{T} \hat{\gamma}+v_{i}^{\bullet}+e_{i j}^{\bullet} \quad i=1, \ldots, m, \quad j=1, \ldots, N_{i}
$$

where $\hat{\boldsymbol{\beta}}^{\star}$ and $\hat{\boldsymbol{\gamma}}$ are the EBLUP estimates of $\boldsymbol{\beta}^{\star}=\left(\beta_{1}, \cdots, \beta_{p}, \alpha_{0}, \alpha_{1}, \cdots, \alpha_{h}\right)^{T}$ and $\gamma=\left(\gamma_{1}, \ldots, \gamma_{k}\right)^{T}$, respectively. 
Using the corresponding bootstrap sample data $\left(y_{i j}^{\bullet}, \mathbf{A}_{i j}, \mathbf{D}_{i j}\right), i=1, \cdots m$, $j \in s_{i}$, the bootstrap estimates $\hat{\boldsymbol{\beta}}^{\bullet}, \hat{\boldsymbol{\gamma}}^{\bullet}$ and $\hat{v}_{i}^{\bullet}$ are computed and the bootstrap predicted values $\hat{y}_{i j}^{\bullet}$ for $j \in \bar{s}_{i}$, where $\bar{s}_{i}$ is the set of non-sampled units in area $i$, can be obtained as

$$
\hat{y}_{i j}^{\bullet}=\mathbf{A}_{i j}^{T} \hat{\boldsymbol{\beta}}^{\bullet}+\mathbf{D}_{i j}^{T} \hat{\gamma}^{\bullet}+\hat{v}_{i}^{\bullet} .
$$

The bootstrap EBLUP of the $\bar{Y}_{i}$ is then given by

$$
\hat{\mu}_{i}^{\bullet}=\frac{1}{N_{i}}\left(\sum_{j \in s_{i}} y_{i j}^{\bullet}+\sum_{j \in \bar{s}_{i}} \hat{y}_{i j}^{\bullet}\right) .
$$

Repeating the above procedure B times, the conditional bootstrap estimator of MSE of the EBLUP of $\bar{Y}_{i}$ can be approximated by

$$
\hat{M}_{b o o t}\left(\hat{\mu}_{i}\right)=\frac{1}{B} \sum_{b=1}^{B}\left(\hat{\mu}_{i}^{\bullet}(b)-\bar{Y}_{i}^{\bullet}(b)\right)^{2},
$$

where $\hat{\mu}_{i}^{\bullet}(b)$ and $\bar{Y}_{i}^{\bullet}(b)$ are the values of $\hat{\mu}_{i}^{\bullet}$ and $\bar{Y}_{i}^{\bullet}$ for the $b^{\text {th }}$ bootstrap replicate, and $\bar{Y}_{i}^{\bullet}=\frac{1}{N_{i}} \sum_{j=1}^{N_{i}} y_{i j}^{\bullet}$.

\subsection{Simulation study}

In this section, we present results of a limited simulation study on the performance of the proposed method. The area means are estimated and the bias and the MSE of the estimates are compared with the method proposed by Verret et al. (2015), described in section 2.2.2 and the ordinary EBLUP. 
Similar to Verret et al. (2015), we considered a design-model approach for generating data. The population data $y_{i j}$ are generated from the model

$$
y_{i j}=\beta_{0}+\beta_{1} x_{i j}+v_{i}+e_{i j} \quad i=1, \ldots, 99, \quad j=1, \ldots, N_{i},
$$

where $\beta_{0}=1, \beta_{1}=1, v_{i} \sim N(0,0.5)$ and $e_{i j} \sim N(0,2)$. The $x_{i j}$ values are generated from gamma with mean 10 and variance 50; they are held fixed throughout the simulation. We considered two settings for the number of units in each area to study the impact of $N_{i}$ on the performance of the p-spline method:

(i) Equal area sizes:

$$
N_{i}=100 \text { for } i=1, \ldots, 99
$$

(ii) Unequal area sizes:

$$
\begin{aligned}
& N_{1}=N_{2}=\cdots=N_{33}=50, \\
& N_{34}=N_{35}=\cdots=N_{66}=100, \\
& N_{67}=N_{68}=\cdots=N_{99}=150 .
\end{aligned}
$$

Different sample sizes are also considered within areas by fixing $n_{1}=n_{2}=$ $\cdots=n_{33}=5, n_{34}=n_{35}=\cdots=n_{66}=7$ and $n_{67}=n_{68}=\cdots=n_{99}=9$. The total sample size is $n=\sum_{i=1}^{m} n_{i}=693$.

The samples are selected within each area with probabilities proportional to size measures $b_{i j}$, using Rao-Sampford method of sampling without replacement with unequal probabilities (Rao (1965) and Sampford (1967)). The Rao-Sampford method ensures that inclusion probabilities $\pi_{j \mid i}$ are proportional to $b_{i j}$, with $\pi_{j \mid i}=$ $\frac{n_{i} b_{i j}}{B_{i}}$, where $B_{i}$ is the total of the $b_{i j}$ in area $i$. 
Similar to Verret et al. (2015), the size measures $b_{i j}$ are generated as follows

$$
b_{i j}=\exp \left[\left\{-\left(v_{i}+e_{i j}\right) / \sigma_{e}+\delta_{i j} / 5\right\} / 3\right]
$$

where $\delta_{i j} \sim N(0,1)$.

A total of $R=1000$ samples were generated using (2.20). For each simulated sample, small area means are computed using (2.17). For the P-spline method, following the recommendations by Ruppert et al. (2003) (section 5.5), we set the number of knots equal to 30 .

Two performance measures are considered: average absolute bias $(\overline{A B})$ and average root MSE $(\overline{R M S E})$. The average absolute bias of the EBLUP estimator of the area mean is defined as

$$
\overline{A B}=\frac{1}{M} \sum_{i=1}^{M} A B_{i},
$$

with

$$
A B_{i}=\left|\frac{1}{R} \sum_{r=1}^{R}\left(\hat{\bar{Y}}_{i}^{(r)}-\bar{Y}_{i}^{(r)}\right)\right|
$$

where $\hat{\bar{Y}}_{i}^{(r)}$ is the EBLUP estimate of the area means computed by (2.17), and $\bar{Y}_{i}^{(r)}$ is the simulated true area mean for the $r^{t h}$ simulated sample. The average root MSE is calculated as:

$$
\overline{R M S E}=\frac{1}{M} \sum_{i=1}^{M} \sqrt{\frac{1}{R} \sum_{r=1}^{R}\left(\hat{\bar{Y}}_{i}^{(r)}-\bar{Y}_{i}^{(r)}\right)^{2}} .
$$

Table 2.1 presents $\overline{A B}$ and $\overline{R M S E}$ for ordinary EBLUP, the augmented model with $g\left(p_{j \mid i}\right)=p_{j \mid i}, \log \left(p_{j \mid i}\right)$ and $n_{i} w_{j \mid i}$, as well as the P-spline method. 
Table 2.1: Average absolute bias and average RMSE of the estimators

\begin{tabular}{||ccccccc||}
\hline$N_{i}$ & Performance measure & Ordinary & $p_{j \mid i}$ & $\log \left(p_{j \mid i}\right)$ & $n_{i} w_{j \mid i}$ & p-spline \\
\hline \hline Equal & $\overline{A B}$ & 0.459 & 0.041 & 0.002 & 0.003 & 0.002 \\
& $\overline{R M S E}$ & 0.626 & 0.153 & 0.108 & 0.155 & 0.107 \\
\hline Unequal & $\overline{A B}$ & 0.457 & 0.150 & 0.005 & 0.178 & 0.005 \\
& $\overline{R M S E}$ & 0.625 & 0.297 & 0.108 & 0.299 & 0.108 \\
& & & & & & \\
\hline
\end{tabular}

As expected, ignoring the informative sampling scheme results in large prediction bias. In particular, the $\overline{A B}$ of the ordinary EBLUP is relatively large compared to augmented models $\left(p_{j \mid i}, \log \left(p_{j \mid i}\right)\right.$ and $\left.n_{i} w_{j \mid i}\right)$ as well as the P-spline method. The predictors of the P-spline method are literally unbiased. The $\overline{R M S E}$ of the ordinary EBLUP has the largest value (0.62). Among other methods, pspline and $\log \left(p_{j \mid i}\right)$ have the smallest $\overline{R M S E}$.

Comparing the results of $\log \left(p_{j \mid i}\right)$ and the p-spline method shows that the performance of the p-spline method is as good as the choice $\log \left(p_{j \mid i}\right)$ in the augmented model, which is the best choice among others, under the chosen size measures.

Table 2.1 shows that unequal $N_{i}$ has an impact on the performance of the augmented model for some choices $\left(p_{j \mid i}\right.$ and $\left.n_{i} w_{j \mid i}\right)$ and increases $\overline{A B}$ significantly. However, it does not affect the performance of the P-spline approach.

We also computed the bootstrap MSE estimator using $B=200$ bootstrap samples for each of the $R=1000$ simulation runs. Let $\hat{M}_{\text {boot }}^{(r)}\left(\hat{\mu}_{i}\right)$ denote the bootstrap MSE estimate of $\hat{\mu}_{i}$ obtained from the $r^{\text {th }}$ simulated sample using (2.19). Let $M S E\left(\hat{\mu}_{i}\right)$ be the approximated true MSE obtained using $\mathrm{T}=10000$ simulated 
data, i.e.,

$$
\widehat{M S E}\left(\hat{\mu}_{i}\right)=\frac{1}{T} \sum_{t=1}^{T}\left(\hat{\mu}_{i}^{(t)}-\bar{Y}_{i}^{(t)}\right)^{2}
$$

where $\hat{\mu}_{i}^{(t)}$ and $\bar{Y}_{i}^{(t)}$ are $\hat{\mu}_{i}$ and $\bar{Y}_{i}$ based on the $t^{\text {th }}$ simulated sample and population, respectively. Using the bootstrap estimates $\hat{M}_{\text {boot }}^{(r)}\left(\hat{\mu}_{i}\right)$, we estimate the mean of the MSE estimator by

$$
\widehat{E}\left\{M S E\left(\hat{\mu}_{i}\right)\right\}=\frac{1}{R} \sum_{r=1}^{R} \hat{M}_{\text {boot }}^{(r)}\left(\hat{\mu}_{i}\right) .
$$

Considering all sampled areas, we consequently estimate the average absolute relative bias $(\overline{A R B})$ of $M S E\left(\hat{\mu}_{i}\right)$ as

$$
\overline{A R B}=\frac{1}{m} \sum_{i=1}^{m}\left|\frac{\hat{E}\left\{M S E\left(\hat{\mu}_{i}\right)\right\}}{\widehat{M S E}\left(\hat{\mu}_{i}\right)}-1\right| .
$$

Table 2.2 reports the $A R B(\%)$ of the MSE estimator, averaged over all areas. For the MSE of the ordinary EBLUP and parametric augmented models, equation (2.18) and its modified version given by Verret et al. (2015) are used, respectively.

Table 2.2: Percent average absolute relative bias of the bootstrap MSE

\begin{tabular}{||ccc||}
\hline \multicolumn{3}{|c|}{$\overline{A R B}(\%)$} \\
\hline Method & Equal $N_{i}$ & Unequal $N_{i}$ \\
\hline \hline Ordinary & 59.4 & 58.6 \\
P-spline & 3.6 & 3.5 \\
$p_{j \mid i}$ & 7.3 & 8.7 \\
$\log \left(p_{j \mid i}\right)$ & 4.3 & 4.3 \\
$n_{i} w_{j \mid i}$ & 8.1 & 9.3 \\
\hline
\end{tabular}


Table 2.2 shows that the bootstrap MSE of the proposed estimator performs well in terms of the average absolute relative bias. The ordinary EBLUP has the largest $\overline{A R B}(\%)$ ( $59 \%$ ) value compared to $\log \left(p_{j \mid i}\right)$ and $p_{j \mid i}$. The P-spline and the choice of $\log \left(p_{j \mid i}\right)$ have the smallest $\overline{A R B}(\%)$. The size of areas $N_{i}$ (equal or not equal areas) does not have much impact on the MSE.

\subsection{Summary}

In this chapter, we studied unit level models under one-stage informative sampling, and proposed a semi-parametric approach to compensate the bias due to informativeness of the sampling design. We included a P-spline term of the selection probabilities in the model. The performance of the proposed estimator was compared to an alternative method proposed by Verret et al.(2015). A conditional bootstrap method for estimating the mean squared error was also proposed.

Results from a simulation study showed that ignoring the informativeness of the sampling design affects the bias and MSE of the EBLUP estimators. The proposed estimator performs well in terms of bias and MSE, and the bias and MSE of the P-spline estimator are comparable to the best choice of the parametric augmented model proposed by Verret et al. (2015). The advantage of the P-spline method is due to its non-parametric nature; the method can be used for any design. Using the P-spline approach, we do not need to go through the process of selecting the augmented function $g(\cdot)$ and test the model with different augmented functions, as described in Verret et al. (2015). 


\section{Chapter 3}

\section{Semi-parametric small area estimation under two-stage informative sampling}

In continuation of the previous chapter, we now consider the case where not all areas are sampled $(m<M)$, and extend the proposed method to two-stage sampling. In this situation, the sampling is done in two stages: first a sample of areas is drawn and second a sample of units is drawn from the selected areas.

As discussed in the previous chapter, if the sampling design is informative at any stage, the population models may not hold for the sample and standard model-based estimators may be biased. Hence, the selection effects should be considered in the inference process. For the two-stage informative sampling, selection effects of both stages of sampling (i.e, areas and units) should be accounted at the inference step. Two-stage informative sampling is studied by Pfeffermann and 
Sverchkov (2007). This chapter proposes a novel P-spline augmentation approach by extending our work in Chapter 2.

\subsection{Two-stage informative sampling}

Two-stage sampling designs are frequently used for social and health surveys. The main advantage of a two-stage sampling is that it is more flexible and less costly than a one-stage sampling design. The sampling mechanism involves two steps: first a random sample, $s$, of $m$ areas is selected, then a sample of $n_{i}$ units is drawn in each selected areas. Let $I_{i}$ denotes the sample indicator variable for the first stage, where $I_{i}=1$ if area $i \in s$, and $I_{i}=0$ otherwise.

For two-stage informative sampling, there would be three possible cases, depending on the sampling design of each stage.

- Case I: both stages are informative

- Case II: stage 1 is informative, and stage 2 is not informative

- Case III: stage 1 is not informative and stage 2 is informative

\subsection{Proposed method}

As mentioned in chapter 2, when the sampling design is informative, selection probabilities should be accounted for in the inference. With regard to the proposed method (P-spline technique), for a two-stage sampling design we have two P-spline

terms: $\mathcal{G}_{1}\left(p_{i}\right)$ and $\mathcal{G}_{2}\left(p_{j \mid i}\right)$. The term $\mathcal{G}_{1}\left(p_{i}\right)$ accounts for the informativeness of the design in the first stage and $\mathcal{G}_{2}\left(p_{j \mid i}\right)$ adjusts the bias due to the informative sampling at the second stage. 


\subsubsection{Case I: both stages are informative}

Consider a finite population $U$ of $M$ areas. The primary sampling units (PSU) are areas, which are selected with selection probabilities of $p_{i}$. The selection probabilities are defined based on specified size measures $b_{i}$ 's, which are related to the variable of interest $y_{i j}$. Let $N_{i}, i=1, \cdots, M$, be the number of secondary sampling units (SSU) in the $i^{\text {th }}$ area (i.e., PSU). In each selected area, a sample of $n_{i}, i=1, \cdots, m$ units are drawn with selection probabilities of $p_{j \mid i}$, based on specified size measures $b_{i j}$ 's, which are also related to the variable of interest, $y_{i j}$. Let $\mathbf{x}_{i j}$ be a p-dimensional covariate vector associated with $y_{i j}$. The sample model can be expressed as follows:

$$
y_{i j}=\mathbf{x}_{i j}^{T} \boldsymbol{\beta}+\mathcal{G}_{1}\left(p_{i}\right)+\mathcal{G}_{2}\left(p_{j \mid i}\right)+v_{i}+e_{i j}, \quad i=1, \cdots, m, \quad j=1, \cdots, n_{i},
$$

where

$\boldsymbol{\beta}=\left(\beta_{0}, \beta_{1}, \beta_{2}, \cdots, \beta_{p}\right)^{T}$ is the coefficient term corresponding to $\mathbf{x}_{i j}=$ $\left(1, x_{i j 1}, \cdots, x_{i j p}\right)^{T}$,

$v_{i}$ is an area-level random effect,

$$
\begin{aligned}
& \mathcal{G}_{1}\left(p_{i}\right)=\lambda_{1} p_{i}+\lambda_{2} p_{i}^{2}+\cdots+\lambda_{l} p_{i}^{l}+\sum_{t=1}^{T} \tau_{t}\left(p_{i}-t_{t}\right)_{+}^{l}, \\
& \mathcal{G}_{2}\left(p_{j \mid i}\right)=\alpha_{1} p_{j \mid i}+\alpha_{1} p_{j \mid i}^{2}+\cdots+\alpha_{h} p_{j \mid i}^{h}+\sum_{k=1}^{K} \gamma_{k}\left(p_{j \mid i}-q_{k}\right)_{+}^{h},
\end{aligned}
$$

$h$ is the degree of spline for $p_{j \mid i}$,

$l$ is the degree of spline for $p_{i}$,

$K$ is the total number of knots for $p_{j \mid i}$, 
$T$ is the total number of knots for $p_{i}$,

$q_{1}, \ldots, q_{K}$ is the set of the fixed knots for $p_{j \mid i}$

$t_{1}, \ldots, t_{T}$ is the set of the fixed knots for $p_{i}$

$\alpha_{1}, \ldots, \alpha_{h}$ is the coefficient vector of the parametric portion of the $p_{j \mid i}$ model,

$\lambda_{1}, \ldots, \lambda_{l}$ is the coefficient vector of the parametric portion of the $p_{i}$ model,

$\gamma_{1}, \ldots, \gamma_{k}$ is the coefficient vector of the spline portion of the $p_{j \mid i}$ model,

$\tau_{1}, \ldots, \tau_{t}$ is the coefficient vector of the spline portion for the $p_{i}$ model.

Writing (3.1) in matrix form we have

$$
\mathbf{y}=\mathbf{X} \boldsymbol{\beta}+\mathbf{P} \boldsymbol{\alpha}+\mathrm{D} \boldsymbol{\gamma}+\mathbf{M} \boldsymbol{\lambda}+\mathbf{B} \boldsymbol{\tau}+\mathrm{Zv}+\mathbf{e}
$$

where $\mathbf{y}=\operatorname{col}_{1 \leq i \leq m}\left(\mathbf{y}_{i}\right)$ with $\mathbf{y}_{i}=\left(y_{i 1}, \cdots, y_{i n_{i}}\right)^{T}, \mathbf{X}=\operatorname{col}_{1 \leq i \leq m}\left(\mathbf{X}_{i}\right)$ where $\mathbf{X}_{i}$ is a $n_{i} \times p$ matrix with rows equal to $\mathbf{x}_{i j}$ for $j=1, \cdots n_{i}, \mathbf{Z}$ is a diagonal matrix defined as $\mathbf{Z}=\operatorname{diag}_{(1 \leq i \leq m)}\left(\mathbf{Z}_{i}\right)$ where $\mathbf{Z}_{i}=\mathbf{1}_{n_{i}}$, and $\mathbf{v}=\left(v_{1}, \cdots, v_{m}\right)^{T}$. Matrices $\mathbf{P}$ and $\mathbf{D}$ represent the fixed and random effects of the $\mathrm{P}$-spline term for $p_{j \mid i}$, while $\mathbf{M}$ and $\mathbf{B}$ denote the fixed and random effects of the $\mathrm{P}$-spline term for $p_{i}$. Let $\sum_{i-1}^{m} n_{i}=n$, then we have

$$
\mathbf{P}=\left[\begin{array}{ccc}
p_{1 \mid 1} & \cdots & p_{1 \mid 1}^{h} \\
\vdots & & \vdots \\
p_{n_{m} \mid m} & \cdots & p_{n_{m} \mid m}{ }^{h}
\end{array}\right]_{n \times h}, \quad \mathbf{D}=\left[\begin{array}{ccc}
\left(p_{1 \mid 1}-q_{1}\right)_{+}^{h} & \cdots & \left(p_{1 \mid 1}-q_{k}\right)_{+}^{h} \\
\vdots & \vdots & \\
\left(p_{n_{m} \mid m}-q_{1}\right)_{+}^{h} & \cdots & \left(p_{n_{m} \mid m}-q_{k}\right)_{+}^{h}
\end{array}\right]_{n \times k},
$$




$$
\mathbf{M}=\left[\begin{array}{ccc}
p_{1} & \cdots & p_{1}^{l} \\
\vdots & \vdots & \vdots \\
p_{1} & \cdots & p_{1}^{l} \\
p_{2} & \cdots & p_{2}^{l} \\
\vdots & \vdots & \vdots \\
p_{2} & \cdots & p_{2}^{l} \\
\vdots & \vdots & \vdots \\
p_{m} & \cdots & p_{m}^{l} \\
\vdots & \vdots & \vdots \\
p_{m} & \cdots & p_{m}^{l}
\end{array}\right]_{n \times l} \quad \text { and } \quad \mathbf{B}=\left[\begin{array}{cccc}
\left(p_{1}-t_{1}\right)_{+}^{l} & \cdots & \left(p_{1}-t_{t}\right)_{+}^{l} \\
\left(p_{1}-t_{1}\right)_{+}^{l} & \cdots & \left(p_{1}-t_{t}\right)_{+}^{l} \\
\vdots & \vdots & \vdots \\
\left(p_{2}-t_{2}\right)_{+}^{l} & \cdots & \left(p_{2}-t_{t}\right)_{+}^{l} \\
\left(p_{2}-t_{2}\right)_{+}^{l} & \cdots & \left(p_{2}-t_{t}\right)_{+}^{l} \\
\vdots & \vdots & \vdots \\
\left(p_{m}-t_{1}\right)_{+}^{l} & \cdots & \left(p_{m}-t_{t}\right)_{+}^{l} \\
\vdots & \vdots & \vdots \\
\left(p_{m}-t_{1}\right)_{+}^{l} & \cdots & \left(p_{m}-t_{t}\right)_{+}^{l}
\end{array}\right]_{n \times t}
$$

Matrices $\mathbf{M}, \mathbf{P}$ and $\mathbf{X}$ are all fixed effects in the model and can be combined together in matrix $\mathbf{A}$ :

$$
\mathbf{A}=\left[\begin{array}{cccc|ccc|ccc}
1 & x_{11} & \cdots & x_{11}^{(p)} & p_{1 \mid 1} & \cdots & p_{1 \mid 1}{ }^{h} & p_{1} & \cdots & p_{1}{ }^{l} \\
\vdots & & \vdots & \vdots & \vdots & & \vdots & \vdots & & \vdots \\
1 & x_{m n_{m}} & \cdots & x_{m n_{n}}^{(p)} & p_{n_{m} \mid m} & \cdots & p_{n_{m} \mid m}{ }^{h} & p_{m} & \cdots & p_{m}{ }^{l}
\end{array}\right]_{n \times(p+h+l+1)}
$$

Let $\boldsymbol{\beta}^{\star}=\left(\beta_{0}, \beta_{1}, \ldots \beta_{p}, \alpha_{1}, \ldots, \alpha_{h}, \lambda_{1}, \ldots, \lambda_{l}\right)^{T}$, then the linear mixed model can be expressed as

$$
\mathbf{Y}=\mathbf{A} \boldsymbol{\beta}^{\star}+\mathbf{D} \boldsymbol{\gamma}+\mathbf{B} \boldsymbol{\tau}+\mathbf{Z} \mathbf{v}+\mathbf{e}
$$

Assuming $\boldsymbol{\gamma} \sim\left(\mathbf{0}, \sigma_{k}^{2} \mathbf{I}_{k}\right), \boldsymbol{\tau} \sim\left(\mathbf{0}, \sigma_{t}^{2} \mathbf{I}_{t}\right), \mathbf{v} \sim\left(\mathbf{0}, \sigma_{v}^{2} \mathbf{I}_{m}\right)$ and $\mathbf{e} \sim\left(\mathbf{0}, \sigma_{e}^{2} \mathbf{I}_{n}\right)$ we can find the best linear unbiased estimator (BLUE) of $\boldsymbol{\beta}^{\star}$ and the BLUP of $\boldsymbol{\gamma}, \boldsymbol{\tau}, \mathbf{v}$ by solving "mixed model" equations as shown in Henderson (1963): 


$$
\left[\begin{array}{cccc}
\frac{1}{\sigma_{e}^{2}} \mathbf{A}^{T} \mathbf{A} & \frac{1}{\sigma_{e}^{2}} \mathbf{A}^{T} \mathbf{D} & \frac{1}{\sigma_{e}^{2}} \mathbf{A}^{T} \mathbf{B} & \frac{1}{\sigma_{e}^{2}} \mathbf{A}^{T} \mathbf{Z} \\
\frac{1}{\sigma_{e}^{2}} \mathbf{D}^{T} \mathbf{A} & \frac{1}{\sigma_{\gamma}^{2}} \mathbf{I}_{k}+\frac{1}{\sigma_{e}^{2}} \mathbf{D}^{T} \mathbf{D} & \frac{1}{\sigma_{e}^{2}} \mathbf{D}^{T} \mathbf{B} & \frac{1}{\sigma_{e}^{2}} \mathbf{D}^{T} \mathbf{Z} \\
\frac{1}{\sigma_{e}^{2}} \mathbf{B}^{T} \mathbf{A} & \frac{1}{\sigma_{e}^{2}} \mathbf{B}^{T} \mathbf{D} & \frac{1}{\sigma_{t}^{2}} \mathbf{I}_{t}+\frac{1}{\sigma_{e}^{2}} \mathbf{B}^{T} \mathbf{B} & \frac{1}{\sigma_{e}^{2}} \mathbf{B}^{T} \mathbf{Z} \\
\frac{1}{\sigma_{e}^{2}} \mathbf{Z}^{T} \mathbf{A} & \frac{1}{\sigma_{e}^{2}} \mathbf{Z}^{T} \mathbf{D} & \frac{1}{\sigma_{e}^{2}} \mathbf{Z}^{T} \mathbf{B} & \frac{1}{\sigma_{v}^{2}} \mathbf{I}_{m}+\frac{1}{\sigma_{e}^{2}} \mathbf{Z}^{T} \mathbf{Z}
\end{array}\right]\left[\begin{array}{c}
\boldsymbol{\beta}^{\star} \\
\boldsymbol{\gamma} \\
\boldsymbol{\tau} \\
\mathbf{v}
\end{array}\right]=\left[\begin{array}{c}
\frac{1}{\sigma_{e}^{2}} \mathbf{A}^{T} \mathbf{y} \\
\frac{1}{\sigma_{e}^{2}} \mathbf{D}^{T} \mathbf{y} \\
\frac{1}{\sigma_{e}^{2}} \mathbf{B}^{T} \mathbf{y} \\
\frac{1}{\sigma_{e}^{2}} \mathbf{Z}^{T} \mathbf{y}
\end{array}\right]
$$

Let $\mathbf{W}$ be the inverse of the first matrix on the left side of (3.2), then the solution of (3.2) can be expressed as

$$
\left[\begin{array}{c}
\tilde{\boldsymbol{\beta}}^{\star} \\
\tilde{\boldsymbol{\gamma}} \\
\tilde{\boldsymbol{\tau}} \\
\tilde{\mathbf{v}}
\end{array}\right]=\left[\begin{array}{c}
\frac{1}{\sigma_{e}^{2}}\left(W_{11} \mathbf{A}^{T}+W_{12} \mathbf{D}^{T}+W_{13} \mathbf{B}^{T}+W_{14} \mathbf{Z}^{T}\right) \mathbf{y} \\
\frac{1}{\sigma_{e}^{2}}\left(W_{21} \mathbf{A}^{T}+W_{22} \mathbf{D}^{T}+W_{23} \mathbf{B}^{T}+W_{24} \mathbf{Z}^{T}\right) \mathbf{y} \\
\frac{1}{\sigma_{e}^{2}}\left(W_{31} \mathbf{A}^{T}+W_{32} \mathbf{D}^{T}+W_{33} \mathbf{B}^{T}+W_{34} \mathbf{Z}^{T}\right) \mathbf{y} \\
\frac{1}{\sigma_{e}^{2}}\left(W_{41} \mathbf{A}^{T}+W_{42} \mathbf{D}^{T}+W_{43} \mathbf{B}^{T}+W_{44} \mathbf{Z}^{T}\right) \mathbf{y}
\end{array}\right]
$$

where $W_{i j}$ 's with $i=1, \cdots, 4, j=1, \cdots, 4$ are elements of matrix $\mathbf{W}$.

Repersentation (3.3) depends on unknown variance components, $\boldsymbol{\sigma}=$ $\left(\sigma_{e}^{2}, \sigma_{\gamma}^{2}, \sigma_{t}^{2}, \sigma_{v}^{2}\right)^{T}$, which can be estimated iteratively by fixed-point equations

$$
\begin{aligned}
\sigma_{t}^{2} & =\sum_{t=1}^{T} \frac{\tilde{\tau}_{t}^{2}}{t-\left(\operatorname{tr}\left(W_{33}\right) / \sigma_{\tau}^{2}\right)} \\
\sigma_{\gamma}^{2} & =\sum_{k=1}^{K} \frac{\tilde{\gamma}_{k}^{2}}{k-\left(\operatorname{tr}\left(W_{22}\right) / \sigma_{\gamma}^{2}\right)} \\
\sigma_{v}^{2} & =\sum_{i=1}^{m} \frac{\tilde{v}_{i}^{2}}{m-\left(\operatorname{tr}\left(W_{44}\right) / \sigma_{v}^{2}\right)}
\end{aligned}
$$




$$
\sigma_{e}^{2}=\sum_{i=1}^{m} \sum_{j=1}^{n_{i}} \frac{\tilde{e}_{i j}^{2}}{n-\left(p+\left(t-\operatorname{tr}\left(W_{33}\right) / \sigma_{t}^{2}\right)\right)+\left(m-\left(\operatorname{tr}\left(W_{44}\right) / \sigma_{v}^{2}\right)+\left(k-\left(\operatorname{tr}\left(W_{22}\right) / \sigma_{\gamma}^{2}\right)\right)\right.}
$$

where $\tilde{v}_{i}, \tilde{\gamma}_{k}, \tilde{\tau}_{t}$ are obtained from (3.3) and $\tilde{e}_{i j}$ is equal to

$$
\tilde{e}_{i j}=y_{i j}-\mathbf{x}_{i j}^{T} \tilde{\boldsymbol{\beta}}^{\star}-\mathbf{D}_{i j}^{T} \tilde{\boldsymbol{\gamma}}-\mathbf{B}_{i}^{T} \tilde{\boldsymbol{\tau}}-\tilde{v}_{i}
$$

Substituting (3.4), (3.5), (3.6) and (3.7) into (3.3), the EBLUP estimators of small area means for sampled areas $\left(I_{i}=1\right)$ can be obtained by

$$
\hat{\mu}_{i}=\frac{1}{N_{i}}\left(\sum_{j \in s_{i}} y_{i j}+\sum_{j \in \bar{s}_{i}} \hat{y}_{i j}\right) \quad i=1, \cdots, m
$$

where $s_{i}$ and $\bar{s}_{i}$ represent the set of sampled units and the set of non-sampled units in the selected area $i$, and $\hat{y}_{i j}$ denotes the predictor of $y_{i j}$ for $j \in \bar{s}_{i}$ which is in the form of

$$
\hat{y}_{i j}=\mathbf{A}_{i j}^{T} \hat{\boldsymbol{\beta}}^{\star}+\mathbf{B}_{i}^{T} \hat{\boldsymbol{\tau}}+\mathbf{D}_{i j}^{T} \hat{\gamma}+\hat{v}_{i}
$$

where $\hat{\boldsymbol{\beta}}^{\star}$ denotes the empirical BLUE of $\boldsymbol{\beta}^{\star}, \hat{\boldsymbol{\gamma}}, \hat{\boldsymbol{\tau}}$ and $\hat{v}_{i}$ represent EBLUP of $\boldsymbol{\gamma}, \boldsymbol{\tau}$ and $v_{i}$ respectively, $\mathbf{A}_{i j}=\left(1, x_{i j}, \cdots, x_{i j}^{p}, p_{j \mid i}, \cdots, p_{j \mid i}^{h}, p_{i}, \cdots, p_{i}^{l}\right)^{T}, \mathbf{B}_{i j}=$ $\left(\left(p_{i}-t_{1}\right)_{+}^{l}, \cdots,\left(p_{i}-t_{t}\right)_{+}^{l}\right)^{T}$ and $\mathbf{D}_{i j}=\left(\left(p_{j \mid i}-q_{1}\right)_{+}^{h}, \cdots,\left(p_{j \mid i}-q_{k}\right)_{+}^{h}\right)^{T}$.

For the non-sampled areas $\left(I_{i}=0\right)$, the EBLUP estimators of the area means consist of the predictors of $y_{i j}$ only.

$$
\hat{\mu}_{i}=\frac{1}{N_{i}}\left(\sum_{j \in U_{i}} \hat{y}_{i j}\right), \quad i=m+1, \cdots, M
$$


where $U_{i}$ denotes the set of all units in a non-selected area and $\hat{y}_{i j}$ is the predictor of those units. The value of $\hat{y}_{i j}$ depends on the availability of the $p_{i}$ and $p_{j \mid i}$. There will be three possible cases:

1. Assuming both $p_{i}$ and $p_{j \mid i}$ are known for the non-sampled areas, the $\hat{y}_{i j}$ include spline terms based on both $p_{j \mid i}$ and $p_{i}$. Suppose that the model holds for non-sampled areas $i=m+1, \cdots, M$, then we use a synthetic predictor defined as

$$
\hat{y}_{i j}=\mathbf{A}_{i j}^{T} \hat{\boldsymbol{\beta}}^{\star}+\mathbf{B}_{i}^{T} \hat{\boldsymbol{\tau}}+\mathbf{D}_{i j}^{T} \hat{\boldsymbol{\gamma}}, \quad i=m+1, \cdots, M, \quad j=1, \cdots, N_{i},
$$

where $\hat{\boldsymbol{\beta}}^{\star}$ denotes the empirical BLUE of $\boldsymbol{\beta}^{\star}=\left(\beta_{0}, \beta_{1}, \ldots \beta_{p}, \alpha_{1}, \ldots, \alpha_{h}, \lambda_{1}, \ldots, \lambda_{l}\right)^{T}$, $\hat{\boldsymbol{\gamma}}, \hat{\boldsymbol{\tau}}$ represent the EBLUP of $\boldsymbol{\gamma}$, and $\boldsymbol{\tau}, \mathbf{A}_{i j}=\left(1, x_{i j}, \cdots, x_{i j}^{p}, p_{j \mid i}, \cdots, p_{j \mid i}^{h}, p_{i}, \cdots, p_{i}^{l}\right)^{T}$, $\mathbf{B}_{i j}=\left(\left(p_{i}-t_{1}\right)_{+}^{l}, \cdots,\left(p_{i}-t_{t}\right)_{+}^{l}\right)^{T}$ and $\mathbf{D}_{i j}=\left(\left(p_{j \mid i}-q_{1}\right)_{+}^{h}, \cdots,\left(p_{j \mid i}-q_{k}\right)_{+}^{h}\right)^{T}$.

2. Assuming $p_{i}$ are known for non-sampled, but $p_{j \mid i}$ are not known, then $\hat{y}_{i j}$ only includes the P-spline term of $p_{i}$.

$$
\hat{y}_{i j}=\mathbf{A}_{i j}^{T} \hat{\boldsymbol{\beta}}^{\star}+\mathbf{B}_{i}^{T} \hat{\boldsymbol{\tau}}, \quad i=m+1, \cdots, M, \quad j=1, \cdots, N_{i},
$$

where $\hat{\boldsymbol{\beta}}^{\star}$ denotes the empirical BLUE of $\boldsymbol{\beta}^{\star}=\left(\beta_{0}, \beta_{1}, \cdots, \beta_{p}, \lambda_{1}, \cdots, \lambda_{l}\right)^{T}$, $\hat{\boldsymbol{\tau}}$ is the EBLUP of $\boldsymbol{\tau}, \mathbf{A}_{i j}=\left(1, x_{i j}, \cdots, x_{i j}^{p}, p_{i}, \cdots, p_{i}^{l}\right)^{T}$ and $\mathbf{B}_{i}=\left(\left(p_{i}-\right.\right.$ $\left.\left.t_{1}\right)_{+}^{l}, \cdots,\left(p_{i}-t_{t}\right)_{+}^{l}\right)^{T}$.

3. Assuming both $p_{i}$ and $p_{j \mid i}$ are not known for the non-sampled areas, the predictor of $y_{i j}$ is only based on known auxiliary information $\mathbf{x}_{i j}$ in non- 
sampled areas.

$$
\hat{y}_{i j}=\mathbf{A}_{i j}^{T} \hat{\boldsymbol{\beta}}^{\star}, \quad i=m+1, \cdots, M, \quad j=1, \cdots, N_{i},
$$

where $\hat{\boldsymbol{\beta}}^{\star}=\left(\hat{\beta}_{0}, \hat{\beta}_{1}, \cdots, \hat{\beta}_{p}\right)^{T}, \mathbf{A}_{i j}=\left(1, x_{i j}, \cdots, x_{i j}^{p}\right)^{T}$.

\subsubsection{Case II: stage 1 informative, stage 2 not informative}

For Case II, areas (i.e., PSU's) are selected using probability proportional to size (PPS) sampling while units in selected areas are drawn using simple random sampling (SRS). Hence, with respect to the proposed method, we have one Pspline term for $p_{i}, \mathcal{G}\left(p_{i}\right)$. The sample model has the following form:

$$
y_{i j}=\mathbf{x}_{i j}^{T} \boldsymbol{\beta}+\mathcal{G}\left(p_{i}\right)+e_{i j}, \quad i=1, \cdots, m, \quad j=1, \cdots, n_{i},
$$

where $\mathcal{G}\left(p_{i}\right)=\lambda_{1} p_{i}+\lambda_{2} p_{i}^{2}+\cdots+\lambda_{l} p_{i}^{l}+\sum_{t=1}^{T} \tau_{t}\left(p_{i}-t_{t}\right)_{+}^{l}$.

Similar to Case I, for sampled areas $\left(I_{i}=1\right)$, the EBLUP of small area means is:

$$
\hat{\mu}_{i}=\frac{1}{N_{i}}\left(\sum_{j \in s_{i}} y_{i j}+\sum_{j \in \bar{s}_{i}} \hat{y}_{i j}\right), \quad i=1, \cdots, m
$$

where $s_{i}$ and $\bar{s}_{i}$ represent the set of sampled units and the set of non-sampled units in the selected area $i$, and $\hat{y}_{i j}$ denotes the predictor of $y_{i j}$ for $j \in \bar{s}_{i}$ which is in the form of

$$
\hat{y}_{i j}=\mathbf{A}_{i j}^{T} \hat{\boldsymbol{\beta}}^{\star}+\mathbf{B}_{i}^{T} \hat{\boldsymbol{\tau}}+\hat{v}_{i}
$$

where $\hat{\boldsymbol{\beta}}^{\star}, \hat{\boldsymbol{\tau}}$, and $\hat{v}_{i}$ are calculated by solving an adjusted form of (3.3), where $\mathbf{W}$ is a $3 \times 3$ matrix after dropping elements associated with $p_{j \mid i}$. 


$$
\begin{aligned}
& \boldsymbol{\beta}^{\star}=\left(\beta_{0}, \beta_{1}, \cdots, \beta_{p}, \lambda_{1}, \cdots, \lambda_{l}\right), \mathbf{A}_{i j}=\left(1, x_{i j}, \cdots, x_{i j}^{p}, p_{i}, \cdots, p_{i}^{l}\right)^{T} \text { and } \mathbf{B}_{i}= \\
& \left(\left(p_{i}-t_{1}\right)_{+}^{l}, \cdots,\left(p_{i}-t_{t}\right)_{+}^{l}\right)^{T} .
\end{aligned}
$$

For the non-sampled areas $\left(I_{i}=0\right)$, assuming that the model holds for nonsampled areas, the EBLUP consists of the predictors of $y_{i j}$ only, which depends on the availability of $p_{i}$ in non-sampled areas. We have

$$
\hat{\mu}_{i}=\frac{1}{N_{i}}\left(\sum_{j \in U_{i}} \hat{y}_{i j}\right) \quad i=m+1, \cdots, M
$$

where $U_{i}$ denotes the set all units in non-selected area $i$ and $\hat{y}_{i j}$ is the predictor of those units. The value of $\hat{y}_{i j}$ depends on the availability of the $p_{i}$.

1. Assuming that $p_{i}$ is known for the non-sampled areas, $y_{i j}$ is predicted as

$$
\hat{y}_{i j}=\mathbf{A}_{i j}^{T} \hat{\boldsymbol{\beta}}^{\star}+\mathbf{B}_{i}^{T} \hat{\boldsymbol{\tau}}, \quad i=m+1, \cdots, M, \quad j=1, \cdots, N_{i},
$$

where $\hat{\boldsymbol{\beta}}^{\star}=\left(\hat{\beta}_{0}, \hat{\beta}_{1}, \cdots, \hat{\beta}_{p}, \hat{\lambda}_{1}, \cdots, \hat{\lambda}_{l}\right)^{T}, \mathbf{A}_{i j}=\left(1, x_{i j}, \cdots, x_{i j}^{p}, p_{i}, \cdots, p_{i}^{l}\right)^{T}$ and $\mathbf{B}_{i}=\left(\left(p_{i}-t_{1}\right)_{+}^{l}, \cdots,\left(p_{i}-t_{t}\right)_{+}^{l}\right)^{T}$.

2. Assuming that $p_{i}$ of the non-sampled areas are unknown, $\hat{y}_{i j}$ only relies on known covariates values $\mathbf{x}_{i j}$ in non-sampled areas.

$$
\hat{y}_{i j}=\mathbf{A}_{i j}^{T} \hat{\boldsymbol{\beta}}^{\star}, \quad i=m+1, \cdots, M, \quad j=1, \cdots, N_{i},
$$

where $\hat{\boldsymbol{\beta}}^{\star}=\left(\hat{\beta}_{0}, \hat{\beta}_{1}, \cdots, \hat{\beta}_{p}\right)^{T}$ and $\mathbf{A}_{i j}=\left(1, x_{i j}, \cdots, x_{i j}^{p}\right)^{T}$. 


\subsubsection{Case III: stage 1 not informative, stage 2 informative}

For Case III, areas are selected using SRS, however units in the selected areas are drawn using PPS sampling. So, we will have one P-spline term that adjusts for the informativeness of the second stage of sampling. The sample model has the following form:

$$
y_{i j}=\mathbf{x}_{i j}^{T} \boldsymbol{\beta}+v_{i}+\mathcal{G}\left(p_{j \mid i}\right)+e_{i j}, \quad i=1, \cdots, m, \quad j=1, \cdots, n_{i},
$$

where $\mathcal{G}\left(p_{j \mid i}\right)=\alpha_{1} p_{j \mid i}+\alpha_{1} p_{j \mid i}^{2}+\cdots+\alpha_{h} p_{j \mid i}^{h}+\sum_{k=1}^{K} \gamma_{k}\left(p_{j \mid i}-q_{k}\right)_{+}^{h}$.

For sampled areas $\left(I_{i}=1\right)$, the EBLUP of small area means is:

$$
\hat{\mu}_{i}=\frac{1}{N_{i}}\left(\sum_{j \in s_{i}} y_{i j}+\sum_{j \in \bar{s}_{i}} \hat{y}_{i j}\right), \quad i=1, \cdots, m
$$

where

$$
\hat{y}_{i j}=\mathbf{A}_{i j}^{T} \hat{\boldsymbol{\beta}}^{\star}+\mathbf{D}_{i j}^{T} \hat{\gamma}+\hat{v}_{i}
$$

with $\left.\mathbf{A}_{i j}=\left(1, x_{i j}, \cdots, x_{i j}^{(p)}, p_{j \mid i}, \cdots, p_{j \mid i}^{h}\right)^{T}, \mathbf{D}_{i j}=\left(\left(p_{j \mid i}-q_{1}\right)_{+}^{h}, \cdots, p_{j \mid i}-q_{k}\right)_{+}^{h}\right)^{T}$, $\hat{\boldsymbol{\beta}}^{\star}$ denotes the empirical BLUE of $\boldsymbol{\beta}^{\star}, \hat{\boldsymbol{\gamma}}$, and $\hat{v}_{i}$ represent EBLUP of $\boldsymbol{\gamma}$, and $v_{i}$. $\hat{\boldsymbol{\beta}}^{\star}, \hat{\boldsymbol{\gamma}}$, and $\hat{v}_{i}$ are calculated by solving an adopted form of (3.3). More precisely, here elements associated with $p_{i}$ are dropped and $\mathbf{W}$ is a $3 \times 3$ matrix.

For the non-sampled areas $\left(I_{i}=0\right)$, assuming that model holds for nonsampled areas, the EBLUP of small area means is based on predictors of $y_{i j}$ only, which depends on the availability of $p_{j \mid i}$ in non-sampled areas.

$$
\hat{\mu}_{i}=\frac{1}{N_{i}}\left(\sum_{j \in U_{i}} \hat{y}_{i j}\right), \quad i=m+1, \cdots, M, \quad j=1, \cdots, N_{i}
$$


1. Assuming that $p_{j \mid i}$ is known for non-sampled areas,

$$
\hat{y}_{i j}=\mathbf{A}_{i j}^{T} \hat{\boldsymbol{\beta}}^{\star}+\mathbf{D}_{i j}^{T} \hat{\gamma}, \quad i=m+1, \cdots, M, \quad j=1, \cdots, N_{i}
$$

where $\mathbf{A}_{i j}=\left(1, x_{i j}, \cdots, x_{i j}^{p}, p_{j \mid i}, \cdots, p_{j \mid i}^{h}\right)^{T}, \mathbf{D}_{i j}=\left(\left(p_{j \mid i}-q_{1}\right)_{+}^{h}, \cdots,\left(p_{j \mid i}-\right.\right.$ $\left.\left.q_{k}\right)_{+}^{h}\right)^{T}$ and $\boldsymbol{\beta}^{\star}=\left(\beta_{0}, \beta_{1}, \ldots \beta_{p}, \lambda_{1}, \ldots, \lambda_{l}\right)^{T}$.

2. Assuming that $p_{j \mid i} \mid I_{i}=0$ is unknown, the estimator for non-sampled areas is

$$
\hat{y}_{i j}=\mathbf{A}_{i j}^{T} \hat{\boldsymbol{\beta}}^{\star}, \quad i=m+1, \cdots, M, \quad j=1, \cdots, N_{i},
$$

where $\hat{\boldsymbol{\beta}}^{\star}=\left(\hat{\beta}_{0}, \hat{\beta}_{1}, \cdots, \hat{\beta}_{p}\right), \mathbf{A}_{i j}=\left(1, x_{i j}, \cdots, x_{i j}^{p}\right)^{T}$.

To some extent, the derivation of EBLUP for Case III is similar to one-stage sampling discussed in Chapter 2.

\subsection{Mean squared error}

Similar to Chapter 2, we use a conditional bootstrap method for estimating the MSE, following Rao et al. (2014). Assuming that both stages of sampling are informative, the inference is conditioned on $\hat{\gamma}$ and $\hat{\boldsymbol{\tau}}$.

We first estimate $\hat{\boldsymbol{\beta}}^{\star}, \hat{\boldsymbol{\gamma}}, \hat{\boldsymbol{\tau}}$, as well as the variance components using the

original sample. Then, we generate $v_{i}^{\bullet} \sim N\left(0, \hat{\sigma}_{v}^{2}\right)$ and $e_{i j}^{\bullet} \sim N\left(0, \hat{\sigma}_{e}^{2}\right)$, and obtain the bootstrap responses as follows, assuming that both $p_{i}$ and $p_{j \mid i}$ are known for the non-sampled areas

$$
y_{i j}^{\bullet}=\mathbf{A}_{i j}^{T} \hat{\boldsymbol{\beta}}^{\star}+\mathbf{B}_{i}^{T} \hat{\boldsymbol{\tau}}+\mathbf{D}_{i j}^{T} \hat{\boldsymbol{\gamma}}+v_{i}^{\bullet}+e_{i j}^{\bullet} \quad i=1, \cdots, M, \quad j=1, \cdots, N_{i}
$$


Using the corresponding sample data $\left\{\left(y_{i j}^{\bullet}, \mathbf{A}_{i j}, \mathbf{B}_{i}, \mathbf{D}_{i j}\right), j \in s_{i} \quad i=1, \cdots m\right\}$, we obtain the bootstrap estimates $\hat{\boldsymbol{\beta}}^{\bullet}, \hat{\boldsymbol{\tau}}^{\bullet}, \hat{\boldsymbol{\gamma}}^{\bullet}, \hat{v}_{i}^{\bullet}$. The predicted values $\hat{y}_{i j}^{\bullet}$ for $j \in \bar{s}_{i}$ are given by:

$$
\hat{y}_{i j}^{\bullet}=\mathbf{A}_{i j}^{T} \hat{\boldsymbol{\beta}}^{\bullet}+\mathbf{B}_{i}^{T} \hat{\boldsymbol{\tau}}^{\bullet}+\mathbf{D}_{i j}^{T} \hat{\boldsymbol{\gamma}}^{\bullet}+\hat{v}_{i}^{\bullet}
$$

The bootstrap EBLUP of the $\bar{Y}_{i}$ for sampled areas can be found by

$$
\hat{\mu}_{i}^{\bullet}=\frac{1}{N_{i}}\left(\sum_{j \in s_{i}} y_{i j}^{\bullet}+\sum_{j \in \bar{s}_{i}} \hat{y}_{i j}^{\bullet}\right), \quad i=1, \cdots, m
$$

where $\hat{y}_{i j}^{\bullet}$ is derived from (3.12).

For non-sampled areas, the bootstrap EBLUP consists of the predicted values $\hat{y}_{i j}^{\bullet}$ only.

$$
\hat{\mu}_{i}^{\bullet}=\frac{1}{N_{i}}\left(\sum_{j \in U_{i}} \hat{y}_{i j}^{\bullet}\right), \quad i=m+1, \cdots, M,
$$

where $\hat{y}_{i j}^{\bullet}, \quad i=m+1, \cdots, M$ can be found by (3.9).

We repeat the above procedure $\mathrm{B}$ times, to find the conditional bootstrap estimator of MSE of the EBLUP of $\bar{Y}_{i}$ as follows:

$$
\hat{M}_{\text {boot }}\left(\hat{\mu}_{i}\right)=\frac{1}{B} \sum_{b=1}^{B}\left(\hat{\mu}_{i}^{\bullet}(b)-\bar{Y}_{i}^{\bullet}(b)\right)^{2}, \quad i=1, \cdots, M .
$$

where $\hat{\mu}_{i}^{\bullet}(b)$ and $\bar{Y}_{i}^{\bullet}(b)$ are values of $\hat{\mu}_{i}$ and $\bar{Y}_{i}$ for the $b^{\text {th }}$ bootstrap replicate.

The procedure described above is for Case I, where both stages of sampling are informative. For Case II or Case III, we need to adjust (3.11) and (3.12) accordingly and use appropriate equations derived in Section 3.2.2 and Section 3.2.3. 
The drawback of the proposed for estimation of MSE is that $p_{i}$ and $p_{j \mid i}$ values should be known for all units in the population, which may not be feasible in practice.

\subsection{Simulation study}

To illustrate the performance of the proposed method under two-stage sampling, we designed a small design-based simulation study under two scenarios: 1) Case I, where both stages of sampling are informative, and 2) Case II, where the first stage (i.e., selection of areas) is informative but the second stage is non-informative and units in selected areas are selected by SRS.

The population is generated as follows:

1. Generate area sizes, $N_{i}=100$ for $i=1, \ldots, 150$ areas and covariate $x_{i j}$ from gamma with mean 10 and variance 50

2. Generate population random area effects, $v_{i}$ from $N(0,0.5)$

3. Generate $y_{i j}$ values using $y_{i j}=\beta_{0}+\beta_{1} x_{i j}+v_{i}+e_{i j}$ where $e_{i j} \sim N(0,2)$, $\beta_{0}=1$ and $\beta_{1}=1$. To avoid extreme selection probabilities, random effects $v_{i}$ and $e_{i j}$ are truncated at $\pm 2.5 \sigma_{v}$ and $\pm 2.5 \sigma_{e}$ respectively.

It should be noted that $x_{i j}$ values are held fixed throughout the simulation.

\subsubsection{Case I: both stages are informative}

We select 90 areas using systematic PPS sampling with probabilities proportional

to size measures $b_{i}=\operatorname{Int}\left[50 \times \exp \left(-\left(v_{i} / \sigma_{v}\right)\right)\right]$, where $\operatorname{Int}[x]$ gives the integer part 
of $x$ defined as follows

$$
\text { Int }[x]=\lfloor x\rfloor \quad \text { if } \quad x>0 .
$$

For systematic PPS sampling, we used function "pps.sampling" in R-package "samplingbook" (Manitz, 2012). The choice of $b_{i}$ makes the area selection informative. Then, we select $n_{i}, i=1, \ldots 90$ units in each selected area using the RaoSampford method (Rao 1965 and Sampford 1967) with probabilities proportional to size measures $\left.b_{i j}=\exp \left[-\left(v_{i}+e_{i j}\right) / 2+\delta_{i j} / 5\right) / 3\right]$ where $\delta_{i j} \sim N(0,1)$. The sample size in sampled areas are $n_{1}=n_{2}=\cdots=n_{30}=5, n_{31}=n_{32}=\cdots=n_{60}=7$ and $n_{61}=n_{62}=\cdots=n_{90}=9$. We repeat the sampling step for $R=2000$ times. For each simulated sample, we compute small area means, following the steps mentioned in Section 3.2.1.

To evaluate the impact of the spline degree on the performance of the method, we test the P-spline method under two setups:

1. Linear P-spline with $h=1$

2. Quadratic P-spline with $h=2$

More precisely, for the linear case the $\mathcal{G}_{2}\left(p_{j \mid i}\right)$ in (3.1) is defined as follows:

$$
m_{2}\left(p_{j \mid i}\right)=\alpha_{1} p_{j \mid i}+\sum_{k=1}^{30} \gamma_{k}\left(p_{j \mid i}-q_{k}\right)_{+}
$$

For the quadratic case we will have :

$$
m_{2}\left(p_{j \mid i}\right)=\alpha_{1} p_{j \mid i}+\alpha_{2} p_{j \mid i}^{2}+\sum_{k=1}^{30} \gamma_{k}\left(p_{j \mid i}-q_{k}\right)_{+}^{2}
$$


We set the number of knots on $p_{j \mid i}$ and the number of knots on $p_{i}$ equal to $\mathrm{K}=30$ and $\mathrm{T}=18$, respectively. For both $\mathcal{G}_{1}\left(p_{i}\right)$ and $\mathcal{G}_{2}\left(p_{j \mid i}\right)$, the knots are placed at the quantile of the $p_{i}$ 's and $p_{j \mid i}$ 's values. Regarding the choice of number of knots, $K$ and $T$, and the location of the knots, we followed Ruppert et al. (2003).

In Table 3.1 below, the performance of the P-spline method under linear and quadratic splines is compared to the method proposed by Pfeffermann and Sverchkov (2007), as well as the ordinary EBLUP. The method by Pfeffermann and Sverchkov (denoted by "PS" here), is described in Section 2.2.1 for the special case of $m=M$. For non-sampled areas, PS estimator is

$$
\hat{\bar{Y}}_{i}=\overline{\mathbf{X}}_{i}^{T} \hat{\boldsymbol{\beta}}+\hat{b} \hat{\sigma}_{e}^{2}+\left[\sum_{i \in s}\left(w_{i}-1\right) \hat{v}_{i} / \sum_{i \in s}\left(w_{i}-1\right)\right]
$$

where $w_{i}=\pi_{i}^{-1}, \hat{b}$ is obtained by regressing the sampling weights $w_{j \mid i}$ on $k_{i} \exp \left(\mathbf{x}_{i j}^{T} \mathbf{a}+b y_{i j}\right), \hat{v}_{i}-\hat{\gamma}_{i}\left(y_{i}-\bar{X}_{i}^{T} \hat{\boldsymbol{\beta}}\right)$ with $\hat{\gamma}_{i}=\hat{\sigma}_{v}^{2} /\left(\hat{\sigma}_{v}^{2}+n_{i}^{-1} \hat{\sigma}_{e}^{2}\right)$.

For each method, we computed average absolute bias $(\overline{A B})$ and average RMSE $(\overline{R M S E})$. For sampled areas $\left(I_{i}=1\right)$ :

$$
\begin{gathered}
\overline{A B}=\frac{1}{m} \sum_{i=1}^{m} \frac{\left|\sum_{r=1}^{R} D_{i}^{(r)}\left(\hat{\bar{Y}}_{i}^{(r)}-\bar{Y}_{i}^{(r)}\right)\right|}{\sum_{r=1}^{R} D_{i}^{(r)}}, \\
\overline{R M S E}=\frac{1}{m} \sum_{i=1}^{m} \sqrt{\frac{\sum_{r=1}^{R} D_{i}^{(r)}\left(\hat{\bar{Y}}_{i}^{(r)}-\bar{Y}_{i}^{(r)}\right)^{2}}{\sum_{r=1}^{R} D_{i}^{(r)}}},
\end{gathered}
$$

where $D_{i}^{(r)}=1$ if area $i$ is sampled in simulation $\mathrm{r}$ and $D_{i}^{(r)}=0$ otherwise, $\hat{\bar{Y}}_{i}^{(r)}$ is the predictor of area $i$ of in $r$ simulation obtained from (3.8), and $\bar{Y}_{i}^{(r)}$ is the true mean of area $i$ for the $r^{\text {th }}$ simulated sample. 
Similarly, for non-sampled areas $\left(I_{i}=0\right)$ we average over non-sampled areas $(M-m)$ and replace $D_{i}^{(r)}$ with $\left(1-D_{i}^{(r)}\right)$ in (3.15) and (3.16).

Table 3.1: Average absolute bias and average RMSE for Case I

\begin{tabular}{||lccccc||}
\hline$I_{i}$ & Performance measure & Ordinary & PS & P-spline (linear) & P-spline (quadratic) \\
\hline \hline 1 & $\overline{A B}$ & 0.455 & 0.038 & 0.253 & 0.114 \\
& $\overline{R M S E}$ & 0.558 & 0.326 & 0.273 & 0.135 \\
\hline 0 & $\overline{A B}$ & 1.361 & 0.313 & 0.508 & 0.134 \\
& $\overline{R M S E}$ & 1.445 & 0.599 & 0.639 & 0.411 \\
\hline
\end{tabular}

Similar to the case of one-stage sampling (shown in the previous chapter), ignoring the informative sampling scheme results in large prediction bias. In particular, the $\overline{A B}$ of the ordinary EBLUP is relatively large compared to the P-spline method. For the sampled areas, the method proposed by Pfeffermann and Sverchkov performs better in terms of bias. The $\overline{A B}$ of the quadratic Pspline method for sampled areas is almost three times of the $\overline{A B}$ in Pfeffermann and Sverchkov's method. However, for non-sampled areas the P-spline method provides better results in terms of $\overline{A B}$ and $\overline{R M S E}$. The simulation results suggest that using quadratic form reduces the $\overline{A B}$ and average $\overline{R M S E}$ of the P-spline method, compared to the linear P-spline.

It should be noted that in this simulation, we have assumed that $p_{i}$ 's and $p_{j \mid i}$ 's are known for non-sampled areas. In practice, this assumption holds if we have access to a comprehensive sampling frame that contains some basic information needed for generating size measures for all units. In this situation, we can construct synthetic $p_{j \mid i}$ 's for units in non-sampled areas. In the absence of an 
inclusive sampling frame, we do not have much information about the $p_{j \mid i}$ 's of the non-sampled areas.

\subsubsection{Case II: stage 1 informative, stage 2 not informative}

For the second simulation, we generated the population in the same way as in the first simulation. This time, we select 90 areas using systematic PPS sampling with probabilities proportional to size measures $b_{i}=\operatorname{Int}\left[50 \times \exp \left(-\left(v_{i} / \sigma_{v}\right)\right)\right]$. Then, we draw a sample of $n_{i}$ units from each selected area using SRS where $n_{1}=n_{2}=$ $\cdots=n_{30}=5, n_{31}=n_{32}=\cdots=n_{60}=7$ and $n_{61}=n_{62}=\cdots=n_{90}=9$.

For the P-spline method, we set $l=1$ (linear model) and $T=12$ knots. The knots are placed at the quantile of the $p_{i}$ 's values. Assuming that $p_{i}$ is known for all areas $i=1, \ldots, 150$, we compute the small area means using equations in Section 3.2.2. We compared the performance of the P-spline method with the ordinary EBLUP and the augmented method proposed by Verret et al. (2015), discussed in section (2.2.2). Verret et al.'s method is defined under one-stage sampling. The following is a natural extension of their method to a special case of PPS-SRS two-stage sampling.

The augmented sample model due to Verret et al. (2015) will have the following form for PPS-SRS sampling:

$$
y_{i j}=\mathbf{x}_{i j}^{T} \boldsymbol{\beta}+\delta g\left(p_{i}\right)+v_{i}+e_{i j}, \quad i=1, \cdots, m, \quad j=1, \cdots, n_{i}
$$


where $g\left(p_{i}\right)$ is a function of $p_{i}, \delta$ is an unknown coefficient, $v_{i} \stackrel{i i d}{\sim}\left(0, \sigma_{v}^{2}\right)$ and $e_{i j} \stackrel{i i d}{\sim}\left(0, \sigma_{e}^{2}\right)$. The equation (3.17) can be written as follows:

$$
\mathbf{y}_{i}=\mathbf{A}_{i} \boldsymbol{\beta}^{\star}+\mathbf{1}_{n_{i}} v_{i}+\mathbf{e}_{i}, \quad i=1, \cdots, m,
$$

where $\mathbf{A}_{i}=\left(\mathbf{X}_{i} \mid g\left(p_{i}\right)\right)$ with $\mathbf{X}_{i}=\left(\mathbf{x}_{i 1}, \cdots, \mathbf{x}_{i n_{i}}\right)^{T}, \mathbf{y}_{i}=\left(y_{i 1}, \cdots, y_{i n_{i}}\right)^{T}$, $\mathbf{e}_{i}=\left(\mathbf{e}_{i 1}, \cdots, \mathbf{e}_{i n_{i}}\right)^{T}$ and $\boldsymbol{\beta}^{\star}=\left(\boldsymbol{\beta}, \delta_{0}\right)^{T}$.

The BLUP estimator of $\mu_{i}$ is:

$$
\tilde{\mu}_{i}=\overline{\mathbf{A}}_{i}^{T} \tilde{\boldsymbol{\beta}}^{\star}+\tilde{v}_{i}, \quad i=1, \cdots, m,
$$

where $\tilde{v}_{i}=\gamma_{i}\left(\bar{y}_{i}-\overline{\mathbf{a}}_{i}^{T} \tilde{\boldsymbol{\beta}}^{\star}\right)$ with $\overline{\mathbf{a}}_{i}=\sum_{j=1}^{n_{i}} \mathbf{a}_{i j} / n_{i}, \gamma_{i}=\frac{\sigma_{v}^{2}}{\sigma_{v}^{2}+\sigma_{e}^{2} / n_{i}}$ and $\tilde{\boldsymbol{\beta}}^{\star}=$ $\left(\sum_{i=1}^{m} \mathbf{A}_{i}^{T} \mathbf{V}_{i}^{-1} \mathbf{A}_{i}\right)^{-1}\left(\sum_{i=1}^{m} \mathbf{A}_{i}^{T} \mathbf{V}_{i}^{-1} \mathbf{y}_{i}\right)$, where $\mathbf{V}_{i}=\sigma_{v}^{2} \mathbf{1}_{n_{i}} \mathbf{1}_{n_{i}}^{T}+\sigma_{e}^{2} \mathbf{I}_{n_{i}}$. Variance components, $\sigma_{e}^{2}$ and $\sigma_{v}^{2}$ can be calculated by fixed point equations described in Section (3.2.1).

We obtain the EBLUP estimator of the area mean for sampled areas as follows:

$$
\hat{\mu}_{i}=\overline{\mathbf{A}}_{i}^{T} \hat{\boldsymbol{\beta}}^{\star}+\hat{v}_{i}, \quad i=1, \cdots, m,
$$

where $\hat{\boldsymbol{\beta}}$ and $\hat{v}_{i}$ are the values of $\tilde{\boldsymbol{\beta}}$ and $\tilde{v}_{i}$ in (3.18) when $\sigma_{v}^{2}$ and $\sigma_{e}^{2}$ are replaced by $\hat{\sigma}_{e}^{2}$ and $\hat{\sigma}_{v}^{2}$.

For non-sampled areas, we assume that the model holds for both sampled and non-sampled areas, and $p_{i}$ is known for non-sampled areas. For non-sampled 
areas, direct estimates are not available so we use a synthetic estimator:

$$
\hat{\mu}_{i}=\overline{\mathbf{A}}_{i}^{T} \hat{\boldsymbol{\beta}}^{\star}, \quad i=m+1, \cdots, M .
$$

We tested Verret et al.'s method with two choices of $g\left(p_{i}\right)$ :

$$
\begin{aligned}
& \text { - } g_{1}\left(p_{i}\right)=p_{i} \\
& \text { - } g_{2}\left(p_{i}\right)=\log \left(p_{i}\right)
\end{aligned}
$$

The following table presents the average absolute bias, $\overline{A B}$, and average root MSE, $\overline{R M S E}$, of the four predictors over $R=1000$ simulations, separately for sampled and non sampled areas. $\overline{A B}$ and $\overline{R M S E}$ are given in (3.15) and (3.16).

Table 3.2: Average absolute bias and average RMSE

\begin{tabular}{||cccccc||}
\hline$I_{i}$ & Performance measure & Ordinary & $p_{i}$ & $\log \left(p_{i}\right)$ & P-spline \\
\hline \hline 1 & $\overline{A B}$ & 0.011 & 0.007 & 0.004 & 0.003 \\
& $\overline{R M S E}$ & 0.394 & 0.5302 & 0.165 & 0.189 \\
\hline 0 & $\overline{A B}$ & 0.909 & 0.531 & 0.003 & 0.025 \\
& $\overline{R M S E}$ & 1.031 & 0.696 & 0.191 & 0.422 \\
\hline
\end{tabular}

Ignoring the informative sampling design results in large bias for both sampled and non-sampled areas. This large bias causes large RMSE's as well. Between the two choices for the augmented model, $\log \left(p_{i}\right)$ provides better results. This was expected as $b_{i}$ 's are having an exponential form. The P-spline results are comparable with $\log \left(p_{i}\right)$ in terms of bias, and are approximately unbiased. However the RMSE's values of P-spline technique are higher than the augmented model based on $\log \left(p_{i}\right)$. 
We computed the bootstrap MSE estimator for each $R=1000$ simulation run, using $B=200$ bootstrap samples. Let $\hat{M}_{\text {boot }}^{(r)}\left(\hat{\mu}_{i}\right)$ denote the bootstrap MSE estimate of $\hat{\mu}_{i}$ obtained from the $r^{\text {th }}$ simulated sample using (3.14). Let $M S E\left(\hat{\mu}_{i}\right)$ be the approximated true MSE obtained using $\mathrm{T}=10000$ simulated data, i.e.,

$$
\widehat{M S E}\left(\hat{\mu}_{i}\right)=\frac{1}{T} \sum_{t=1}^{T}\left(\hat{\mu}_{i}^{(t)}-\bar{Y}_{i}^{(t)}\right)^{2}
$$

where $\hat{\mu}_{i}^{(t)}$ and $\bar{Y}_{i}^{(t)}$ are $\hat{\mu}_{i}$ and $\bar{Y}_{i}$ based on the $t^{t h}$ simulated sample and population, respectively. Using the bootstrap estimates $\hat{M}_{\text {boot }}^{(r)}\left(\hat{\mu}_{i}\right)$, we estimate the mean of the MSE estimator by

$$
\widehat{E}\left\{M S E\left(\hat{\mu}_{i}\right)\right\}=\frac{1}{R} \sum_{r=1}^{R} \hat{M}_{\text {boot }}^{(r)}\left(\hat{\mu}_{i}\right) .
$$

For sampled areas, we estimate the average absolute relative bias (ARB) of $M S E\left(\hat{\mu}_{i}\right)$ as

$$
\overline{A R B}=\frac{1}{m} \sum_{i=1}^{m}\left|\frac{\hat{E}\left\{M S E\left(\hat{\mu}_{i}\right)\right\}}{\widehat{M S E}\left(\hat{\mu}_{i}\right)}-1\right| .
$$

For non-sampled areas,the $\overline{A R B}$ of the MSE estimator is calculated using

$$
\overline{A R B}=\frac{1}{M-m} \sum_{i=m+1}^{M}\left|\frac{\hat{E}\left\{M S E\left(\hat{\mu}_{i}\right)\right\}}{\widehat{M S E}\left(\hat{\mu}_{i}\right)}-1\right| .
$$

The following table reports the percent absolute relative bias $(\overline{\% A R B})$ of the MSE estimator, averaged over all areas. 
Table 3.3: $\overline{\% A R B}(\%)$ and MSE of bootstrap MSE estimator of EBLUP

\begin{tabular}{||cccccc||}
\hline$I_{i}$ & Performance measure & Ordinary & $p_{i}$ & $\log \left(p_{i}\right)$ & P-spline \\
\hline \hline 1 & $(\%) \overline{A R B}$ & 2.34 & 11.66 & 39.62 & 35.31 \\
& $M S E$ & 0.156 & 0.091 & 0.027 & 0.036 \\
\hline 0 & $(\%) \overline{A R B}$ & 66.67 & 72.23 & 42.06 & 42.01 \\
& $M S E$ & 1.079 & 0.484 & 0.036 & 0.177 \\
\hline
\end{tabular}

For sampled areas, the augmented model with $\log \left(p_{i}\right)$ exhibits the largest $\overline{A R B}$ $(39.6 \%)$ followed by the P-spline model with $\overline{A R B}$ of $35 \%$. Although the MSE of the ordinary EBLUP is large, its $\overline{A R B}$ value is small (2.3\%). Overall, the bootstrap MSE of non-sampled areas does not perform very well and leads to large $\overline{A R B}(>40 \%)$. In particular, the choice of $p_{i}$ has the largest $\overline{A R B}(72.2 \%)$.

\subsection{Summary}

In continuation of the previous chapter, we studied unit level models under twostage informative sampling, and proposed a semi-parametric approach to address the bias due to informativeness of the sampling design. We studied three possible cases depending on the sampling design of each stage and discussed different scenarios according to the availability of selection probabilities in non-sampled areas. Similar to one-stage sampling, we proposed a conditional bootstrap method for estimating the MSE, which requires the availability of $p_{i}$ and $p_{j \mid i}$ for all units in the population.

A simulation study was conducted under two scenarios: 1) Case I, where both stages of sampling are informative and 2) Case II, where only the first stage of sam- 
pling is informative. For Case I, we compared the results with the bias-adjusted estimator of Pfeffermann and Sverchkov (PS). In terms of bias, PS method performed better, however the P-spline method exhibited lower MSE. It should be noted that PS model assumes the knowledge of models for the sampling weights, unlike the proposed method. We also examined the performance of the P-spline method using linear and quadratic splines. Under the considered simulation setting, the quadratic spline performed better. For Case II, the results are compared to the augmented method proposed by Verret et al. (2015). For sampled areas, the P-spline approach performs very well in term of bias and its MSE is comparable to the augmented model. For non-sampled areas, the RMSE of the P-spline approach is lower than the other method, but its bias is larger than the corresponding bias of the augmented models with the choice of $\log \left(p_{i}\right)$. 


\section{Chapter 4}

\section{EB estimation under unmatched two-fold subarea models}

In small area estimation, an area-level model can be divided into two parts: a sampling model and a linking model. The area-level model assumes that a suitable function of a small area parameter of interest is related to area specific auxiliary information through a linking model. A direct estimator of a small area parameter is not accurate when the sample size of an area is small. Area specific auxiliary information can be used to improve estimation accuracy of the small area parameter if a suitable model between the parameter and the auxiliary variable (linking model) can be found. In the basic area-level model, a linear link between the parameter and the auxiliary variable is used. However, when the actual link is non-linear, applying the basic area-level model will produce erroneous inference, leading to biased and inefficient small area estimators. The proper model, in this case, should use a properly specified nonlinear link function, and such a model is called an unmatched area-level model. The unmatched one-fold area-level models 
have been studied in the literature (You and Rao (2002) and Sugasawa, Kubokawa and Rao (2018). In this chapter, we study estimation of subarea means under two-fold unmatched sampling and linking models, using an empirical best (EB) approach.

We first review the unmatched one-fold area-level model and the matched twofold area-level model in Section 4.1 and 4.2. The proposed EB method is discussed in Section 4.3, followed by a parametric bootstrap method for estimating the mean squared error (MSE) in Section 4.4. We extend the proposed method to a threefold model in Section 4.5. Results of a simulation study are reported in Section 4.6. A brief summary of the chapter is given in Section 4.7.

\subsection{Unmatched one-fold model}

The basic area-level model has two main parts: linking model and sampling model. The linking model assumes that some suitable function of the small area parameter of interest, $h\left(\theta_{i}\right)$, is related to auxiliary information through a linear model.

$$
h\left(\theta_{i}\right)=\mathbf{x}_{i}^{T} \boldsymbol{\beta}+v_{i}, \quad i=1, \cdots, m,
$$

where $m$ is the number of sampled areas, $\boldsymbol{\beta}$ is a $p \times 1$ vector of regression parameters and $v_{i} \stackrel{i i d}{\sim} N\left(0, \sigma_{v}^{2}\right)$. On the other hand, the sampling model assumes that a direct estimator of $\theta_{i}, \hat{\theta}_{i}$, is available:

$$
\hat{\theta}_{i}=\theta_{i}+e_{i}
$$

where $e_{i} \stackrel{i n d}{\sim} N\left(0, \psi_{i}\right)$ with known $\psi_{i}$, and $e_{i}$ is independent of $v_{i}$. 
A common area-level model used for the estimation of small area means is the Fay-Herriot model (Fay and Herriot, 1979), in which $h\left(\theta_{i}\right)=\theta_{i}$. Since $h(\cdot)$ is an identity link, this model is also called a matched area-level model. The Fay-Herriot model is obtained by combining the linking and the sampling models resulting in the one-fold area-level model:

$$
\hat{\theta}_{i}=\mathbf{x}_{i}^{T} \boldsymbol{\beta}+v_{i}+e_{i} \quad i=1, \ldots, m .
$$

When $h(\cdot)$ is a non-linear function, the sampling and linking models are called unmatched. Estimation of a small area parameter $\theta_{i}$ under an unmatched arealevel model is significantly more complicated than that under the Fay-Herriot model. You and Rao (2002) used a hierarchical Bayes (HB) approach to deal with unmatched sampling and linking models for small area estimation. Sugasawa et al. (2018) studied EB estimation under unmatched area level models.

As mentioned earlier, in this chapter, we study unmatched two-fold subarea model. The goal is to use an EB approach for the estimation of subarea means, $\theta_{i j}$. Before discussing the proposed method, we review the matched two fold model in the following section.

\subsection{Matched two-fold subarea model}

Torabi and Rao (2014) proposed an extension of the Fay and Herriot (1979) arealevel model to the subarea level, where the target population is divided into M areas (e.g. states) and each area $i$ is also divided into $N_{i}$ subareas (e.g. counties). The parameters of interest are the area mean, $\theta_{i}$, and the subarea mean, $\theta_{i j}$. The 
linking model of the two-fold subarea model is given by

$$
\theta_{i j}=\mathbf{x}_{i j}^{T} \boldsymbol{\beta}+v_{i}+u_{i j}, \quad i=1, \cdots, M, \quad j=1, \cdots, N_{i}
$$

where $\mathbf{x}_{i j}$ is a $p \times 1$ vector of subarea level auxiliary variables, $\boldsymbol{\beta}$ is the vector of regression parameter, $v_{i} \stackrel{i i d}{\sim} N\left(0, \sigma_{v}^{2}\right)$ is the area effect and $u_{i j} \stackrel{i i d}{\sim} N\left(0, \sigma_{u}^{2}\right)$ is the subarea effect, which is independent of $v_{i}$.

We assume that $n_{i}$ subareas are sampled from $N_{i}$ subareas in area $i$. The sampling model is given by

$$
\hat{\theta}_{i j}=\theta_{i j}+e_{i j}
$$

where $e_{i j} \stackrel{i n d}{\sim} N\left(0, \psi_{i j}\right)$, with known $\psi_{i j}$. Combining (4.1) and (4.2), we will get the two-fold subarea-level model:

$$
\hat{\theta}_{i j}=\mathbf{x}_{i j}^{T} \boldsymbol{\beta}+v_{i}+u_{i j}+e_{i j}, \quad j=1, \ldots, n_{i}, \quad i=1, \ldots, m
$$

The above equation is a special case of a general linear mixed model with block diagonal covariance structure. Let $\mathbf{y}_{i}=\left(\hat{\theta}_{i 1}, \cdots, \hat{\theta}_{i n_{i}}\right)^{T}$ and $\mathbf{e}_{i}=\left(e_{i 1}, \cdots, e_{i n_{i}}\right)^{T}$, (4.3) can be written as:

$$
\mathbf{y}_{i}=\mathbf{X}_{i} \boldsymbol{\beta}+\mathbf{Z}_{i} \mathbf{v}_{i}+\mathbf{e}_{i}, \quad i=1, \ldots, m,
$$

where $\mathbf{X}_{i}$ is the $n_{i} \times p$ matrix with rows $\mathbf{x}_{i j}^{T}, \mathbf{Z}_{i}=\left(\mathbf{1}_{n i} \mid \mathbf{I}_{n i}\right)$, and $\mathbf{v}_{i}=\left(v_{i}, \mathbf{u}_{i}^{T}\right)^{T}$ with $\mathbf{u}_{i}=\left(u_{i 1}, \cdots, u_{i, n_{i}}\right)^{T}$. The variance-covariance matrix of $\mathbf{y}_{i}$ has the following form:

$$
\mathbf{V}_{i}=\sigma_{v}^{2} \mathbf{1}_{n_{i}} \mathbf{1}_{n_{i}}^{T}+\operatorname{diag}\left(\sigma_{u}^{2}+\psi_{i 1}+\cdots, \sigma_{u}^{2}+\psi_{i n_{i}}\right)
$$


Under this setup, for a given $\boldsymbol{\delta}=\left(\sigma_{v}^{2}, \sigma_{u}^{2}\right)^{T}$, the best linear unbiased predictor (BLUP) estimator of $v_{i}$ and $u_{i j}$ are given by:

$$
\begin{gathered}
\tilde{v}_{i}(\boldsymbol{\delta})=\gamma_{i}\left[\hat{\theta}_{i \gamma}-\overline{\mathbf{x}}_{i \gamma}^{T} \tilde{\boldsymbol{\beta}}(\boldsymbol{\delta})\right] \\
\tilde{u}_{i j}(\boldsymbol{\delta})=\gamma_{i j}\left[\hat{\theta}_{i j}-\mathbf{x}_{i j}^{T} \tilde{\boldsymbol{\beta}}(\boldsymbol{\delta})\right]-\gamma_{i} \gamma_{i j}\left[\hat{\theta}_{i \gamma}-\overline{\mathbf{x}}_{i \gamma}^{T} \tilde{\boldsymbol{\beta}}(\boldsymbol{\delta})\right],
\end{gathered}
$$

where $\tilde{\boldsymbol{\beta}}(\boldsymbol{\delta})$ is the weighted least squares (WLS) estimator of $\boldsymbol{\beta}, \gamma_{i j}=\sigma_{u}^{2} /\left(\sigma_{u}^{2}+\psi_{i j}\right)$, $\gamma_{i}=\sigma_{v}^{2} /\left(\sigma_{v}^{2}+\sigma_{u}^{2} / \gamma_{i .}\right), \hat{\theta}_{i \gamma}=\gamma_{i .}^{-1} \sum_{j=1}^{n_{i}} \hat{\theta}_{i j}, \overline{\mathbf{x}}_{i \gamma}=\gamma_{i .}{ }^{-1} \sum_{j=1}^{n_{i}} \mathbf{x}_{i j}$, and $\gamma_{i .}=\sum_{j=1}^{n_{i}} \gamma_{i j}$.

The BLUP estimator of $\theta_{i j}$ for a sampled subarea is:

$$
\tilde{\theta}_{i j}(\boldsymbol{\delta})=\mathbf{x}_{i j}^{T} \tilde{\boldsymbol{\beta}}(\boldsymbol{\delta})+\tilde{v}_{i}(\boldsymbol{\delta})+\tilde{u}_{i j}(\boldsymbol{\delta}), \quad j=1, \cdots, n_{i}, \quad i=1, . \cdots, m,
$$

where $\tilde{v}_{i}(\boldsymbol{\delta})$ and $\tilde{u}_{i j}(\boldsymbol{\delta})$ are given by (4.5) and (4.6). Using the quasi-BLUP likelihood estimator, for a non-sampled subarea we have:

$$
\tilde{\theta}_{i k}(\boldsymbol{\delta})=\mathbf{x}_{i k}^{T} \tilde{\boldsymbol{\beta}}(\boldsymbol{\delta})+\tilde{v}_{i}(\boldsymbol{\delta}), \quad k=n_{i}+1, \cdots, N_{i}, \quad i=1, \ldots, m
$$

The BLUP estimator of the area mean $\theta_{i}$ is

$$
\tilde{\theta}_{i}(\boldsymbol{\delta})=N_{i .}^{-1}\left[\sum_{j=1}^{n_{i}} N_{i j} \tilde{\theta}_{i j}(\boldsymbol{\delta})+\sum_{k=n_{i}+1}^{N_{i}} N_{i k} \tilde{\theta}_{i k}(\boldsymbol{\delta})\right]
$$

where $N_{i .}=\sum_{j=1}^{N_{i}} N_{i j}$. Replacing $\boldsymbol{\delta}$ with $\hat{\boldsymbol{\delta}}=\left(\hat{\sigma}_{v}^{2}, \hat{\sigma}_{u}^{2}\right)$ we can obtain the EBLUP of the area and subarea means. 


\subsection{Unmatched two-fold model}

The EBLUP estimator discussed in Section 4.2 is under matched linking and sampling models. As the case of unmatched one-fold model, when the relationship between $\theta_{i j}$ and covariate $\mathbf{x}_{i j}$ cannot be characterized by a linking model with an identity link, naively applying a matched two-fold model may lead to incorrect inference and inefficient estimators. In this case, an unmatched model is needed.

Mohadjer et al. (2012) studied a hierarchical Bayes (HB) approach under the unmatched two-fold model with logit link for adult literacy data. We propose an EB approach to estimate small subarea means under an unmatched two-fold subarea level models.

Let the sampling model be:

$$
y_{i j}=\theta_{i j}+e_{i j}, \quad i=1, \ldots, m \quad j=1, \ldots, n_{i}
$$

where $j$ denotes a sampled subarea within sampled area $i, \theta_{i j}$ represents the subarea parameter of interest, $y_{i j}$ is a direct estimator of $\theta_{i j}$ for sampled subareas and $e_{i j}$ 's are the sampling error with $e_{i j} \stackrel{i n d}{\sim} N\left(0, \psi_{i j}\right)$. We assume that $\psi_{i j}$ 's are known.

On the other hand, the linking model is given by:

$$
h\left(\theta_{i j}\right)=\mathbf{x}_{i j}^{T} \boldsymbol{\beta}+v_{i}+u_{i j}, \quad i=1, \ldots, M \quad j=1, \ldots, N_{i},
$$

where $u_{i j}$ is the subarea-level random effect, $v_{i}$ is the area-level random effect and $h(\cdot)$ denotes a monotonic function. For example, it may be chosen as $h(t)=\log (t)$ or $h(t)=\operatorname{logit}(t)=\log \left(\frac{t}{1-t}\right)$, depending on the situation. We further assume that: 
- $v_{i} \stackrel{i i d}{\sim} f\left(v ; \boldsymbol{\eta}_{v}\right)$, where $\boldsymbol{\eta}_{v}$ is a parameter and $f$ is a generic notation for density,

- $u_{i j} \stackrel{i i d}{\sim} f\left(u ; \boldsymbol{\eta}_{u}\right)$, where $\boldsymbol{\eta}_{u}$ is a parameter and $f$ is a generic notation for density,

- $v_{i}, u_{i j}, e_{i j}$ are mutually independent.

Our goal is to estimate the subarea means, $\theta_{i j}$. However, as mentioned above, the sampling model and the linking model (4.8) and (4.9) cannot be combined as $h(\cdot)$ is not a linear function. Moreover, unlike the one-fold model, where data are all independent, $y_{i j}$ 's are correlated within each sampled area $i$ due to the presence of $v_{i}$. We obtain the EB estimator of the $\theta_{i j}$ in three main steps:

1. Obtain the best predictor (BP) of $\theta_{i j}$, assuming that the model parameters, $\boldsymbol{\eta}=\left(\boldsymbol{\beta}, \boldsymbol{\eta}_{v}, \boldsymbol{\eta}_{u}\right)$, are known. The BP is given by

$$
\tilde{\theta}_{i j}(\boldsymbol{\eta})=E\left(\theta_{i j} \mid \mathbf{y} ; \boldsymbol{\eta}\right) \quad i=1, \cdots, M, \quad j=1, \cdots, N_{i},
$$

where $\mathbf{y}=\left\{y_{i j}: i=1, \cdots, m, \quad j=1, \cdots, n_{i}\right\}$.

2. Obtain the maximum likelihood estimator (MLE) of $\boldsymbol{\eta}$, denoted by $\hat{\boldsymbol{\eta}}$.

3. Replace $\boldsymbol{\eta}$ with $\hat{\boldsymbol{\eta}}$ in (4.10) to get the EB estimation of $\theta_{i j}$, i.e.,

$$
\hat{\theta}_{i j}=\tilde{\theta}_{i j}(\hat{\boldsymbol{\eta}}) .
$$

We will discuss these steps in the next subsections. 


\subsubsection{BP estimators of subarea parameters $\theta_{i j}$}

Assuming that the model parameters $\boldsymbol{\eta}=\left(\boldsymbol{\beta}, \boldsymbol{\eta}_{v}, \boldsymbol{\eta}_{u}\right)$ are known, we find the BP estimator of $\theta_{i j}$, which is given by the conditional expectation of $\theta_{i j}$ given $\mathbf{y}=$ $\left\{y_{i j}, i=1, \cdots, m, j=1, \cdots, n_{j}\right\}$. Let $\mathbf{y}_{i}=\left(y_{i 1}, y_{i 2}, \cdots, y_{i n_{i}}\right)^{T}$ be a $n_{i} \times 1$ vector and $\mathbf{u}_{i}=\left(u_{i 1}, u_{i 2}, \cdots, u_{i n_{i}}\right)^{T}$. From $(4.9), \theta_{i j}$ can be considered as a function of $u_{i j}$ and $v_{i}$ :

$$
\theta_{i j}=h^{-1}\left(\mathbf{x}_{i j}^{T} \boldsymbol{\beta}+v_{i}+u_{i j}\right)
$$

Hence, $E\left(\theta_{i j} \mid \mathbf{y} ; \boldsymbol{\eta}\right)$ is given by

$$
\begin{aligned}
\tilde{\theta}_{i j}(\boldsymbol{\eta}) & =\int \theta_{i j} f\left(v_{i}, u_{i j} \mid \mathbf{y}_{i} ; \boldsymbol{\eta}\right) d v_{i} d u_{i j} \\
& =\int \theta_{i j} f\left(v_{i}, \mathbf{u}_{i} \mid \mathbf{y}_{i} ; \boldsymbol{\eta}\right) d v_{i} d \mathbf{u}_{i}
\end{aligned}
$$

where " $f$ " denotes a density function in general.

In equation (4.11), the second equality has some computational advantages over the first one. The following makes this more clear:

$$
\begin{aligned}
f\left(v_{i}, \mathbf{u}_{i}, \mathbf{y}_{i}\right) & =f\left(\mathbf{u}_{i}, \mathbf{y}_{i} \mid v_{i}\right) f\left(v_{i}\right) \\
& =\left\{\prod_{j=1}^{n_{i}} f\left(y_{i j}, u_{i j} \mid v_{i}\right)\right\} f\left(v_{i}\right) \\
& =f\left(v_{i}\right) \prod_{j=1}^{n_{i}}\left\{f\left(y_{i j} \mid u_{i j}, v_{i}\right) f\left(u_{i j} \mid f\left(v_{i}\right)\right\}\right. \\
& =f\left(v_{i}\right) \prod_{j=1}^{n_{i}}\left\{f\left(y_{i j} \mid u_{i j}, v_{i}\right) f\left(u_{i j}\right)\right\}
\end{aligned}
$$

Hence, $f\left(v_{i}, \mathbf{u}_{i} \mid \mathbf{y}_{i}\right) \propto f\left(v_{i}\right)\left\{\prod_{j=1}^{n_{i}} f\left(y_{i j} \mid u_{i j}, v_{i}\right) f\left(u_{i j}\right)\right\}$, which means $\left(v_{i}, u_{i j}\right)$ are not conditionally independent of $u_{i l}, l \neq j$, given $\mathbf{y}_{i}$, due to the presence of 
$f\left(y_{i j} \mid u_{i j}, v_{i}\right)$. As a result, $f\left(v_{i}, u_{i j} \mid \mathbf{y}_{i}\right)$ does not have a simple analytical form. Therefore, we use $f\left(v_{i}, \mathbf{u}_{i} \mid \mathbf{y}_{i}\right)$ instead to avoid this complication.

Depending on the values of $i$ and $j$, we may have three possible cases:

- Case I: sampled subarea within sampled area

- Case II: non-sampled subarea within sampled area

- Case III: non-sampled subarea within non-sampled area

Figure (4.1) displays the three possible cases for $M=6$ areas and $N_{1}=N_{2}=$ $\ldots=N_{6}=5$ subareas. The yellow areas represent the two sampled areas (Area 3 and Area 6) and the blue parts show the sampled subareas.

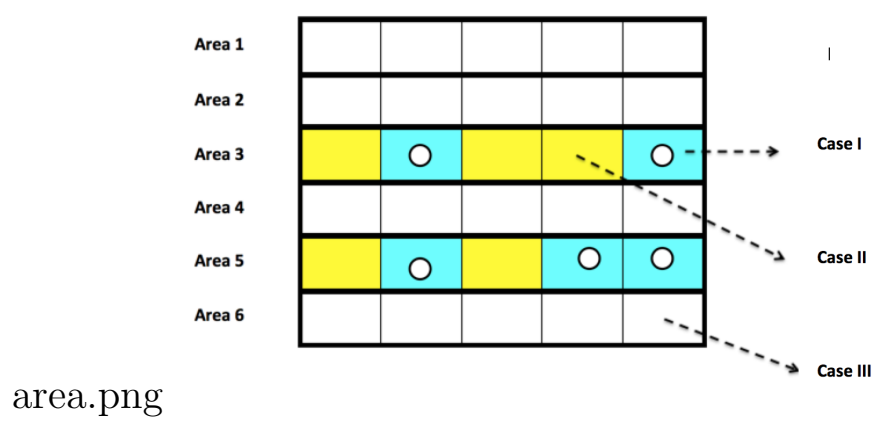

Figure 4.1: Two-fold subarea model: representation of areas and subareas

The derivation of $\mathrm{BP}$ of subarea parameter $\theta_{i j}$ for each case is discussed below.

\section{Case I: sampled subarea within sampled area}

In the case of sampled areas $i=1, \cdots, m$ and sampled subareas $j=1, \cdots, n_{i}$, we obtain $f\left(\mathbf{y}_{i}\right)$ by integrating with respect to $v_{i}$ and $\mathbf{u}_{i}$. 
Going back to (4.11), we have:

$$
\begin{aligned}
\tilde{\theta}_{i j}(\boldsymbol{\eta}) & =\int \theta_{i j} f\left(v_{i}, \mathbf{u}_{i} \mid \mathbf{y}_{i} ; \boldsymbol{\eta}\right) d v_{i} d \mathbf{u}_{i} \\
& =\int \theta_{i j} \frac{f\left(v_{i}, \mathbf{u}_{i}, \mathbf{y}_{i} ; \boldsymbol{\eta}\right)}{f\left(\mathbf{y}_{i}\right)} d v_{i} d \mathbf{u}_{i} \\
& =\frac{\int \theta_{i j} f\left(v_{i}, \mathbf{u}_{i}, \mathbf{y}_{i} ; \boldsymbol{\eta}\right) d v_{i} d \mathbf{u}_{i}}{f\left(\mathbf{y}_{i}\right)} \\
& =\frac{\int \theta_{i j} f\left(v_{i}, \mathbf{u}_{i}, \mathbf{y}_{i} ; \boldsymbol{\eta}\right) d v_{i} d \mathbf{u}_{i}}{\int f\left(v_{i}, \mathbf{u}_{i}, \mathbf{y}_{i}\right) d v_{i} d \mathbf{u}_{i}}
\end{aligned}
$$

Given $\boldsymbol{\eta}$, for some measurable function $g(\cdot, \cdot)$, we should compute:

$$
\begin{aligned}
G(\boldsymbol{\eta}) & :=\int g\left(v_{i}, \mathbf{u}_{i}\right) f\left(v_{i}, \mathbf{u}_{i}, \mathbf{y}_{i} ; \boldsymbol{\eta}\right) d v_{i} d \mathbf{u}_{i} \\
& =\int g\left(v_{i}, \mathbf{u}_{i}\right) \frac{f\left(v_{i}, \mathbf{u}_{i}, \mathbf{y}_{i} ; \boldsymbol{\eta}\right)}{f\left(v_{i}, \mathbf{u}_{i} ; \boldsymbol{\eta}\right)} f\left(v_{i}, \mathbf{u}_{i} ; \boldsymbol{\eta}\right) d v_{i} d \mathbf{u}_{i} \\
& =\int\left\{g\left(v_{i}, \mathbf{u}_{i}\right) f\left(\mathbf{y}_{i} \mid v_{i}, \mathbf{u}_{i} ; \boldsymbol{\eta}\right)\right\} f\left(v_{i}, \mathbf{u}_{i} ; \boldsymbol{\eta}\right) d v_{i} d \mathbf{u}_{i}
\end{aligned}
$$

The above expression can be computed via Monte Carlo integration. We review the Monte Carlo integration technique below.

\section{Monte Carlo integration}

Monte Carlo (MC) integration is a numerical method for computing the value of integrals using probabilistic techniques. The objective is to estimate an integral of the form

$$
I=\int g(x) f(x) d x
$$


where $g$ is a function and $f$ is a probability density function (PDF) of a random variable $X$. Equation (4.15) can be seen as the expected value of $g(x)$ :

$$
\int g(x) f(x) d x=E(g(X))=I .
$$

By the law of large numbers, one way to approximate the above integration is to generate a large number of (say $T$ ) of random variables $X_{i}, i=1, \cdots, T$ from a distribution with PDF $f(x)$, and use the sample average

$$
\hat{I}=\frac{1}{T} \sum_{i=1}^{T} f\left(X_{i}\right) .
$$

Going back to (4.14), we generate $T$ random vector values $\left(v_{i, t}^{\star}, \mathbf{u}_{i, t}^{\star}\right)$, for $t=$ $1, \cdots, T$ from the distribution of $\left(v_{i}, \mathbf{u}_{i}\right)$ given $\boldsymbol{\eta}$. The $G(\boldsymbol{\eta})$ can be approximated by

$$
G(\boldsymbol{\eta}) \approx \frac{1}{T} \sum_{t=1}^{T} g\left(v_{i, t}^{\star}, \mathbf{u}_{i, t}^{\star}\right) f\left(\mathbf{y}_{i} \mid v_{i, t}^{\star}, \mathbf{u}_{i, t}^{\star} ; \boldsymbol{\eta}\right)
$$

\section{Case II: non-sampled subarea within sampled area}

In the case of sampled areas $i=1, \cdots, m$ and non-sampled subareas $l=\left(n_{i}+\right.$ 1), $\cdots, N_{i}$, where $l \neq j, u_{i l}$ is independent of $\left(\mathbf{y}_{i}, v_{i}, \mathbf{u}_{i}\right)$. So, we will have:

$$
\begin{aligned}
f\left(v_{i}, \mathbf{u}_{i}, u_{i l} \mid \mathbf{y}\right) & =f\left(v_{i}, \mathbf{u}_{i} \mid \mathbf{y}\right) f\left(u_{i l}\right) \\
& =f\left(v_{i}, \mathbf{u}_{i} \mid \mathbf{y}_{i}\right) f\left(u_{i l}\right) \\
& =\frac{f\left(v_{i}, \mathbf{u}_{i}, \mathbf{y}_{i}\right) f\left(u_{i l}\right)}{f\left(\mathbf{y}_{i}\right)}
\end{aligned}
$$


Hence, $\tilde{\theta}_{i l}(\boldsymbol{\eta})$ can be calculated as follows

$$
\begin{aligned}
\tilde{\theta}_{i l}(\boldsymbol{\eta}) & =\int \theta_{i l} f\left(v_{i}, u_{i l} \mid \mathbf{y}_{i} ; \boldsymbol{\eta}\right) d v_{i} d u_{i l} \\
& =\int \theta_{i l} f\left(v_{i}, \mathbf{u}_{i}, u_{i l} \mid \mathbf{y}_{i} ; \boldsymbol{\eta}\right) d v_{i} d \mathbf{u}_{i} d u_{i l} \\
& =\frac{\int \theta_{i l} f\left(v_{i}, \mathbf{u}_{i}, \mathbf{y}_{i} ; \boldsymbol{\eta}\right) f\left(u_{i l} ; \boldsymbol{\eta}\right) d v_{i} d \mathbf{u}_{i} d u_{i l}}{\int f\left(\mathbf{y}_{i}, v_{i}, \mathbf{u}_{i} ; \boldsymbol{\eta}\right) d v_{i} d \mathbf{u}_{i}}
\end{aligned}
$$

The presence of $v_{i}$ helps the estimation of $\theta_{i l}$ by pooling information from observed

$\mathbf{y}_{i}$. Similar to the case I, the above integration can be computed through MC integration.

\section{Case III: non-sampled subarea within non-sampled area}

For non-sampled area $k$, where $k=(m+1), \cdots, M, l=1, \cdots, N_{k},\left(v_{k}, u_{k l}\right)$ is independent of $\mathbf{y}$.

$$
f\left(v_{k}, u_{k l} \mid \mathbf{y}\right)=f\left(v_{k}, u_{k l}\right)=f\left(v_{k}\right) f\left(u_{k l}\right)
$$

The BP $\tilde{\theta}_{k l}$ can be obtained as follows:

$$
\begin{aligned}
\tilde{\theta}_{k l}(\boldsymbol{\eta}) & =\int \theta_{k l} f\left(v_{k}, u_{k l} \mid \mathbf{y}_{i} ; \boldsymbol{\eta}\right) d v_{k} d_{u k l} \\
& =\int \theta_{k l} f\left(v_{k} ; \boldsymbol{\eta}\right) f\left(u_{k l} ; \boldsymbol{\eta}\right) d v_{k l} d u_{k l} .
\end{aligned}
$$

For a given $\boldsymbol{\eta}$, data $\mathbf{y}$ does not play a role in the estimation of $\theta_{k l}$. Hence, in this case, $\tilde{\theta}_{k l}(\boldsymbol{\eta})$ is a synthetic estimator of $\theta_{k l}$ which primarily use the information in covariates $\mathbf{x}_{i j}$. Similar to previous cases, MC integration can be used for computing the $\tilde{\theta}_{k l}(\boldsymbol{\eta})$. 


\subsubsection{Estimation of model parameters}

After obtaining the BP, we should estimate the model parameters $\boldsymbol{\eta}$. We use a maximum likelihood estimator (MLE) to estimate the model parameters. For $\mathbf{y}_{i}$, $i=1, \cdots, m$ the likelihood function is defined by

$$
L(\boldsymbol{\eta} \mid \mathbf{y})=\prod_{i=1}^{m} f\left(\mathbf{y}_{i} ; \boldsymbol{\eta}\right)
$$

with

$$
f\left(\mathbf{y}_{i} ; \boldsymbol{\eta}\right)=\int f\left(v_{i}, \mathbf{u}_{i}, \mathbf{y}_{i} ; \boldsymbol{\eta}\right) d v_{i} d \mathbf{u}_{i} \approx \frac{1}{T} \sum_{t=1}^{T} f\left(\mathbf{y}_{i} \mid v_{i, t}^{\star}, \mathbf{u}_{i, t}^{\star} ; \boldsymbol{\eta}\right)
$$

where $\left(v_{i, t}^{\star}, \mathbf{u}_{i, t}^{\star}\right)$ are generated iid from $f\left(v_{i}, \mathbf{u}_{i} ; \boldsymbol{\eta}\right)$.

Each function evaluation requires a $\mathrm{MC}$ integration. One option is to use a general-purpose optimization method to directly maximize $L(\boldsymbol{\eta})$. Another option is to use the Expectation-Maximization (EM) algorithm.

\section{EM algorithm}

Dempster et al. (1977) proposed the Expectation-Maximization (EM) algorithm for maximizing the likelihood function. The EM algorithm is mainly used in two occasions:

1. When data is incomplete or it has missing values due to some collection or observation issues,

2. When optimizing the likelihood function is analytically difficult, but it can be simplified if we assume the existence of additional but missing parameters.

Let $X_{1}, \cdots, X_{n}$ be a set of observed data with joint density $f(\mathbf{x} ; \boldsymbol{\theta})$, where $\boldsymbol{\theta}$ is the unknown parameter. Assuming that $Y_{1}, \cdots, Y_{m}$ is a set of unobserved 
random variable with a distribution that also depends on $\boldsymbol{\theta}$, we can denote the joint density of $X_{1}, \cdots, X_{n}$ and $Y_{1}, \cdots, Y_{m}$ as $h(\mathbf{x}, \mathbf{y} ; \boldsymbol{\theta})$ and represent $g(\mathbf{y} \mid \mathbf{x} ; \boldsymbol{\theta})$ as the conditional density of $\mathbf{y}$ given $\mathbf{x}$.

The likelihood function of $\boldsymbol{\theta}$ is $L(\boldsymbol{\theta})=f(\mathbf{x} ; \boldsymbol{\theta})$. The goal is to find $\hat{\theta}_{M L E}$ :

$$
\hat{\theta}_{M L E}=\underset{\theta}{\arg \max } L(\boldsymbol{\theta})=\underset{\boldsymbol{\theta}}{\arg \max } f(\mathbf{x} ; \boldsymbol{\theta}) .
$$

If $f(\mathbf{x} ; \boldsymbol{\theta})$ has a complicated form, then we use $h(\mathbf{x}, \mathbf{y} ; \boldsymbol{\theta})$ and $g(\mathbf{y} \mid \mathbf{x} ; \boldsymbol{\theta})$ to simplify the computation. More precisely, let $L^{c}(\boldsymbol{\theta})=h(\mathbf{x}, \mathbf{y} ; \boldsymbol{\theta})$ be the complete likelihood. For an arbitrary parameter value $\boldsymbol{\theta}^{\star}$ we define the objective function $Q_{\boldsymbol{\theta}^{\star}}(\boldsymbol{\theta})$ as below:

$$
\begin{aligned}
Q_{\boldsymbol{\theta}^{\star}}(\boldsymbol{\theta}) & =E_{\boldsymbol{\theta}^{\star}}\left[\log L^{c}(\boldsymbol{\theta}) \mid \mathbf{x}\right] \\
& =E_{\boldsymbol{\theta}^{\star}}[\log h(\mathbf{x}, \mathbf{y} ; \boldsymbol{\theta}) \mid \mathbf{x}] \\
& =\int \log \{h(\mathbf{x}, \mathbf{y} ; \boldsymbol{\theta})\} g\left(\mathbf{y} \mid \mathbf{x} ; \boldsymbol{\theta}^{\star}\right) d y,
\end{aligned}
$$

where $E_{\boldsymbol{\theta}^{\star}}(. \mid \mathbf{x})$ is the conditional expectation with respect to $g\left(\mathbf{y} \mid \mathbf{x} ; \boldsymbol{\theta}^{\star}\right)$.

The EM algorithm is an iterative process of maximizing $Q_{\boldsymbol{\theta}^{\star}}(\boldsymbol{\theta})$, and it is usually described in two steps (E-step and the M-step). The process starts with an initial value $\theta^{(0)}$. We repeat the algorithm until we reach convergence. Assuming that $\theta^{(k)}$ is the values obtained at step $k$, we compute $\theta^{(k+1)}$ as follows:

1. E-step

Obtain $Q_{\hat{\theta}^{(k)}}(\theta)=E_{\hat{\theta}^{(k)}}\left\{\log L^{c}(\boldsymbol{\theta}) \mid \mathbf{x}\right\}$

2. M-step

Compute $\hat{\theta}^{(k+1)}=\arg \max _{\theta} Q_{\hat{\theta}^{(k)}}(\theta)$. 
We repeat the above steps until a convergence rule is reached.

Going back to the estimation of parameters $\boldsymbol{\eta}$ under the two-fold model, we treat $\left(v_{i} ; \mathbf{u}_{i}\right), i=1, \cdots, m$ as unobserved data. The log-complete likelihood is given by

$$
\log L_{c}(\boldsymbol{\eta})=\sum_{i=1}^{m} \log f\left(\mathbf{y}_{i}, v_{i}, \mathbf{u}_{i} ; \boldsymbol{\eta}\right)
$$

The Q-function in EM algorithm is

$$
Q^{(k)}(\boldsymbol{\eta})=\sum_{i=1}^{m} \int\left\{\log f\left(\mathbf{y}_{i}, v_{i}, \mathbf{u}_{i} ; \boldsymbol{\eta}\right)\right\} f\left(v_{i}, \mathbf{u}_{i} \mid \mathbf{y}_{i} ; \boldsymbol{\eta}^{(k)}\right) d v_{i} d \mathbf{u}_{i}
$$

The evaluation of $Q(\boldsymbol{\eta})$ can be done with MC integration, as in (4.14).

\section{General-purpose optimization}

The maximization of the log-likelihood function $\log L(\theta)$ can also be carried out using a general-purpose optimization algorithm such as the Newton-Raphson method or a quasi-Newton method. However, Newton-type methods require the first-order derivatives of the log-likelihood function to be evaluated, which also needs MC integration and will greatly increase computation burden. Hence, in this case, gradient-free optimization methods will hold an advantage in terms of computational efficiency.

We use a gradient-free simplex optimization method provided by the $\mathrm{R}$ package nlopt to obtain the MLEs. 


\subsubsection{EB estimator}

Replacing $\boldsymbol{\eta}$ in (4.10) with the MLE $\hat{\boldsymbol{\eta}}$, which is derived through EM algorithm or any other numerical methods, we can obtain the EB estimator of $\theta_{i j}$ :

$$
\hat{\theta}_{i j}=\tilde{\theta}_{i j}(\hat{\boldsymbol{\eta}})
$$

We consider two special cases: 1) unmatched model with normally distributed $v_{i}$ and $u_{i j}$, and 2) matched case (i.e., $h(t)=t$ ) with normally distributed $v_{i}$ and $u_{i j}$ in the next subsections.

\subsubsection{Special case I: normal random effects}

The estimation process described in the previous subsections does not need the normality assumption and can be used for any underlying distribution. However, under normality the conditional density functions have a familiar form and numerical computations are less complicated.

Under normality assumption, $\epsilon_{i j}, v_{i}$ and $u_{i j}$ in (4.8) and (4.9) are all normally distributed:

$$
\begin{aligned}
& f\left(v_{i}\right)=\frac{1}{\sqrt{2 \pi} \sigma_{v}} \exp \left\{\frac{-v_{i}^{2}}{2 \sigma_{v}^{2}}\right\} \\
& f\left(u_{i j}\right)=\frac{1}{\sqrt{2 \pi} \sigma_{u}} \exp \left\{\frac{-u_{i j}^{2}}{2 \sigma_{u}^{2}}\right\} \\
& f\left(y_{i j} \mid v_{i}, u_{i j}\right)=\frac{1}{\sqrt{2 \pi} \sigma_{i j}} \exp \left\{\frac{-\left[y_{i j}-h^{-1}\left(\mathbf{x}_{i j}^{T} \boldsymbol{\beta}+v_{i}+u_{i j}\right)\right]^{2}}{2 \sigma_{i j}^{2}}\right\}
\end{aligned}
$$

In this case, $E\left(\theta_{i j} \mid \mathbf{y}\right)$ can be computed as follows. By (4.12), we have $f\left(v_{i}, \mathbf{u}_{i} \mid \mathbf{y}_{i}\right)=f\left(v_{i}\right)\left\{\prod_{j=1}^{n_{i}} f\left(y_{i j} \mid u_{i j}, v_{i}\right)\right\}\left\{\prod_{j=1}^{n_{i}} f\left(u_{i j}\right)\right\}$. 
Therefore,

$$
\begin{gathered}
f\left(\mathbf{y}_{i}, v_{i}, \mathbf{u}_{i}\right)=\frac{1}{(2 \pi)^{n_{i}+1 / 2} \sigma_{v} \sigma_{u}^{n_{i}} \prod_{j=1}^{n_{i}} \sigma_{i j}} \times \exp \left\{\frac{-v_{i}^{2}}{2 \sigma_{v}^{2}}-\frac{-\sum_{j=1}^{n_{i}} u_{i j}^{2}}{2 \sigma_{u}^{2}}\right\} \times \\
\exp \left\{\frac{-1}{2} \sum_{j=1}^{n_{i}} \frac{\left[y_{i j}-h^{-1}\left(\mathbf{x}_{i j}^{T} \boldsymbol{\beta}+v_{i}+u_{i j}\right)\right]^{2}}{\sigma_{i j}^{2}}\right\} .
\end{gathered}
$$

By (4.13), we have:

$$
\begin{aligned}
\tilde{\theta}_{i j}(\boldsymbol{\eta}) & =\int \theta_{i j} f\left(v_{i}, \mathbf{u}_{i} \mid \mathbf{y}\right) d v_{i} d \mathbf{u}_{i} \\
& =\frac{\int \theta_{i j} f\left(\mathbf{y}_{i}, v_{i}, \mathbf{u}_{i}\right) d v_{i} d \mathbf{u}_{i}}{f\left(\mathbf{y}_{i}\right)} \\
& =\frac{\int \theta_{i j} f\left(\mathbf{y}_{i}, v_{i}, \mathbf{u}_{i}\right) d v_{i} d \mathbf{u}_{i}}{\int f\left(\mathbf{y}_{i}, v_{i}, \mathbf{u}_{i}\right) d v_{i} d \mathbf{u}_{i}}
\end{aligned}
$$

The above numerator and denominator can be computed separately using the Monte-Carlo integration, as follows.

Let $w^{\star}=\left(v_{i}^{\star}, u_{i 1}^{\star}, u_{i 2}^{\star}, \ldots, u_{i n_{i}}^{\star}\right)$ be a random vector with $w^{\star} \sim N\left(0, \operatorname{diag}\left(\sigma_{v}^{2}, \sigma_{u}^{2} \mathbf{1}_{n i}^{T}\right)\right)$. Using normality assumption, $g\left(w^{\star}\right)$ can be expressed as follows:

$$
g\left(w^{\star}\right)=(2 \pi)^{-n_{i} / 2}\left(\prod_{j=1}^{n_{i}} \sigma_{i j}\right)^{-1} \times \exp \left\{-\sum_{j=1}^{n_{i}} \frac{1}{\sigma_{i j}^{2}}\left[y_{i j}-h^{-1}\left(\mathbf{x}_{i j}^{T} \boldsymbol{\beta}+v_{i}^{\star}+u_{i j}^{\star}\right)\right]^{2}\right\} .
$$

Hence, the denominator can be calculated by $\int g\left(w^{\star}\right) f\left(w^{\star}\right) d w^{\star}$. Similarly, the numerator of (4.17) can be expressed as $\int h^{-1}\left(x_{i j} \beta+v_{i}^{\star}+u_{i j}^{\star}\right) g\left(w^{\star}\right) f\left(w^{\star}\right) d w^{\star}$.

In other words, for computing $\tilde{\theta}_{i j}(\boldsymbol{\eta})$, we only need to generate random variable $w^{\star}$ from $N\left(0, \operatorname{diag}\left(\sigma_{v}^{2}, \sigma_{u}^{2} \mathbf{1}_{n i}^{T}\right)\right)$. 


\subsubsection{Special case II: matched two-fold model with normal random effects}

Here, we consider the case where $h(t) \equiv t$ and the sampling model and the linking are matched. Under normality assumption, $\left(\mathbf{y}_{i}, v_{i}, \mathbf{u}_{i}\right)$ has a multivariate normal distribution, and the analytical form of the density $f\left(\mathbf{y}_{i}, v_{i}, \mathbf{u}_{i}\right)$ can be derived easily.

$$
\begin{aligned}
E\left(\theta_{i j} \mid \mathbf{y}\right) & =E\left(\mathbf{x}_{i j}^{T} \boldsymbol{\beta}+v_{i}+u_{i j} \mid \mathbf{y}_{i}\right) \\
& =\mathbf{x}_{i j}^{T} \boldsymbol{\beta}+E\left(v_{i} \mid \mathbf{y}_{i}\right)+E\left(u_{i j} \mid \mathbf{y}_{i}\right)
\end{aligned}
$$

Let $\boldsymbol{\epsilon}_{i}=\left(\epsilon_{i 1}, \ldots, \epsilon_{i n_{i}}\right)^{T}$, then $\left(v_{i}, \mathbf{u}_{i}^{T}, \epsilon_{i}^{T}\right)^{T} \sim N\left(0, \operatorname{diag}\left(\sigma_{v}^{2}, \sigma_{u}^{2} \mathbf{1} n_{i}, \sigma_{i 1}^{2}, \ldots, \sigma_{i n_{i}}^{2}\right)\right.$. Vector $\left(\mathbf{y}_{i}^{T}, v_{i}, \mathbf{u}_{i}^{T}\right)^{T}$ is a linear combination of $\left(v_{i}, \mathbf{u}_{i}^{T}, \epsilon_{i}^{T}\right)^{T}$ :

$$
\left[\begin{array}{l}
\mathbf{y}_{i} \\
v_{i} \\
\mathbf{u}_{i}
\end{array}\right]=\mathbf{A}_{\left(2 n_{i}+1\right) \times\left(2 n_{i}+1\right)}\left[\begin{array}{l}
v_{i} \\
\mathbf{u}_{i} \\
\boldsymbol{\epsilon}_{i}
\end{array}\right]+\mathbf{B},
$$

where

$$
\mathbf{A}=\left[\begin{array}{ccc}
\mathbf{1}_{n_{i} \times 1} & \mathbf{I}_{n_{i}} & I \mathbf{I}_{n_{i}} \\
1 & \mathbf{0}_{n_{i} \times n_{i}} & \mathbf{0}_{n_{i} \times n_{i}} \\
\mathbf{0}_{n_{i} \times 1} & \mathbf{I}_{n_{i}} & 0_{1 \times n_{i}}
\end{array}\right]
$$

and $\mathbf{B}=\left(\mathbf{x}_{i 1}^{T} \boldsymbol{\beta}, \ldots, \mathbf{x}_{i n_{i}}^{T} \boldsymbol{\beta}, \mathbf{0}_{n_{i}+1 \times 1}\right)^{T}$. 
By multivariate normal theory, we have:

$$
\left[\begin{array}{l}
\mathbf{y}_{i} \\
v_{i} \\
\mathbf{u}_{i}
\end{array}\right] \sim N\left(\mathbf{B}, \mathbf{A} \boldsymbol{\Sigma} \mathbf{A}^{T}\right)
$$

where $\boldsymbol{\Sigma}=\operatorname{diag}\left(\sigma_{v}^{2}, \sigma_{u}^{2} \mathbf{1} n_{i}, \sigma_{i 1}^{2}, \ldots, \sigma_{i n_{i}}^{2}\right)$ is the covariance matrix of $\left(v_{i}, \mathbf{u}_{i}^{T}, \epsilon_{i}^{T}\right)^{T}$.

Given the normality of $\left(\mathbf{y}_{i}, v_{i}, \mathbf{u}_{i}\right)$, one can easily obtain $E\left(v_{i} \mid \mathbf{y}_{i}\right)$. Consequently, $E\left(\theta_{i j} \mid \mathbf{y}\right)$ can be found by

$$
E\left(\theta_{i j} \mid \mathbf{y}\right)=\mathbf{x}_{i j}^{T} \boldsymbol{\beta}+E\left(v_{i} \mid \mathbf{y}_{i}\right)+E\left(u_{i j} \mid \mathbf{y}_{i}\right)
$$

The results above agree with the ones given by Torabi and Rao (2014):

1. The variance matrix of $\mathbf{y}_{i}$, which is obtained as the sub-matrix of $\mathbf{A} \boldsymbol{\Sigma} \mathbf{A}^{T}$ in (4.18) is exactly the same as $\mathbf{V}_{i}$ in Torabi and Rao (2014) as shown in (4.4).

2. The $E\left(v_{i} \mid \mathbf{y}_{i}\right)$ and $E\left(u_{i j} \mid \mathbf{y}_{i}\right)$ are the same as $\tilde{v}_{i}(\delta)$ and $\tilde{u}_{i j}(\delta)$ in Torabi and Rao, 2014 (see (4.6) and (4.5)).

3. The EB estimator given in (4.19) is exactly the same as $\tilde{\theta}_{i j}$ in Torabi and Rao, 2014, as shown in (4.7).

Indeed, the subarea model developed by Torabi and Rao (2014) can be seen as a special case of our general theory defined for any monotonic function $h(\cdot)$. 


\subsection{Estimation of mean squared error}

Estimation of mean squared error (MSE) of small area estimators is a complicated process even for ordinary EBLUP estimators. For the case of EBLUP under a model with block diagonal covariance structure, Prasad and Rao (1990) proposed a linearization method, Jiang, Lahiri, and Wan (2002) studied a jackknife method, and Hall and Maiti (2006) developed a bootstrap method.

Given the complex form of EB estimators discussed in the previous section, it is not possible to directly adopt the existing methods. We propose a parametric bootstrap method, based on estimates $\hat{\boldsymbol{\eta}}=\left(\hat{\boldsymbol{\beta}}, \hat{\boldsymbol{\eta}}_{v}, \hat{\boldsymbol{\eta}}_{u}\right)$ to estimate the MSE of the EBLUP $\hat{\theta}_{i j}$,

$$
\operatorname{MSE}\left(\hat{\theta}_{i j}\right)=E\left\{\left(\hat{\theta}_{i j}-\theta_{i j}\right)^{2}\right\}
$$

In short, the parametric bootstrap method consists of generating parametrically large number of bootstrap samples from the model fitted to the original data, re-estimating the model parameters for each bootstrap sample and then approximating the MSE by MC integration using the bootstrap replicates. This is a useful method, in particular for MSE of complex estimators. For basic area level models, the bootstrap MSE estimation and some of its extensions are discussed in Section 6.2.4 of Rao and Molina (2015).

The proposed parametric bootstrap method consists of the following step:

1. Given the MLE $\hat{\boldsymbol{\eta}}=\left(\hat{\boldsymbol{\beta}}, \hat{\boldsymbol{\eta}}_{v}, \hat{\boldsymbol{\eta}}_{u}\right)$, generate area specific random effect, $v_{i}^{\star}$, subarea specific random effect, $u_{i j}^{\star}$, and random errors, $e_{i j}^{\star}$, from the corresponding distribution:

$v_{i}^{\star} \stackrel{i i d}{\sim} f\left(v ; \hat{\boldsymbol{\eta}}_{v}\right), u_{i j}^{\star} \stackrel{i i d}{\sim} f\left(u ; \hat{\boldsymbol{\eta}}_{u}\right)$ and $e_{i j}^{\star} \stackrel{i i d}{\sim} N\left(0, \psi_{i j}\right)$. 
2. Obtain the corresponding bootstrap subarea parameter,

$$
\theta_{i j}^{\star}=h^{-1}\left(\mathbf{x}_{i j}^{T} \hat{\boldsymbol{\beta}}+v_{i}^{\star}+u_{i j}^{\star}\right)+\epsilon_{i j}^{\star}, \quad i=1, \cdots, m, \quad j=1, \cdots, n_{i} .
$$

3. Create bootstrap observations, $y_{i j}^{\star}$ from the following model

$$
y_{i j}^{\star}=\theta_{i j}^{\star}+e_{i j}^{\star}, \quad i=1, \cdots, m, \quad n_{i} .
$$

4. Repeat steps 1-3 B times to obtain B copies of bootstrap data $\left\{y_{i j}^{\star}{ }^{(b)}\right\}, b=$ $1, \cdots, B$.

5. Based on $\left\{y_{i j}^{\star}{ }^{(b)}\right\}$, compute bootstrap EBLUP estimates $\left\{{\hat{\theta_{i j}^{\star}}}^{(b)}\right\}$ for $b=$ $1, \cdots, B$

6. Obtain a bootstrap estimate of MSE as follows

$$
\operatorname{MSE} E^{B}\left(\hat{\theta}_{i j}\right)=\frac{1}{B} \sum_{b=1}^{B}\left({\hat{\theta_{i j}^{\star}}}^{(b)}-\theta_{i j}^{\star(b)}\right)^{2}
$$

The main advantage of the parametric bootstrap method is its simplicity to implement. However, it should be noted that it may produce biased inference if the underlying distributions are misspecified.

\subsection{Unmatched three-fold model}

In this section, we consider a three-fold model under unmatched linking and sampling models. Under a three fold model, the target population is divided into $M$ areas and each area $i$ is further divided into $N_{i}$ subareas. Subsequently, each sub- 
area $j$ is divided into $L_{i j}$ sub-subareas. The proposed method for an unmatched three-fold model is a natural extension of the the method described in the previous chapter.

The goal is to estimate area mean, $\theta_{i}$, subarea mean, $\theta_{i j}$, as well as the subsubarea mean, $\theta_{i j k}$, by borrowing strength from related areas, subareas and subsubareas. For example, the three level of estimation could be defined based on census geography:

1. Province (i.e., area),

2. Census division (i.e., subarea),

3. Census subdivision (i.e., sub-subarea),

where census division (CD), as defined by Statistics Canada, is a general term for a group of neighbouring municipalities joined together for the purposes of regional planning and managing common services. Census subdivision (CSD) is the general term for municipalities or areas treated as municipal equivalents for statistical purposes (e.g., Indian reserves, Indian settlements and unorganized territories) . For instance, there are 18 CD's and 96 CSD's in Nova Scotia.

We assume that a sample of $m$ areas are taken from $M$ areas. In the second stage of sampling, $n_{i}$ subareas are selected from $N_{i}$ subareas in area $i$. Then, a sample of $l_{i j}$ sub-subareas from subarea $j$ in area $i$ is taken. The sampling model is

$$
y_{i j k}=\theta_{i j k}+e_{i j k}
$$

where $y_{i j k}$ is the direct estimator of $\theta_{i j k}$ with sampling error $e_{i j k}$, and $\epsilon_{i j k} \stackrel{i n d}{\sim}$ $N\left(0, \psi_{i j k}\right)$. We assume that $\psi_{i j k}$ 's are known. 
The linking model for the sub-subarea means $\theta_{i j k}$ is

$h\left(\theta_{i j k}\right)=\mathbf{x}_{i j k}^{T} \boldsymbol{\beta}+v_{i}+u_{i j}+w_{i j k}, \quad i=1, \cdots, m ; \quad j=1, \cdots, n_{i}, \quad k=1, \cdots, l_{i j}$.

where $h(\cdot)$ is a monotonic link function, $\mathbf{x}_{i j k}$ is the covariate vector associated with $y_{i j k}$ and known for all sub-subareas, $v_{i} \stackrel{i i d}{\sim} f\left(v ; \boldsymbol{\eta}_{v}\right)$ represents the area random effect, $u_{i j} \stackrel{\text { iid }}{\sim} f\left(u ; \boldsymbol{\eta}_{u}\right)$ is the subarea random effect, and $w_{i i j} \stackrel{i i d}{\sim} f\left(w ; \boldsymbol{\eta}_{w}\right)$ denotes the sub-subarea random effect. We assume that $v_{i}, u_{i j}$ and $w_{i j k}$ are mutually independent.

Similar to the unmatched two-fold case, we will obtain the empirical best (EB) of $\theta_{i j k}$. We use the following notation:

- $\mathbf{y}_{i}=\left(\mathbf{y}_{i 1}, \cdots, \mathbf{y}_{i n_{i}}\right)^{T}$ is $n_{i} \times 1$ vector of direct estimators, where $\mathbf{y}_{i j}=$ $\left(y_{i j 1}, \cdots, y_{i j l_{i j}}\right)^{T}, i=1, \cdots, m, j=1, \cdots, n_{i}$,

- $\mathbf{u}_{i}=\left(u_{i 1}, \cdots, u_{i n_{i}}\right)^{T}, i=1, \cdots, m$ is the subarea-level random effects

- $\mathbf{w}_{i}=\left(\mathbf{w}_{i 1}, \cdots, \mathbf{w}_{i n_{i}}\right)^{T}$ is a $n_{i} \times 1$ vector of sub-subarea-level random effects, where $\mathbf{w}_{i j}=\left(w_{i j 1}, \cdots, w_{i j l_{i j}}\right), i=1, \cdots, m, j=1, \cdots, n_{i}$

- $\boldsymbol{\eta}=\left(\boldsymbol{\beta}, \boldsymbol{\eta}_{\boldsymbol{v}}, \boldsymbol{\eta}_{u}, \boldsymbol{\eta}_{w}\right)$ is the model parameters.

For simplicity, we assume that all areas are sampled, $M=m$. Depending on values of $j$ and $k$, there will be three possible cases:

- Case I: sampled sub-subarea within a sampled subarea

- Case II: non-sampled sub-subarea within a sampled subarea

- Case III: non-sampled sub-subarea within a non-sampled subarea 
From (4.21), $\theta_{i j k}$ is a function of $v_{i}, u_{i j}$ and $w_{i j k}$. the $\mathrm{BP}$ of $\theta_{i j k}$ is given by conditional expectation of $\theta_{i j k}$ given $\mathbf{y}=\left\{y_{i j k}, i=1, \cdots, m, \quad j=1, \cdots, n_{i}, \quad k=\right.$ $\left.1, \cdots, l_{i j}\right\}$.

$$
\tilde{\theta}_{i j k}=E\left(\theta_{i j k} \mid \mathbf{y} ; \boldsymbol{\eta}\right)
$$

\section{Case I: sampled sub-subarea within a sampled subarea}

In the case of sampled subareas and sampled sub-subareas $j=1, \cdots, n_{i}$ and $k=1, \cdots, l_{i j}$, we obtain $f\left(\mathbf{y}_{i}\right)$ by integrating with respect to $v_{i}, \mathbf{u}_{i}$ and $\mathbf{w}_{i}$.

$$
\theta_{i j k}=h^{-1}\left(\mathbf{x}_{i j k}^{T} \boldsymbol{\beta}+v_{i}+u_{i j}+w_{i j k}\right)
$$

Therefore, the best predictor $(\mathrm{BP})$ of $\theta_{i j k}$ can be written as:

$$
\begin{aligned}
\tilde{\theta}_{i j k}(\boldsymbol{\eta}) & =E\left(\theta_{i j k} \mid \mathbf{y}\right) \\
& =\int \theta_{i j k} f\left(v_{i}, \mathbf{u}_{i}, \mathbf{w}_{i} \mid \mathbf{y}_{i} ; \boldsymbol{\eta}\right) d v_{i} d \mathbf{u}_{i} d \mathbf{w}_{i} \\
& =\int \theta_{i j k} \frac{f\left(v_{i}, \mathbf{u}_{i}, \mathbf{w}_{i}, \mathbf{y}_{i} ; \boldsymbol{\eta}\right)}{f\left(\mathbf{y}_{i}\right)} d v_{i} d \mathbf{u}_{i} d \mathbf{w}_{i} \\
& =\frac{\int \theta_{i j k} f\left(v_{i}, \mathbf{u}_{i}, \mathbf{w}_{i}, \mathbf{y}_{i} ; \boldsymbol{\eta}\right) d v_{i} d \mathbf{u}_{i} d \mathbf{w}_{i}}{\int\left(v_{i}, \mathbf{u}_{i}, \mathbf{w}_{i}, \mathbf{y}_{i} ; \boldsymbol{\eta}\right) d v_{i} d \mathbf{u}_{i} d \mathbf{w}_{i}}
\end{aligned}
$$

We need to compute $G(\boldsymbol{\eta})$ :

$$
\begin{aligned}
G(\boldsymbol{\eta}) & :=\int g\left(v_{i}, \mathbf{u}_{i}, \mathbf{w}_{i}\right) f\left(v_{i}, \mathbf{u}_{i}, \mathbf{w}_{i}, \mathbf{y}_{i} ; \boldsymbol{\eta}\right) d v_{i} d \mathbf{u}_{i} d \mathbf{w}_{i} \\
& =\int g\left(v_{i}, \mathbf{u}_{i}, \mathbf{w}_{i}\right) \frac{f\left(v_{i}, \mathbf{u}_{i}, \mathbf{w}_{i}, \mathbf{y}_{i} ; \boldsymbol{\eta}\right)}{f\left(v_{i}, \mathbf{u}_{i}, \mathbf{w}_{i} ; \boldsymbol{\eta}\right)} f\left(v_{i}, \mathbf{u}_{i}, \mathbf{w}_{i} ; \boldsymbol{\eta}\right) d v_{i} d \mathbf{u}_{i} d \mathbf{w}_{i} \\
& =\int\left\{g\left(v_{i}, \mathbf{u}_{i}, \mathbf{w}_{i}\right) f\left(\mathbf{y}_{i} \mid v_{i}, \mathbf{u}_{i}, \mathbf{w}_{i} ; \boldsymbol{\eta}\right)\right\} f\left(v_{i}, \mathbf{u}_{i}, \mathbf{w}_{i} ; \boldsymbol{\eta}\right) d v_{i} d \mathbf{u}_{i} d \mathbf{w}_{i}
\end{aligned}
$$


$G(\boldsymbol{\eta})$ can be computed using MC integration by generating $\left(v_{i, t}^{\star}, \mathbf{u}_{i, t}^{\star}, \mathbf{w}_{i, t}^{\star}\right), \quad t=$ $1, \cdots, T$ from $f\left(v_{i}, \mathbf{u}_{i}, \mathbf{w}_{i}\right)$, where $\mathrm{T}$ is a large number. In practice, assuming that random effects $\left(v_{i}, \mathbf{u}_{i}\right.$ and $\left.\mathbf{w}_{i}\right)$ are coming from normal distribution, $f\left(v_{i}, \mathbf{u}_{i}, \mathbf{w}_{i}\right)$ can be easily generated as $v_{i}, \mathbf{u}_{i}$ and $\mathbf{w}_{i}$ are mutually independent. Moreover, if we fix $v_{i}, \mathbf{u}_{i}$ and $\mathbf{w}_{i}, \theta_{i j k}$ will be fixed and its distribution will be normal.

$$
G(\boldsymbol{\eta}) \approx \frac{1}{T} g\left(v_{i, t}^{\star}, \mathbf{u}_{i, t}^{\star}, \mathbf{w}_{i, t}^{\star}\right) f\left(\mathbf{y}_{i} \mid v_{i, t}^{\star}, \mathbf{u}_{i, t}^{\star}, \mathbf{w}_{i, t}^{\star} ; \boldsymbol{\eta}\right)
$$

Once we obtain the BP of $\theta_{i j k}$, we will find the MLE $\hat{\boldsymbol{\eta}}$ of the parameters $\boldsymbol{\eta}$. Then EB of $\theta_{i j k}$ is given by $\hat{\theta}_{i j k}=\tilde{\theta}_{i j k}(\hat{\boldsymbol{\eta}})$.

\section{Case II: non-sampled sub-subarea within a sampled subarea}

In the case of sampled subareas and non-sampled sub-subareas, where $j=$ $1, \cdots, n_{i}$ and $l=l_{i j}+1, \cdots, L_{i j}$, and $l \neq k, w_{i j l}$ is independent of $\left(\mathbf{y}_{i}, v_{i}, \mathbf{u}_{i}, \mathbf{w}_{i}\right)$.

Hence, $\tilde{\theta}_{i j l}(\boldsymbol{\eta})$ can be calculated as follows

$$
\begin{aligned}
\tilde{\theta}_{i j l}(\boldsymbol{\eta}) & =\int \theta_{i j l} f\left(v_{i}, u_{i j}, w_{i j l} \mid \mathbf{y}_{i} ; \boldsymbol{\eta}\right) d v_{i} d u_{i j} d w_{i j l} \\
& =\int \theta i j l f\left(v_{i}, \mathbf{u}_{i}, \mathbf{w}_{i}, w_{i j l} \mid \mathbf{y}_{i} ; \boldsymbol{\eta}\right) d v_{i} d \mathbf{u}_{i} d u_{i j} d w_{i j l} \\
& =\frac{\int \theta_{i j l} f\left(v_{i}, \mathbf{u}_{i}, \mathbf{w}_{i}, \mathbf{y}_{i} ; \boldsymbol{\eta}\right) f\left(w_{i j l} ; \boldsymbol{\eta}\right) d v_{i} d \mathbf{u}_{i} d w_{i j l}}{\int f\left(\mathbf{y}_{i}, v_{i}, \mathbf{u}_{i}, \mathbf{w}_{i} ; \boldsymbol{\eta}\right) d v_{i} d \mathbf{u}_{i} \mathbf{w}_{i}}
\end{aligned}
$$

The above integration can be computed through MC integration as discussed in the previous section. 


\section{Case III: non-sampled sub-subarea within a non-sampled subarea}

For a non-sampled subarea, " $o$ " where $o=\left(n_{i}+1\right), \cdots, N_{i}$, and $l=1, \cdots, L_{i o}$, $\left(u_{i o}, w_{i o l}\right)$ is independent of $\mathbf{y}$.

$$
f\left(u_{i o}, w_{i o l} \mid \mathbf{y}\right)=f\left(u_{i o}, w_{i o l}\right)=f\left(u_{i o}\right) f\left(w_{i o l}\right)
$$

$E\left(\theta_{k l} \mid \mathbf{y}\right)$ can be obtained as follows:

$$
E\left(\theta_{i o l} \mid \mathbf{y}\right)=\int \theta_{i o l} f\left(u_{i o}\right) f\left(w_{i o l}\right) d u_{i o} d_{w i o l}
$$

For a given $\boldsymbol{\eta}$, data $\mathbf{y}$ does not play a role in the estimation of $\theta_{i o l}$. Similar to previous cases, MC integration can be used for computing the $E\left(\theta_{\text {iol }} \mid \mathbf{y}\right)$.

After obtaining the BP, we should estimate the model parameters $\boldsymbol{\eta}=$ $\left(\boldsymbol{\beta}, \boldsymbol{\eta}_{\boldsymbol{v}}, \boldsymbol{\eta}_{u}, \boldsymbol{\eta}_{w}\right)$. Similar to the two-fold model, we use MLE:

$$
\hat{\boldsymbol{\eta}}=\underset{\eta}{\arg \max } L(\boldsymbol{\eta})
$$

where $L(\boldsymbol{\eta})=\prod_{i=1}^{m} f\left(\mathbf{y}_{i} ; \boldsymbol{\eta}\right)$, with

$$
f\left(\mathbf{y}_{i} ; \boldsymbol{\eta}\right)=\int f\left(v_{i}, \mathbf{u}_{i}, \mathbf{w}_{i}, \mathbf{y}_{i} ; \boldsymbol{\eta}\right) d v_{i} d \mathbf{u}_{i} d \mathbf{w}_{i} \approx \frac{1}{T} \sum_{i=1}^{m} f\left(\mathbf{y}_{i} \mid v_{i, t}^{\star}, \mathbf{u}_{i, t}^{\star}, \mathbf{w}_{i, t}^{\star} ; \boldsymbol{\eta}\right),
$$

where $\left(v_{i, t}^{\star}, \mathbf{u}_{i, t}^{\star}, \mathbf{w}_{i, t}^{\star}\right)$ are generated from $f\left(v_{i}, \mathbf{u}_{i}, \mathbf{w}_{i} ; \boldsymbol{\eta}\right)$.

Finally, the EB estimator of sub-subarea parameter is obtained by plugging $\hat{\boldsymbol{\eta}}$ into the expression of the BP. 


\subsection{Simulation Study}

We now conduct a simulation study to evaluate the performance of the EB estimators of means of sampled and non-sample subareas under unmatched two-fold models.

We set the total number of areas to 50 . Areas number 1-15 (area group 1) each contain 20 subareas, areas number 16-35 (area group 2) each contain 30 subareas, and areas number 36-50 (area group 3) each contain 15 subareas. The number of sampled areas is set to 30 , with 10 of them from area group 1 each having 8 sampled subareas, 15 of them from area group 2 each having 5 sampled subareas, and 5 of them from area group 3 each having 10 sampled subareas. The number of simulation repetitions is set to 5000 . The number of random draws in each MC integration is set to 30000 to ensure approximation accuracy.

We consider two different settings for the link function: logit and log. Under each setting of the link function, we compare the performance of the unmatched two-fold model to that of the unmatched one-fold model (Sugasawa et al. (2018)) at subarea level by ignoring the area-level random effect $v_{i}$ in the linking model (4.9).

For the case of the logit link function, subarea data $y_{i j}$ are generated under the sampling model (4.8) and linking model

$$
h\left(\theta_{i j}\right)=\beta_{0}+\beta_{1} x_{i j}+v_{i}+u_{i j},
$$

where $h(\cdot)=\operatorname{logit}(\cdot), \beta_{0}=0.1, \beta_{1}=0.2, v_{i} \sim N\left(0, \sigma_{v}^{2}\right)$ with $\sigma_{v}=0.8, u_{i j} \sim$ $N\left(0, \sigma_{u}^{2}\right)$ with $\sigma_{u}=0.4$, and $x_{i j}$ is a single covariate generated from $\operatorname{Gamma}(3,0.5)$, 
a gamma distribution with shape parameter 3 and scale parameter 0.5 . The sampling variances $\psi_{i j}$ are generated from $0.04+\operatorname{Gamma}(0.5,0.1)$.

For the case of a log link function, subarea data $y_{i j}$ are generated with sampling model (4.8) and the linking model (4.25) with $h(\cdot)=\log (\cdot), \beta_{0}=-1, \beta_{1}=$ $0.5, v_{i} \sim N\left(0,0.8^{2}\right), u_{i j} \sim N\left(0,0.4^{2}\right)$, and $x_{i j} \sim \operatorname{Gamma}(1,0.5)$. The sampling variances $\psi_{i j}$ are generated from $0.1+\operatorname{Gamma}(0.5,0.2)$.

We report simulation results for the EB estimation of three different types of subareas separately: sampled subareas within sampled areas (S-S), non-sampled subareas within sampled areas (N-S), and non-sampled subareas within nonsampled areas $(\mathrm{N}-\mathrm{N})$. For each type of subareas, we report the average absolute bias (AABIAS) given by

$$
\text { AABIAS }=\frac{1}{n} \sum_{i} \sum_{j}\left|\frac{1}{T} \sum_{t=1}^{T}\left(\hat{\theta}_{i j}^{(t)}-\theta_{i j}^{(t)}\right)\right|,
$$

and the average root mean-squared error (ARMSE) given by

$$
\operatorname{ARMSE}=\frac{1}{n} \sum_{i} \sum_{j}\left\{\frac{1}{T} \sum_{t=1}^{T}\left(\hat{\theta}_{i j}^{(t)}-\theta_{i j}^{(t)}\right)^{2}\right\}^{1 / 2},
$$

where $n$ is the total number of subareas under consideration, $T$ is the total number of simulation replications, and $\theta_{i j}^{(t)}$ and $\hat{\theta}_{i j}^{(t)}$ are the true and estimated subarea mean in $t^{\text {th }}, t=1, \ldots, T$, simulation replication, respectively.

The simulation results for EB estimation of subarea means using unmatched two-fold model and unmatched one-fold model are reported in Table 4.1. 
Table 4.1: EB for subarea means under unmatched two fold-models

\begin{tabular}{||l|c|cc|cc|ccc||}
\hline \multirow{2}{*}{ Link } & \multirow{2}{*}{ Model } & \multicolumn{2}{|c|}{ S-S } & \multicolumn{2}{|c||}{ N-S } & \multicolumn{2}{||}{ N-N } \\
& & AABIAS & ARMSE & AABIAS & ARMSE & AABIAS & ARMSE \\
\hline \hline \multirow{2}{*}{$\operatorname{logit}$} & two-fold & 0.00184 & 0.0506 & 0.00170 & 0.0958 & 0.00185 & 0.188 \\
& one-fold & 0.00236 & 0.0552 & 0.00245 & 0.185 & 0.00256 & 0.189 \\
\hline $\log$ & two-fold & 0.0339 & 0.222 & 0.0140 & 0.588 & 0.0811 & 0.903 \\
& one-fold & 0.0319 & 0.210 & 0.0200 & 0.932 & 0.0786 & 0.894 \\
\hline
\end{tabular}

In the S-S and N-N cases, the one-fold and two-fold models perform equally well under both logit and log link functions. This is likely because both models use the sampled data $y_{i j}$ in the $\mathrm{S}-\mathrm{S}$ case, and neither model uses $y_{i j}$ in the N-N case. However, in the N-S case, the two-fold model clearly outperforms the onefold model, which is due to the fact that only the two-fold model uses the sampled data through the area-level random effect $v_{i}$ in this case.

\subsection{Summary}

In this chapter, we studied two-fold subarea level model under unmatched linking and sampling models. We proposed an EB approach to estimation of small subarea means. We described the method in three main steps: 1) deriving the BP of small subarea means, 2) obtaining the MLE of the model parameters and 3) computing the EB of small subarea means by substituting the model parameters in the BP 
estimator found in the first step. We also studied estimation of non-sampled subareas within non-sampled areas, and proposed a parametric bootstrap method to estimate the MSE of $\hat{\theta}_{i j}$. The proposed method was extended to a three-fold model under unmatched linking and sampling models. A Monte Carlo simulation was conducted to evaluate the performance of EB estimators of means sampled and non-sampled areas under unmatched two-fold and one-fold model. The simulation results showed that, for non-sampled subareas within sampled areas, the two-fold model performs better than the one-fold model. 


\section{Chapter 5}

\section{Variable Selection under two-fold subarea model}

The main objective of variable selection methods is to find the best set of covariates to be used in the model so as to increase prediction and estimation accuracy. The primary assumption behind standard variable selection methods is that the variable of interest is observed, which is not the case for models used in small area estimation, as dependent variables (e.g., area means) are not observed due to the sampling errors. In particular, for a two-fold subarea level model, where the parameter of interest is subarea means $\left(\theta_{i j}\right)$, direct estimates of subarea means $\left(y_{i j}\right)$ are measured as proxies for unknown $\theta_{i j}$. In this situation, applying standard regression model selection may result in an inefficient model.

In this chapter, we propose a two-step adjustment to the standard variable selection method for two-fold subarea level models. Section 5.1 gives a brief introduction to variable selection methods in the context of regression analysis and reviews some of the commonly used variable selection criteria. We discuss some 
recent work in this area in Section 5.2. The proposed method is introduced in Section 5.3, and results of a simulation study are reported in Section 5.4. Concluding remarks and some proofs are given in Section 5.5 and Section 5.6.

\section{$5.1 \quad$ Introduction}

Variable selection for mixed models has been extensively studied in the literature (Lin (1997), Vaida and Blanchard (2005), Liang et al. (2008)). In particular, Ibrahim et al. (2011) developed simultaneous fixed and random effects selection procedure based on the smoothly clipped absolute deviation (SCAD) and adaptive least absolute shrinkage and selection operator (ALASSO) using maximum penalized likelihood (MPL) estimation. Most of the proposed methods consider selecting both fixed and random effects in a general class of mixed effects models with applications in longitudinal models and correlated models. However, in small area estimation, where random effects are not usually subject to selection, users often apply variable selection methods that ignore random effects. As mentioned earlier, naively applying standard regression model selection may result in an inefficient model.

In general, a regression model relates variable of interest, $\mathbf{y}=\left(y_{1}, y_{2}, \cdots, y_{n}\right)^{T}$, to a function of covariates, $\mathbf{X}=\left(\mathbf{x}_{1}, \cdots, \mathbf{x}_{p}\right)$, where $\mathbf{x}_{i}=\left(x_{i 1}, x_{i 2}, \cdots, x_{i n}\right)^{T}$. For linear regression, the model can be expressed as

$$
\mathbf{y}=\mathbf{X} \boldsymbol{\beta}+\boldsymbol{\epsilon}
$$

where $\mathbf{X}$ is a $n \times p$ matrix, $\boldsymbol{\beta}$ is a $p \times 1$ vector of regression coefficients, and $\boldsymbol{\epsilon}$ is an $n \times 1$ vector of errors, with $\boldsymbol{\epsilon} \sim N\left(\mathbf{0}, \sigma^{2} \mathbf{I}\right)$. 
The goal of variable selection is to identify the best subset of variables among $k$ available covariates (or predictors) to be included in the model. Several variable selection methods are available in regression analysis. Some commonly used variable selection criteria are:

- Akaike information criterion (AIC),

- Bayesian information criterion (BIC),

- Adjusted $R^{2}$

- $C_{p}$ statistic.

Using the notation in (5.1), we review $\mathrm{AIC}, \mathrm{BIC}$, adjusted $R^{2}$ and $C_{p}$ in the following sub-sections.

\subsubsection{Akaike information criterion (AIC)}

The Akaike information criterion (AIC) measure was first developed by Akaike (1973) as a way for comparing different models. It is based on the log-likelihood of the model and penalizes free parameters to control over fitting. Under model (5.1), AIC is defined as

$$
A I C=2 p+n \log \left(\frac{S S E}{n}\right)
$$

where

$$
S S E=\mathbf{y}^{T}[\mathbf{I}-\mathbf{P}] \mathbf{y}, \quad \mathbf{P}=\mathbf{X}\left(\mathbf{X}^{T} \mathbf{X}\right)^{-1} \mathbf{X}^{T},
$$


$n$ is the number of observations and $p$ is the number of predictors used in the model. The model with the lowest AIC is the best choice among other tested models.

\subsubsection{Bayesian information criterion (BIC)}

The Bayesian information criterion (BIC) or Schwarz criterion is a criterion for model selection based on log-likelihood of the model. The BIC measure, which was developed by Schwarz (1978), resolves the over-fitting issue by introducing a penalty term for the number of parameters in the model. It is given by

$$
B I C=p \log (n)+n \log \left(\frac{S S E}{n}\right)
$$

where

$$
S S E=\mathbf{y}^{T}[\mathbf{I}-\mathbf{P}] \mathbf{y}, \quad \mathbf{P}=\mathbf{X}\left(\mathbf{X}^{T} \mathbf{X}\right)^{-1} \mathbf{X}^{T},
$$

$n$ is the number of observations and $p$ is the number of predictors used in the model.

The $\mathrm{BIC}$ is an increasing function of SSE and an increasing function of $p$, which means both variation in the data and a large number of auxiliary variables increase the value of BIC. In general, the model with the lower value of BIC is preferred. Compared to the AIC, the BIC penalizes more on a model with more parameters. 


\subsubsection{Adjusted $R^{2}$}

The adjusted R-squared is the revised version of R-squared that accounts for the number of predictors in the model,

$$
R^{2}(a d j)=1-\frac{M S E}{M S T}
$$

where

$$
\begin{aligned}
& M S E=\frac{S S E}{n-p}=\frac{1}{n-p} \mathbf{y}^{T}[\mathbf{I}-\mathbf{P}] \mathbf{y} \\
& M S T=\frac{S S T}{n-1}=\frac{1}{n-1} \mathbf{y}^{T}\left[\mathbf{1}-n^{-1} \mathbf{1 1}^{T}\right] \mathbf{y} \\
& \mathbf{P}=\mathbf{X}\left(\mathbf{X}^{T} \mathbf{X}\right)^{-1} \mathbf{X}^{T}
\end{aligned}
$$

$p$ is the number of predictors used in the model and $n$ is the number of observations. We assume that $\mathbf{X}^{T} \mathbf{X}$ is invertible.

The adjusted R-squared increases only if the new term has a correlation to the variable of interest.

\subsection{4 $C_{p}$ statistic}

$C_{p}$ statistics or Mallows's $C_{p}$ measure was developed by Mallows in 1973 Mallows (1973) to address the over-fitting problem.

$$
C_{p}=\frac{S S E_{p}}{M S E}+2 p-n
$$

where $S S E_{p}$ is the sum of the squares of residuals for a model with $p<k$ covariates and $n$ is the number of observations. In practice, to find an optimum model $C_{p}$ 
statistic is calculated for all possible combinations of variables. The model with the lowest $C_{p}$ measure which is closer to $p$ value is the best choice among other tested models.

\subsection{Variable selection in small area estimation}

A few previous studies investigated the limitation of standard variable selection criteria for some of the models used in small area estimation, and proposed alternative measures. In particular, Meza and Lahiri (2005) studied the $C_{p}$ criterion for nested error regression model and proposed applying a transformation to the data. Lahiri and Suntornchost (2015) proposed an adjustment to the standard variable selection method for the Fay-Herriot model. Li and Lahiri (2018) studied the nested error regression models and suggested a transformation of the data so that the transformed data follow a standard regression model. The next subsection gives a brief review of Lahiri and Suntornchost's paper, which we will be referring to later in this chapter.

\subsubsection{Lahiri and Suntornchost's paper}

Lahiri and Suntornchost (2015) considered the Fay-Herriot model and proposed a new variable selection criterion based on the linking model. They pointed out that standard variable selection methods rely on the assumption that the variable of interest is observed. For the Fay-Herriot model, dependent variable $\theta_{i}$ is not observed, due to the sampling errors $\left(e_{i}\right)$, and is measured instead through $y_{i}=\theta_{i}+e_{i}$. They showed that replacing $\theta_{i}$ with $y_{i}$ and applying standard regression methods may lead to non-optimal or incorrect models. They considered the 
following linking model

$$
\theta_{i}=\mathbf{x}_{i}^{T} \boldsymbol{\beta}+v_{i}, \quad i=1, \ldots, m,
$$

where $\theta_{i}$ is the unobserved true mean for area $i, v_{i}$ 's are the area-specific random effect, which are assumed to be iid normal with mean zero and variance $\sigma_{v}^{2}$. Assuming that direct estimates $y_{i}$ 's are available, the sampling model is expressed as follows:

$$
y_{i}=\theta_{i}+e_{i}, \quad i=1, \cdots, m,
$$

where $e_{i}$ 's are normally-distributed sampling errors with mean zero and known sampling variances $\psi_{i}$.

Lahiri and Suntornchost (2015) proposed a new selection criterion for the linking model (5.4) with unobserved dependent variable. They noted that variable selection measures can be expressed as a function of mean squared error (MSE) and mean of squared total (MST) or $f(M S E, M S T)$, and obtained an approximation using $f\left(M S E_{\theta}, M S T_{\theta}\right)$, where $M S E_{\theta}$ and $M S T_{\theta}$ are MSE and MST when we replace $\mathbf{y}$ with $\boldsymbol{\theta}$.

Let $m$ be the number of areas and $p$ be the number of covariates. Lahiri and Suntornchost defined a new variable selection criterion as

$$
\begin{aligned}
& \widehat{M S E}_{\theta}=M S E-\bar{\psi}_{w}, \\
& \widehat{M S T}_{\theta}=M S T-\bar{\psi},
\end{aligned}
$$

where $\bar{\psi}_{w}=\frac{\sum_{i=1}^{m}\left(1-h_{i i}\right) \psi_{i}}{m-p}$ with $h_{i i}=\mathbf{x}_{i}\left(\mathbf{X}^{T} \mathbf{X}\right)^{-1} \mathbf{x}_{i}$ and $\bar{\psi}=\frac{1}{m} \sum_{i=1}^{m} \psi_{i}$.

Furthermore, they suggested a truncated form of the criterion to avoid negative values that may occur for small $m$. It was shown that the proposed variable 
selection criterion tracks the corresponding ideal criterion, and provides better results compared to the standard variable selection method.

\subsection{Proposed method}

The idea is to make an adjustment to standard variable selection measures so that they can be used with two-fold subarea models in small area estimation. As mentioned in chapter 4, Torabi and Rao (2014) extended Fay and Herriot (1979) area level model to subarea level. The linking model in this case is given by

$$
\theta_{i j}=\mathbf{x}_{i j}^{T} \boldsymbol{\beta}+v_{i}+u_{i j}
$$

where $\mathbf{x}_{i j}$ is a $p \times 1$ vector of subarea level auxiliary variables, $\boldsymbol{\beta}$ is a $p \times 1$ vector of regression parameter, $v_{i} \stackrel{i i d}{\sim} N\left(0, \sigma_{v}^{2}\right)$ is the area effect and $u_{i j}$ is the subarea effect, $u_{i j} \stackrel{i i d}{\sim} N\left(0, \sigma_{u}^{2}\right)$.

It is assumed that $n_{i}$ subareas are sampled from $N_{i}$ subareas in area $i$. The sampling model is given by

$$
y_{i j}=\theta_{i j}+e_{i j}
$$

where $y_{i j}$ is a direct estimator of $\theta_{i j}$ (i.e., $\left.y_{i j}=\hat{\theta}_{i j}\right), e_{i j} \stackrel{i n d}{\sim} N\left(0, \psi_{i j}\right)$, with known $\psi_{i j}$. We assume that $v_{i}, u_{i j}$ and $e_{i j}$ are independent. Combining (5.6) and (5.7), we will get the two-fold subarea level model

$$
y_{i j}=\mathbf{x}_{i j}^{T} \boldsymbol{\beta}+v_{i}+u_{i j}+e_{i j}, \quad i=1, \ldots, m, \quad j=1, \ldots, n_{i} .
$$


We assume that $k$ subarea level auxiliary variables are available. More precisely, for subarea "ij", we have $\mathbf{x}_{i j}=\left(x_{i j 1}, x_{i j 2}, \cdots, x_{i j k}\right)$. The goal is to select the best subset of predictors, say $p$ variables $(p<k)$, among the $k$ available variables.

If $\theta_{i j}$ in (5.6) is known, we can construct any of the criterion discussed in Section 5.1 based on the linking model. However, direct estimates $y_{i j}$ 's are measured instead as proxies for unknown $\theta_{i j}$ 's. So, for constructing the selection measures, we should follow a similar approach as in Lahiri and Suntornchost (2015) and define new variable selection criteria similar to (5.5). However, the two-fold sub-

area model includes two random effects, $v_{i}$ and $u_{i j}$. Hence, we should first apply a transformation adjustment so that the transformed model follows the standard model considered in Lahiri and Suntornchost (2015). As a result, our proposed adjustment to the standard variable selection method for the two-fold model consists of two steps:

1. Transforming the linking model and the sampling model,

2. Obtaining an approximation to the desired regression variable selection criterion for the transformed linking model.

We will explain each of these steps in the next subsections.

\subsubsection{Transformation of the linking and sampling models}

Following Fuller and Battese (1973) and Rao et al. (1993), we apply Fuller-Battese transformation to the linking model. It should be mentioned that Meza and Lahiri (2005) and Li and Lahiri (2018) used the same transformation for nested error regression models. The Fuller-Battese transformation of the linking model (5.6) 
is given by

$$
\theta_{i j}-\alpha_{i} \bar{\theta}_{i}=\left(\mathbf{x}_{i j}-\alpha_{i} \overline{\mathbf{x}}_{i}\right)^{T} \boldsymbol{\beta}+\left(1-\alpha_{i}\right) v_{i}+\left(u_{i j}-\alpha_{i} \bar{u}_{i}\right)
$$

where $\overline{\mathbf{x}}_{i}=\frac{1}{n_{i}} \sum_{j=1}^{n_{i}} \mathbf{x}_{i j}, \bar{u}_{i}=\frac{1}{n_{i}} \sum_{j=1}^{n_{i}} u_{i j}, \bar{\theta}_{i}=\frac{1}{n_{i}} \sum_{j=1}^{n_{i}} \theta_{i j}, \alpha_{i}=1-\left[\frac{1-\rho}{1+\left(n_{i}-1\right) \rho}\right]^{1 / 2}$ with $\rho=\frac{\sigma_{v}^{2}}{\sigma_{v}^{2}+\sigma_{u}^{2}}$. Let $\theta_{i j}^{\star}=\theta_{i j}-\alpha_{i} \bar{\theta}_{i}$ and $\mathbf{x}_{i j}^{\star}=\mathbf{x}_{i j}-\alpha_{i} \overline{\mathbf{x}}_{i}$ and $u_{i j}^{\star}=\left(1-\alpha_{i}\right) v_{i}+$ $\left(u_{i j}-\alpha_{i} \bar{u}_{i}\right)$, then $(5.9)$ can be written as

$$
\theta_{i j}^{\star}=\mathbf{x}_{i j}^{\star T} \boldsymbol{\beta}+u_{i j}^{\star}, \quad i=1, \cdots, m, \quad j=1, \cdots, n_{i} .
$$

Equation (5.10) is transformed linking model. As shown in Fuller and Battese (1973), $u_{i j}^{\star} \stackrel{i i d}{\sim} N\left(0, \sigma_{u}^{2}\right)$, so (5.10) can be seen as an standard regression model.

We transform the sampling model accordingly, so that the linking model and the sampling model can be combined together. The transformation of the sampling model is

$$
y_{i j}-\alpha_{i} \bar{\theta}_{i}=\theta_{i j}-\alpha_{i} \bar{\theta}_{i}+e_{i j}-\alpha_{i} \bar{e}_{i}
$$

where $\bar{e}_{i}=\frac{1}{n_{i}} \sum_{j=1}^{n_{i}} e_{i j}$. Let $y_{i j}^{\star}=y_{i j}-\alpha_{i} \bar{\theta}_{i}$ and $e_{i j}^{\star}=e_{i j}-\alpha_{i} \bar{e}_{i}$, then (5.11) can be written as:

$$
y_{i j}^{\star}=\theta_{i j}^{\star}+e_{i j}^{\star}, \quad i=1, \cdots, m, \quad j=1, \cdots, n_{i} .
$$

The transformed two-fold subarea model can be formed by combining (5.12) and $(5.10)$ :

$$
y_{i j}^{\star}=\mathbf{x}_{i j}^{\star} T \boldsymbol{\beta}+u_{i j}^{\star}+e_{i j}^{\star}, \quad j=1, \cdots, n_{i}, \quad i=1, \cdots, m .
$$


Unlike $e_{i j}$ 's in (5.8), $e_{i j}^{\star}$ 's are not mutually independent. We now need to derive the covariance structure of $e_{i j}^{\star}$.

1. If $j \neq j^{\prime}$ and $i=i^{\prime}$

$$
\begin{aligned}
\operatorname{cov}\left(e_{i j}^{\star}, e_{i^{\prime} j^{\prime}}^{\star}\right) & =\operatorname{cov}\left(e_{i j}^{\star}, e_{i j^{\prime}}^{\star}\right) \\
& =\operatorname{cov}\left(e_{i j}-\alpha_{i} \bar{e}_{i}, e_{i^{\prime} j^{\prime}}-\alpha_{i} \bar{e}_{i}\right) \\
& =\operatorname{cov}\left(e_{i j}, e_{i j^{\prime}}\right)-\alpha_{i} \operatorname{cov}\left(e_{i j} \bar{e}_{i}\right)-\alpha_{i} \operatorname{cov}\left(\bar{e}_{i}, e_{i j^{\prime}}\right)+\alpha_{i}^{2} \operatorname{cov}\left(\bar{e}_{i}, \bar{e}_{i}\right) \\
& =0-\alpha_{i} \frac{1}{n_{i}} \sum_{k=1}^{n_{i}} \operatorname{cov}\left(e_{i j}, e_{i k}\right)-\frac{\alpha_{i}}{n_{i}} \sum_{k=1}^{n_{i}} \operatorname{cov}\left(e_{i k}, e_{i j^{\prime}}\right) \\
& +\alpha_{i}^{2} \frac{1}{n_{i}^{2}} \sum_{k=1}^{n_{i}} \sum_{l=1}^{n_{i}} \operatorname{cov}\left(e_{i k}, e_{i l}\right) \\
& =-\frac{\alpha_{i}}{n_{i}} \psi_{i j}-\frac{\alpha_{i}}{n_{i}} \psi_{i j^{\prime}}+\frac{\alpha_{i}^{2}}{n_{i}^{2}} \sum_{k=1}^{n_{i}} \psi_{i k} \\
& =-\frac{\alpha_{i}}{n_{i}}\left(\psi_{i j}+\psi_{i j^{\prime}}\right)+\frac{\alpha_{i}^{2}}{n_{i}^{2}} \sum_{k=1}^{n_{i}} \psi_{i k} .
\end{aligned}
$$

2. If $i=i^{\prime}$ and $j=j^{\prime}$

$$
\begin{aligned}
\operatorname{cov}\left(e_{i j}^{\star}, e_{i^{\prime} j^{\prime}}^{\star}\right) & =\operatorname{var}\left(e_{i j}, e_{i j}-\alpha_{i} \bar{e}_{i}\right) \\
& =\operatorname{var}\left(e_{i j}\right)+\alpha_{i}^{2} \operatorname{var}\left(\bar{e}_{i}\right)-2 \alpha_{i} \operatorname{cov}\left(e_{i j}, \bar{e}_{i}\right) \\
& =\left(1-2 \frac{\alpha_{i}}{n_{i}}\right) \psi_{i j}+\frac{\alpha_{i}^{2}}{n_{i}^{2}} \sum_{k=1}^{n_{i}} \psi_{i k} .
\end{aligned}
$$

3. If $i \neq i^{\prime}$

$$
\operatorname{cov}\left(e_{i j}^{\star}, e_{i^{\prime} j^{\prime}}^{\star}\right)=0
$$


Let $\mathbf{e}^{\star}=\left(\mathbf{e}_{1}^{\star}, \cdots, \mathbf{e}_{m}^{\star}\right)^{T}$, where $\mathbf{e}_{i}^{\star}=\left(e_{i 1}^{\star}, e_{i 1}^{\star}, \cdots, e_{i n_{i}}^{\star}\right)^{T}$. Then, $\mathbf{e}^{\star}$ has a block diagonal covariance matrix:

$$
\text { where } V_{i}^{\star}=\left[\begin{array}{cccc}
v\left(e_{i 1}^{\star}\right) & \operatorname{cov}\left(e_{i 1}^{\star}, e_{i 2}^{\star}\right) & \ldots & \operatorname{cov}\left(e_{i 1}^{\star}, e_{i n_{i}}^{\star}\right) \\
\operatorname{cov}\left(e_{i 2}^{\star}, e_{i 1}^{\star}\right) & v\left(e_{i 2}^{\star}\right) & \ldots & \operatorname{cov}\left(e_{i 2}^{\star}, e_{i n_{i}}^{\star}\right) \\
\vdots & \vdots & \ddots & \vdots \\
\operatorname{cov}\left(e_{i n_{i}}^{\star}, e_{i 1}^{\star}\right) & \ldots & \ldots & v\left(e_{i n_{i}}^{\star}\right)
\end{array}\right] .
$$

Using matrix element notation, $V_{i}^{\star}$ can be expressed as follows:

$$
V_{i}^{\star}=\left\{c_{i j k}^{\star}\right\}_{j=1}^{n_{i}} \begin{aligned}
& n_{i} \\
& k=1
\end{aligned},
$$

where $c_{i j k}^{\star}=\delta_{k i j}-\frac{\alpha_{i}}{n_{i}}\left(\psi_{i j}+\psi_{i k}\right)+\frac{\alpha_{i}^{2}}{n_{i}^{2}} \sum_{l=1}^{n_{i}} \psi_{i l}$ with

$$
\delta_{i j k}=\left\{\begin{array}{lll}
1 & \text { if } & j=k \\
0 & \text { if } & j \neq k
\end{array} .\right.
$$

It should be noted that the transformed data depends on the unknown $\rho$. Rao et al. (1993) used Henderson's method Henderson (1953) to estimate $\rho$. We will discuss this in further detail in Section 5.3.3.

\subsubsection{New variable selection criterion based on $\theta^{\star}$}

The desired variable selection criterion can be seen as a function of MST and MSE of the transformed data, $\mathbf{y}^{\star}=\left(\mathbf{y}_{1}^{\star}, \cdots, \mathbf{y}_{m}^{\star}\right)^{T}$, where $\mathbf{y}_{i}^{\star}=\left(y_{i 1}^{\star}, \cdots, y_{i n_{i}}^{\star}\right)^{T}$. The goal is to obtain a good approximation to that criterion based on $\boldsymbol{\theta}^{\star}$, instead of 
$\mathbf{y}^{\star}$. We first derive the SSE and SST of the transformed data.

$$
\begin{aligned}
S S E & =\mathbf{y}^{\star T}\left(\mathbf{I}-\mathbf{P}^{\star}\right) \mathbf{y}^{\star} \\
& =\left(\boldsymbol{\theta}^{\star}+\mathbf{e}^{\star}\right)^{T}\left(\mathbf{I}-\mathbf{P}^{\star}\right)\left(\boldsymbol{\theta}^{\star}+\mathbf{e}^{\star}\right) \\
& =\boldsymbol{\theta}^{\star T}\left(\mathbf{I}-\mathbf{P}^{\star}\right) \boldsymbol{\theta}^{\star}+\boldsymbol{\theta}^{\star T}\left(\mathbf{I}-\mathbf{P}^{\star}\right) \mathbf{e}^{\star}+\mathbf{e}^{\star T}\left(\mathbf{I}-\mathbf{P}^{\star}\right) \boldsymbol{\theta}^{\star}+\mathbf{e}^{\star T}\left(\mathbf{I}-\mathbf{P}^{\star}\right) \mathbf{e}^{\star} \\
& =\boldsymbol{\theta}^{\star T}\left(\mathbf{I}-\mathbf{P}^{\star}\right) \boldsymbol{\theta}^{\star}+2 \boldsymbol{\theta}^{\star T}\left(\mathbf{I}-\mathbf{P}^{\star}\right) \mathbf{e}^{\star}+\mathbf{e}^{\star T}\left(\mathbf{I}-\mathbf{P}^{\star}\right) \mathbf{e}^{\star},
\end{aligned}
$$

where $\mathbf{P}^{\star}=\mathbf{X}^{\star}\left(\mathbf{X}^{\star T} \mathbf{X}^{\star}\right)^{-1} \mathbf{X}^{\star T}, \mathbf{X}^{\star}=\left(\mathbf{x}_{1}^{\star}, \cdots, \mathbf{x}_{m}^{\star}\right)^{T}$ and $\mathbf{x}_{i}^{\star}=\left(\mathbf{x}_{i 1}^{\star}, \cdots, \mathbf{x}_{i n_{i}}^{\star}\right)^{T}$.

$$
\begin{aligned}
S S T & =\mathbf{y}^{\star T}\left(\mathbf{I}-\mathbf{M}^{\star}\right) \mathbf{y}^{\star} \\
& =\left(\boldsymbol{\theta}^{\star}+\mathbf{e}^{\star}\right)^{T}\left(\mathbf{I}-\mathbf{M}^{\star}\right)\left(\boldsymbol{\theta}^{\star}+\mathbf{e}^{\star}\right) \\
& =\boldsymbol{\theta}^{\star T}\left(\mathbf{I}-\mathbf{M}^{\star}\right) \boldsymbol{\theta}^{\star}+\boldsymbol{\theta}^{\star T}\left(\mathbf{I}-\mathbf{M}^{\star}\right) \mathbf{e}^{\star}+\mathbf{e}^{\star T}\left(\mathbf{I}-\mathbf{M}^{\star}\right) \boldsymbol{\theta}^{\star}+\mathbf{e}^{\star T}\left(\mathbf{I}-\mathbf{M}^{\star}\right) \mathbf{e}^{\star} \\
& =\boldsymbol{\theta}^{\star T}\left(\mathbf{I}-\mathbf{M}^{\star}\right) \boldsymbol{\theta}^{\star}+2 \boldsymbol{\theta}^{\star T}\left(\mathbf{I}-\mathbf{M}^{\star}\right) \mathbf{e}^{\star}+\mathbf{e}^{\star T}\left(\mathbf{I}-\mathbf{M}^{\star}\right) \mathbf{e}^{\star},
\end{aligned}
$$

where $\mathbf{M}^{\star}=\frac{1}{n} \mathbf{1 1}^{T}$ with $n=\sum_{i=1}^{m} n_{i}$.

To find the approximation with respect to $\boldsymbol{\theta}^{\star}$, we check the property of MSE and MST conditioned on $\boldsymbol{\theta}^{\star}$. We can show that

$$
\begin{gathered}
E\left(M S E \mid \boldsymbol{\theta}^{\star}\right)=M S E_{\boldsymbol{\theta}^{\star}}+\frac{1}{n-p} \sum_{i=1}^{m} \sum_{j, k=1}^{n_{i}}\left(\delta_{i j k}-h_{i j k}^{\star}\right) c_{i j k}^{\star}, \\
E\left(M S T \mid \boldsymbol{\theta}^{\star}\right)=M S T_{\boldsymbol{\theta}^{\star}}+\frac{1}{n} \sum_{i=1}^{m} \sum_{j, k=1}^{n_{i}} c_{i j k}^{\star},
\end{gathered}
$$


where $M S E_{\boldsymbol{\theta}^{\star}}$ and $M S T_{\boldsymbol{\theta}^{\star}}$ are obtained from MSE and MST, respectively, when we replace $\mathbf{y}$ with $\boldsymbol{\theta}^{\star}, h_{i j k}=\mathbf{x}_{i j}^{\star T}\left(\mathbf{X}^{\star T} \mathbf{X}^{\star}\right)^{-1} \mathbf{x}_{i k}^{\star}, c_{i j k}^{\star}$ is given in (5.13) and

$$
\delta_{i j k}=\left\{\begin{array}{lll}
1 & \text { if } & j=k \\
0 & \text { if } & j \neq k
\end{array}\right.
$$

Proof of (5.16) and (5.17) are given in Section 5.6.

For asymptotic approximation, we adopt the setup where the number of sampled areas $m$ increases to infinity, and the number of sampled subareas within each sampled area is bounded above by some constant $n_{0}$, i.e., $0<n_{i} \leq n_{0}$ for all $i$. We assume that the sampling variance $\psi_{i j}$ are uniformly bounded by some finite constant $\psi_{0}$, i.e., $\psi_{i j} \leq \psi_{0}<\infty$ for all $i$ and $j$. Under this setup we can show that

$$
\begin{aligned}
& \operatorname{Var}\left(M S E \mid \boldsymbol{\theta}^{\star}\right)=O\left(\frac{1}{m}\right), \\
& \operatorname{Var}\left(M S T \mid \boldsymbol{\theta}^{\star}\right)=O\left(\frac{1}{m}\right) .
\end{aligned}
$$

The proof of (5.18) can be found in Section 5.6. Using the Chebyshev's inequality and (5.18) we can show that as $m \rightarrow \infty$ (or equivalently as $n \rightarrow \infty$ )

$$
\begin{gathered}
M S E-E\left(M S E \mid \boldsymbol{\theta}^{\star}\right)=o_{p}(1), \\
M S T-E\left(M S T \mid \boldsymbol{\theta}^{\star}\right)=o_{p}(1) .
\end{gathered}
$$

The derivation of the above equations is shown in Section 5.6. 
Thus, by (5.16), (5.17) and (5.19) we have:

$$
\begin{aligned}
& M S E_{\theta^{\star}}=M S E-\frac{1}{n-p} \sum_{i=1}^{m} \sum_{j, k=1}^{n_{i}}\left(\delta_{i j k}-h_{i j k}^{\star}\right) c_{i j k}^{\star}+o_{p}(1), \\
& M S T_{\boldsymbol{\theta}^{\star}}=M S T-\frac{1}{n} \sum_{i=1}^{m} \sum_{j, k=1}^{n_{i}} c_{i j k}^{\star}+o_{p}(1) .
\end{aligned}
$$

This suggests the following estimators of $M S E_{\theta^{\star}}$ and $M S T_{\theta^{\star}}$

$$
\begin{aligned}
& \widehat{M S E}_{\boldsymbol{\theta}^{\star}}=M S E-\frac{1}{n-p} \sum_{i=1}^{m} \sum_{j, k=1}^{n_{i}}\left(\delta_{i j k}-h_{i j k}^{\star}\right) c_{i j k}^{\star}, \\
& \widehat{M S T}_{\boldsymbol{\theta}^{\star}}=M S T-\frac{1}{n} \sum_{i=1}^{m} \sum_{j, k=1}^{n_{i}} c_{i j k}^{\star} .
\end{aligned}
$$

Clearly, we have:

$$
\begin{aligned}
& \widehat{M S E}_{\boldsymbol{\theta}^{\star}}-M S E_{\boldsymbol{\theta}^{\star}}=o_{p}(1), \\
& \widehat{M S T}_{\boldsymbol{\theta}^{\star}}-M S T_{\boldsymbol{\theta}^{\star}}=o_{p}(1) .
\end{aligned}
$$

By the continuous mapping theorem, for a function $f: \mathbb{R} \rightarrow \mathbb{R}$, we have:

$$
f\left(\widehat{M S E}_{\boldsymbol{\theta}^{\star}}, \widehat{M S T}_{\boldsymbol{\theta}^{\star}}\right)-f\left(M S E_{\boldsymbol{\theta}^{\star}}, M S T_{\boldsymbol{\theta}^{\star}}\right)=o_{p}(1) .
$$

The $f\left(\widehat{M S E}_{\boldsymbol{\theta}^{\star}}, \widehat{M S T}_{\boldsymbol{\theta}^{\star}}\right)$ may go outside of the acceptable range of the desired criterion. Hence, we apply a positive approximation to $\widehat{M S E}_{\boldsymbol{\theta}^{\star}}$ and $\widehat{M S T}_{\boldsymbol{\theta}^{\star}}$. We define a function $h(a, b)$ as suggested by Chatterjee and Lahiri (2007)

$$
h(a, b)=\frac{2 a}{1+\exp \left(\frac{2 b}{a}\right)} .
$$


We apply (5.23) to (5.20), and define the "new" variable selection criterion as $f\left(\widehat{M S E}_{\boldsymbol{\theta}^{\star}, h}, \widehat{M S T}_{\boldsymbol{\theta}^{\star}, h}\right)$, where

$$
\begin{aligned}
& \widehat{M S E}_{\boldsymbol{\theta}^{\star}, h}=h\left(M S E_{\boldsymbol{\theta}^{\star}}, \phi_{1}\right), \\
& \widehat{M S T}_{\boldsymbol{\theta}^{\star}, h}=h\left(M S T_{\boldsymbol{\theta}^{\star}}, \phi_{2}\right),
\end{aligned}
$$

where $\phi_{1}=\frac{1}{n-p} \sum_{i=1}^{m} \sum_{j, k=1}^{n_{i}}\left(\delta_{i j k}-h_{i j k}^{\star}\right) c_{i j k}^{\star}$ and $\phi_{2}=\frac{1}{n} \sum_{i=1}^{m} \sum_{j, k=1}^{n_{i}} c_{i j k}^{\star}$. Approximation (5.23) is always positive and maintains the same asymptotic level discussed above, so we have

$$
f\left(\widehat{M S E}_{\boldsymbol{\theta}^{\star}, h}, \widehat{M S T}_{\boldsymbol{\theta}^{\star}, h}\right)-f\left(M S E_{\boldsymbol{\theta}^{\star}}, M S T_{\boldsymbol{\theta}^{\star}}\right)=o_{p}(1)
$$

Alternatively, a truncated form of the selection criterion can be considered, $f\left(\widehat{M S E}_{\theta^{\star}, \text { trun }}, \widehat{M S T}_{\theta^{\star}, \text { trun }}\right)$, where

$$
\begin{aligned}
& \widehat{M S E}_{\theta^{\star}, \text { trun }}=\left\{\begin{array}{lll}
\widehat{M S E}_{\theta^{\star}} & \text { if } & \widehat{M S E}_{\theta^{\star}} \geq 0 \\
\widehat{M S E}_{\boldsymbol{\theta}^{\star}, h} & \text { otherwise }
\end{array}\right. \\
& \widehat{M S T}_{\theta^{\star}, \text { trun }}=\left\{\begin{array}{lll}
\widehat{M S T}_{\theta^{\star}} & \text { if } & \widehat{M S T}_{\theta^{\star}} \geq 0 \\
\widehat{M S T}_{\boldsymbol{\theta}^{\star}, h} & \text { otherwise }
\end{array}\right.
\end{aligned}
$$

To sum up, for a dataset following a two-fold subarea level model, we first transform the variable of interest $y_{i j}$ (i.e., direct subarea estimates) and covariates $x_{i j_{k}}$ using Fuller-Battese transformation, and obtain $y_{i j}^{\star}$ and $x_{i j_{k}}^{\star}$. Then, for a desired criterion $f(M S E, M S T)$, we form $f\left(\widehat{M S E}_{\boldsymbol{\theta}^{\star}, h}, \widehat{M S T}_{\boldsymbol{\theta}^{\star}, h}\right)$ in (5.24) or $f\left(\widehat{M S E}_{\theta^{\star}, \text { trun }}, \widehat{M S T}_{\theta^{\star}, \text { trun }}\right)$ in $(5.25)$. 


\subsubsection{Unknown $\rho$}

The Fuller-Battese transformation used in the first step of the adjustment depends on $\rho$. In practice, $\rho$ is unknown and must be estimated from the data. Torabi and Rao (2014) described the process of estimation of variance components $\sigma_{v}^{2}$ and $\sigma_{u}^{2}$, using iterative moment methods. More precisely, they extended the iterative moment method of Fay and Herriot (Fay and Herriot, 1979) to the subarea level model (5.8).

Equation (5.8) is a special case of a general linear mixed model with block diagonal covariance structure. Let $\mathbf{y}_{i}=\left(\hat{\theta}_{i 1}, \cdots, \hat{\theta}_{i n_{i}}\right)^{T}$ and $\mathbf{e}_{i}=\left(e_{i 1}, \cdots, e_{i n_{i}}\right)^{T}$, (5.8) then can be written as:

$$
\mathbf{y}_{i}=\mathbf{X}_{i} \boldsymbol{\beta}+\mathbf{Z}_{i} \mathbf{v}_{i}+\mathbf{e}_{i}, \quad i=1, \ldots, m
$$

where $\mathbf{X}_{i}$ is the $n_{i} \times p$ matrix with rows $\mathbf{x}_{i j}^{T}, \mathbf{Z}_{i}=\left(\mathbf{1}_{n i} \mid \mathbf{I}_{n i}\right)$, and $\mathbf{v}_{i}=\left(v_{i}, \mathbf{u}_{i}^{T}\right)^{T}$ with $\mathbf{u}_{i}=\left(u_{i 1}, \cdots, u_{i, n_{i}}\right)^{T}$. Let $\operatorname{cov}\left(\mathbf{y}_{i}\right)=\mathbf{V}_{i}=\sigma_{v}^{2} \mathbf{1}_{n_{i}} \mathbf{1}_{n_{i}}^{T}+\operatorname{diag}\left(\sigma_{u}^{2}+\psi_{i 1}, \cdots, \sigma_{u}^{2}+\psi_{i n_{i}}\right)$, using weighted residual sum of squares, we have:

$$
E\left\{\sum_{i=1}^{m}\left(\mathbf{y}_{i}-\mathbf{X}_{i} \tilde{\boldsymbol{\beta}}\right)^{T} \mathbf{V}_{i}^{-1}\left(\mathbf{y}_{i}-\mathbf{X}_{i} \tilde{\boldsymbol{\beta}}\right)\right\}=n-p
$$

where $\tilde{\boldsymbol{\beta}}=\left(\sum_{i=1}^{m} \mathbf{X}_{i}^{T} \mathbf{V}_{i}^{-1} \mathbf{X}_{i}\right)^{-1}\left(\sum_{i=1}^{m} \mathbf{X}_{i}^{T} \mathbf{V}_{i} \mathbf{y}_{i}\right)$ is the WLS estimator of $\boldsymbol{\beta}$. The following estimating equation is formed using the method of moments:

$$
\sum_{i=1}^{m}\left(\mathbf{y}_{i}-\mathbf{X}_{i} \tilde{\boldsymbol{\beta}}\right)^{T} \mathbf{V}_{i}^{-1}\left(\mathbf{y}_{i}-\mathbf{X}_{i} \tilde{\boldsymbol{\beta}}\right)=n-p .
$$


Taking the average of model (5.8) over $j$ we get

$$
\begin{aligned}
\bar{y}_{i} & =\overline{\mathbf{x}}_{i}^{T} \boldsymbol{\beta}+v_{i}+\bar{u}_{i}+\bar{e}_{i}, \quad, i=1, \cdots, m, \\
& =\overline{\mathbf{x}}_{i}^{T} \boldsymbol{\beta}+\epsilon_{i},
\end{aligned}
$$

where $\bar{y}_{i}, \overline{\mathbf{x}}_{i}, \bar{u}_{i}$ and $\bar{e}_{i}$ are averages of $y_{i j}, \mathbf{x}_{i j}, u_{i j}$ and $e_{i j}$ over $j=1, \cdots, n_{i}$, and $\epsilon_{i} \sim^{\text {ind }} N\left\{0, \tilde{\delta}_{i}^{2}=n_{i}^{-1}\left(n_{i} \sigma_{v}^{2}+\sigma_{u}^{2}+\psi_{i j}\right)\right\}$. Next, the WLS estimator of $\boldsymbol{\beta}$ associated with (5.29) should be obtained as $\tilde{\boldsymbol{\beta}}^{*}=\left(\overline{\mathbf{x}}_{i} \overline{\mathbf{x}}_{i}^{T} / \tilde{\delta}_{i}\right)^{-1}\left(\overline{\mathbf{x}}_{i} \overline{\mathbf{y}}_{i} / \tilde{\delta}_{i}\right)$, as well as the associated weighted residual sum of squares $\sum_{i=1}^{m}\left(\bar{y}_{i}-\overline{\mathbf{x}}_{i}^{T} \tilde{\boldsymbol{\beta}}^{*}\right)^{2} / \tilde{\delta}_{i}=m-p$ with expectation equal to $m-p$. Then, we use the method of moments to form the estimating equation

$$
\sum_{i=1}^{m}\left(\bar{y}_{i}-\overline{\mathbf{x}}_{i}^{T} \tilde{\boldsymbol{\beta}}^{*}\right)^{2} / \tilde{\delta}_{i}=m-p
$$

The estimators of $\tilde{\sigma}_{v}^{2}$ and $\tilde{\sigma}_{u}^{2}$ are obtained by solving (5.27) and (5.30) iteratively. Eventually, to avoid negative values, $\hat{\sigma}_{v}^{2}$ and $\hat{\sigma}_{u}^{2}$ are defined as

$$
\begin{aligned}
& \hat{\sigma}_{v}^{2}=\max \left(0, \tilde{\sigma}_{v}^{2}\right) \\
& \hat{\sigma}_{u}^{2}=\max \left(0, \tilde{\sigma}_{u}^{2}\right) .
\end{aligned}
$$

Once we obtain $\hat{\sigma}_{v}^{2}$ and $\hat{\sigma}_{u}^{2}$ from (5.31), we can estimate $\rho$ by

$$
\hat{\rho}=\frac{\hat{\sigma}_{v}^{2}}{\hat{\sigma}_{v}^{2}+\hat{\sigma}_{u}^{2}} .
$$

The challenge in a variable selection problem is in defining $\mathbf{X}_{i}$ in (5.26). Ideally, $\rho$ in Fuller-Battese should be estimated using the "true" model, which is not known in practice. One solution is to estimate $\rho$ from the full model (i.e., including all 
covariates in the model) to preserve the consistency of $\hat{\rho}$. This is not a perfect solution as the true model may be different from the full model, but it should be noted that the full model always includes the true model, so by this approach $\hat{\rho}$ is estimated based a correct model and not necessarily an optimal model.

\subsection{Simulation study}

A simulation study is conducted to examine the performance of the proposed adjustment to selection criteria. The following model was considered:

$$
\begin{aligned}
& y_{i j}=\beta_{0} x_{i j_{0}}+\beta_{1} x_{i j_{1}}+\beta_{2} x_{i j_{2}}+\beta_{3} x_{i j_{3}}+\beta_{4} x_{i j_{4}}+v_{i}+u_{i j}+e_{i j}, \\
& i=1, \ldots, 30, \quad j=1, \ldots, n_{i} .
\end{aligned}
$$

We set the number of subareas $n_{i}$ in each area equal to 4 . In total, there are 120 subareas in each sample. For simplicity, we considered the balanced subarea model with common mean and error variances, $\psi_{i j}=\psi_{i}$. We used the following distributions for generating $x_{i j_{k}}, \quad k=1, \cdots, 4$ :

$$
\begin{aligned}
& x_{i j_{1}} \sim \log \text {-normal }(0.5,1), \\
& x_{i j_{2}} \sim \operatorname{Gamma}(1.5,0.8) \text { where } \operatorname{Gamma}(\alpha, \beta) \text { denotes a gamma distribution } \\
& \text { with shape parameter } \alpha \text { and scale parameter } \beta, \\
& x_{i j_{3}} \sim N(0,1), \\
& x_{i j_{4}} \sim \operatorname{Gamma}(0.7,0.1) .
\end{aligned}
$$$$
\text { with shape parameter } \alpha \text { and scale parameter } \beta \text {, }
$$

The subarea random effect, $u_{i j}$, are distributed as $N(0,1)$ and $e_{i j} \sim N\left(0, \psi_{i}\right)$, where $\sqrt{\psi_{i}}$ are generated from $\operatorname{Gamma}(0.5,1)+0.5$. The $v_{i}$ 's are distributed as 
$N\left(0, \sigma_{v}^{2}\right)$ independent of $e_{i j}$ and $u_{i j}$. The value of $x_{i j 0}$ is 1 for all units, $i=1, \ldots, 30$, and $j=1, \ldots, n_{i}=4$.

We consider the selection of variables $x_{i j k}$ for $k=1, \cdots, 4$. There are 15 possible models in total. Each model is denoted by a subset of $(0,1,2,3,4)$ that contains the indices of $x_{i j_{k}}$ 's in the model. It should be noted that some of the $\beta_{k}$, $k=1, \cdots, 4$ may be zero. We generate 1000 independent set of $y_{i j}$ 's for several values of $\sigma_{v}^{2}$, and estimated the probability of selecting each model using AIC measure and the adjusted $R^{2}$. In each simulation run, we calculate the following measures:

a) $A I C_{\text {naive1 }}$ and $R^{2}(a d j)_{\text {naive } 1}$

The standard AIC and adjusted R-squared measures, that ignore random effects as well as the sampling errors, calculated using $y_{i j}$ an $x_{i j}$ values (i.e., not transformed observations):

$$
\begin{aligned}
& A I C_{\text {naive } 1}=2 p+n \log \left(\frac{S S E}{n}\right), \\
& R^{2}(a d j)_{n a i v e 1}=1-\frac{(n-1) S S E}{(n-p) S S T}
\end{aligned}
$$

where $n$ is the number of subareas, $p$ is the number of covariates, $S S T=$ $\mathbf{y}^{T}\left[\mathbf{1}-n^{-1} \mathbf{1 1}^{T}\right] \mathbf{y}, S S E=\mathbf{y}^{T}[\mathbf{I}-\mathbf{P}] \mathbf{y}$ with $\mathbf{P}=\mathbf{X}\left(\mathbf{X}^{T} \mathbf{X}\right)^{-1} \mathbf{X}^{T}$. Indeed, $A I C_{\text {naive } 1} R^{2}(a d j)_{n a i v e 1}$ are the measures calculated in standard regression packages, which are used by most users.

b) $A I C_{\text {naive2 }}$ and $R^{2}(\operatorname{adj})_{\text {naive } 2}$

These criteria are calculated by naively applying Lahiri and Suntornchost's approximation described in Section 5.2.1. $A I C_{\text {naive } 2}$ and $R^{2}(\operatorname{adj})_{n a i v e 2}$ ignore 
the area effect $v_{i}$ and assume that the underlying linking model is:

$$
\theta_{i j}=\mathbf{x}_{i j}^{T} \boldsymbol{\beta}+u_{i j}
$$

Following Lahiri and Suntornchost (2015), the $A I C_{\text {naive } 2}$ and $R^{2}(\text { adj })_{\text {naive } 2}$ are calculated as follows.

$$
\begin{aligned}
& A I C_{\text {naive } 2}=2 p+n \log \left(\frac{\widehat{S S E}}{n}\right), \\
& R^{2}(\operatorname{adj})_{\text {naive } 2}=1-\frac{(n-1) \widehat{S S E}}{(n-p) \widehat{S S T}},
\end{aligned}
$$

where

$$
\begin{aligned}
& \widehat{S S T}=\mathbf{y}^{T}\left[\mathbf{1}-n^{-1} \mathbf{1}^{T}\right] \mathbf{y}-\frac{n-1}{n} \sum_{i=1}^{m} \sum_{j=1} n_{i} \psi_{i j} \\
& \widehat{S S E}=\mathbf{y}^{T}[\mathbf{I}-\mathbf{P}] \mathbf{y}-\sum_{i=1}^{m} \sum_{j=1}^{n_{i}}\left(1-h_{i i}\right) \psi_{i j},
\end{aligned}
$$

with $h_{i i}=\mathbf{x}_{i j}\left(\mathbf{X}^{T} \mathbf{X}\right)^{-1} \mathbf{x}_{i j}$

c) $A I C_{\text {new }}$ and $R^{2}(\operatorname{adj})_{\text {new }}$

These measures are calculated based on the proposed two-step adjustment, using (5.24).

$$
\begin{aligned}
& A I C_{\text {new }}=2 p+n \log \left(\frac{\widehat{S S E}_{\boldsymbol{\theta}^{\star}, h}}{n}\right) \\
& R^{2}(\operatorname{adj})_{n e w}=1-\frac{(n-1) \widehat{S S E}_{\boldsymbol{\theta}^{\star}, h}}{(n-p) \widehat{S S T}_{\boldsymbol{\theta}^{\star}, h}}
\end{aligned}
$$


where

$$
\begin{aligned}
& \widehat{S S T}_{\boldsymbol{\theta}^{\star}, h}=(n-1) h\left(M S T_{\boldsymbol{\theta}^{\star}}, \frac{1}{n-p} \sum_{i=1}^{m} \sum_{j, k=1}^{n_{i}} c_{i j k}^{\star}\right), \\
& \widehat{S S E}_{\boldsymbol{\theta}^{\star}, h}=(n-p) h\left(M S E_{\boldsymbol{\theta}^{\star}}, \frac{1}{n-p} \sum_{i=1}^{m} \sum_{j, k=1}^{n_{i}}\left(1-h_{i j k}^{\star}\right) c_{i j k}^{\star}\right),
\end{aligned}
$$

with $h(a, b)=\frac{2 a}{1+\exp \left(\frac{2 b}{a}\right)}$.

We tested the model with five different values of $\sigma_{v}^{2}=1,5,10,16,24$, and values of $\boldsymbol{\beta}^{T}$ are taken to be $(2,0,0,4,8),(2,3,0,4,8)$ and $(2,3,5,4,8)$. Models are classified as optimal (or true model), category I (correct model but over-parametrized) and category II (incorrect model).

Results of $A I C_{\text {naive1 }}$ are presented in Table 5.1. In general, the performance of $A I C_{\text {naive } 1}$ is not good for large value of $\sigma_{v}^{2}$. In particular, for the model with $\beta=(2,3,0,4,8)^{T}$, the estimated selection probability of the true model with $\sigma_{v}^{2}=16$ is $51.5 \%$, which means for almost half of the tested datasets $A I C_{\text {naive } 1}$ chose an over-parameterized model $(12 \%)$ or a wrong model $(36.2 \%)$, instead of the true model. As value of $\sigma_{v}^{2}$ increases, the probability of selecting the optimal model decreases. $A I C_{\text {naive } 1}$ performs well for small values of $\sigma_{v}^{2}$, when the optimal model is the full model. For example, for model 3 with $\left.\beta=(2,3,5,4,8)^{T}\right)$, when $\sigma_{v}^{2}=1$, the probability of selecting the optimal model is $99.8 \%$. 
Table 5.1: Probabilities of model selection using $A I C_{\text {naive1 }}$

\begin{tabular}{||ccccccc||}
\hline \hline \multicolumn{7}{|c||}{$\beta=(2,0,0,4,8)^{T}$} \\
\hline Model & Category & $\sigma_{v}^{2}=1$ & $\sigma_{v}^{2}=5$ & $\sigma_{v}^{2}=10$ & $\sigma_{v}^{2}=16$ & $\sigma_{v}^{2}=24$ \\
$(0,3,4)$ & optimal & 71.7 & 61.8 & 52.1 & 42.0 & 38.4 \\
$(0,1,3,4)$ & I & 13.3 & 0.3 & 0.5 & 1.0 & 1.3 \\
$(0,2,3,4)$ & I & 12.5 & 10.7 & 10.8 & 8.9 & 6.4 \\
$(0,1,2,3,4)$ & I & 2.2 & 13.6 & 11.3 & 10.6 & 9.5 \\
$(0,3)$ & II & 0.1 & 8.2 & 17.1 & 24.9 & 32.9 \\
$(0,1,3)$ & II & 0.1 & 1.5 & 2.3 & 4.9 & 4.5 \\
$(0,2,3)$ & II & 0.1 & 1.4 & 3.4 & 5.6 & 5.7 \\
\hline \hline & & & $\beta=(2,3,0,4,8)^{T}$ & & & \\
\hline Model & Category & $\sigma_{v}^{2}=1$ & $\sigma_{v}^{2}=5$ & $\sigma_{v}^{2}=10$ & $\sigma_{v}^{2}=16$ & $\sigma_{v}^{2}=24$ \\
\hline$(0,1,3,4)$ & optimal & 81.4 & 71.7 & 61.1 & 51.5 & 45.4 \\
$(0,1,2,3,4)$ & I & 18.5 & 18.1 & 13.9 & 12.3 & 9.4 \\
$(0,1,3)$ & II & 0.1 & 8.7 & 20.7 & 30.5 & 36.4 \\
$(0,1,2,3)$ & II & 0.0 & 1.5 & 4.3 & 5.7 & 8.7 \\
\hline \hline Model & Category & $\sigma_{v}^{2}=1$ & $\sigma_{v}^{2}=5$ & $\sigma_{v}^{2}=10$ & $\sigma_{v}^{2}=16$ & $\sigma_{v}^{2}=24$ \\
\hline$(0,1,2,3,4)$ & optimal & 99.8 & 91.4 & 75.9 & 63.2 & 53.1 \\
$(0,1,2,3)$ & II & 0.2 & 8.6 & 24.1 & 36.8 & 46.9 \\
\hline \hline
\end{tabular}

We repeat the above process, but this time we calculate $A I C_{\text {naive } 2}$ for all possible combinations. Results of $A I C_{\text {naive2 }}$ are presented in Table 5.2. Overall, the results of $A I C_{\text {naive } 2}$ is more or less similar to $A I C_{\text {naive1 }}$. For some tested models, the estimated probability of selecting the optimal model increases slightly when $A I C_{\text {naive2 }}$ is used. 
Table 5.2: Probabilities of model selection using $A I C_{\text {naive2 }}$

\begin{tabular}{||ccccccc||}
\hline \hline \multicolumn{7}{|c||}{$\beta=(2,0,0,4,8)^{T}$} \\
\hline Model & Category & $\sigma_{v}^{2}=1$ & $\sigma_{v}^{2}=5$ & $\sigma_{v}^{2}=10$ & $\sigma_{v}^{2}=16$ & $\sigma_{v}^{2}=24$ \\
\hline$(0,3,4)$ & optimal & 86.3 & 69.7 & 52.7 & 40.9 & 32.1 \\
$(0,1,3,4)$ & I & 6.6 & 3.7 & 3.3 & 1.2 & 0.2 \\
$(0,2,3,4)$ & I & 5.3 & 4.7 & 3.8 & 4.1 & 1.4 \\
$(0,1,2,3,4)$ & I & 0.6 & 0.4 & 0.4 & 0.3 & 2.6 \\
$(0,3)$ & II & 0.9 & 19.3 & 35.7 & 48.7 & 58.6 \\
$(0,1,3)$ & II & 0.1 & 1.2 & 2.2 & 1.8 & 2.0 \\
$(0,2,3)$ & II & 0.2 & 1.0 & 1.9 & 3.0 & 3.1 \\
\hline \hline Model & Category & $\sigma_{v}^{2}=1$ & $\sigma_{v}^{2}=5$ & $\sigma_{v}^{2}=10$ & $\sigma_{v}^{2}=16$ & $\sigma_{v}^{2}=24$ \\
\hline$(0,1,3,4)$ & optimal & 90.5 & 72.1 & 53.9 & 51.5 & 33.4 \\
$(0,1,2,3,4)$ & I & 9.2 & 6.6 & 3.6 & 12.3 & 2.5 \\
$(0,1,3)$ & II & 0.3 & 20.2 & 40.5 & 30.5 & 60.6 \\
$(0,1,2,3)$ & II & 0.0 & 1.1 & 2.0 & 5.7 & 3.5 \\
\hline \hline Model & Category & $\sigma_{v}^{2}=1$ & $\sigma_{v}^{2}=5$ & $\sigma_{v}^{2}=10$ & $\sigma_{v}^{2}=16$ & $\sigma_{v}^{2}=24$ \\
\hline$(0,1,2,3,4)$ & optimal & 99.5 & 82.7 & 60.6 & 44.5 & 34.4 \\
$(0,1,2,3)$ & II & 0.5 & 17.3 & 39.4 & 55.5 & 65.6 \\
\hline \hline
\end{tabular}

In particular, for model 1 (i.e., $\left.\beta=(2,0,0,4,8)^{T}\right)$ with $\sigma_{v}^{2}=5$ the probability of selecting the optimal model is $69.7 \%$ with $A I C_{\text {naive } 2}$, and is $61.8 \%$ with $A I C_{\text {naive1 }}$.

Table 5.3 represents the results when we use the proposed two-step adjustment to AIC, $A I C_{n e w}$. 
Table 5.3: Probabilities of model selection using $A I C_{\text {new }}$

\begin{tabular}{||ccccccc||}
\hline \hline \multicolumn{7}{|c||}{$\beta=(2,0,0,4,8)^{T}$} \\
\hline Model & Category & $\sigma_{v}^{2}=1$ & $\sigma_{v}^{2}=5$ & $\sigma_{v}^{2}=10$ & $\sigma_{v}^{2}=16$ & $\sigma_{v}^{2}=24$ \\
\hline$(0,3,4)$ & optimal & 93.2 & 89.5 & 90.7 & 89.5 & 91.5 \\
$(0,1,3,4)$ & I & 0.1 & 0.3 & 0.4 & 0.3 & 0.3 \\
$(0,2,3,4)$ & I & 0.2 & 0.1 & 0.1 & 8.1 & 0.1 \\
$(0,3)$ & II & 6.5 & 10.0 & 8.8 & 10.0 & 0.1 \\
$(0,1,3)$ & II & 0.0 & 0.1 & 0.0 & 0.1 & 0.0 \\
\hline \hline Model & Category & $\sigma_{v}^{2}=1$ & $\sigma_{v}^{2}=5$ & $\sigma_{v}^{2}=10$ & $\sigma_{v}^{2}=16$ & $\sigma_{v}^{2}=24$ \\
\hline$(0,1,3,4)$ & optimal & 93.7 & 90.3 & 88.6 & 88.6 & 90.0 \\
$(0,1,2,3,4)$ & I & 0.0 & 0.0 & 0.2 & 0.2 & 0.1 \\
$(0,1,2,3)$ & II & 0.1 & 0.1 & 0.1 & 0.0 & 9.9 \\
$(0,1,3)$ & II & 6.2 & 9.6 & 11.1 & 11.2 & 0.0 \\
\hline \hline & & & $\beta=(2,3,5,4,8)^{T}$ & & & \\
\hline Model & Category & $\sigma_{v}^{2}=1$ & $\sigma_{v}^{2}=5$ & $\sigma_{v}^{2}=10$ & $\sigma_{v}^{2}=16$ & $\sigma_{v}^{2}=24$ \\
\hline$(0,1,2,3,4)$ & optimal & 92.8 & 92.9 & 91.7 & 90.6 & 90.1 \\
$(0,1,2,3)$ & II & 7.2 & 7.1 & 8.3 & 9.4 & 9.9 \\
\hline \hline
\end{tabular}

The probability of selecting the optimal model increases significantly for all models with $A I C_{n e w}$. The improvement is more significant in models with larger values of $\sigma_{v}^{2}$. For instance, the probability of selecting the true model for the model with $\beta=(2,0,0,4,8)^{T}$ and $\sigma_{v}^{2}=24$ increases by $53.1 \%$, when we use $A I C_{\text {new }}$ instead of $A I C_{\text {naive } 1}$, which is offered in standard regression packages (91.5\% vs. $38.4 \%)$.

For the adjusted R-squared criterion, we observed a similar trend. The difference between $R^{2}(a d j)_{\text {naive1 }}, R^{2}(a d j)_{\text {naive } 2}$ is minimal. 
Figure 5.1 shows the the percentage of selecting the optimal model for $\beta=$ $(2,0,0,4,8)^{T}, \beta=(2,3,0,4,8)^{T}$ and $\beta=(2,3,5,4,8)^{T}$. Complete tables of model selection using adjusted R-squared can be found in Section 5.7.

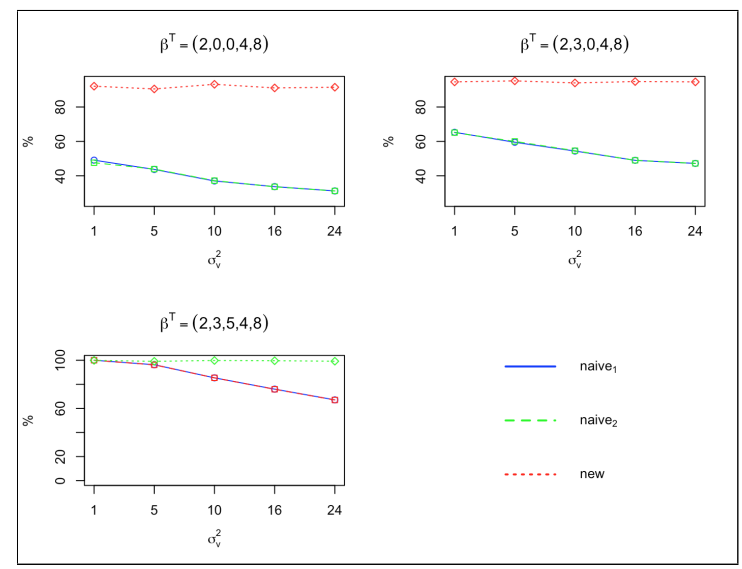

Figure 5.1: Percentage of selecting the optimal model using adjusted R-squared

Overall, $R^{2}(a d j)_{\text {new }}$ performed significantly better than naive versions of the adjusted R-squared. For the model with $\beta=(2,0,0,4,8)^{T}$, the probability of selecting the true model is around $+90 \%$ when the proposed measured is used, while for $R^{2}(a d j)_{\text {naive1 }}, R^{2}(a d j)_{\text {naive2 } 2}$ the percentage is between $30 \%$ to $40 \%$.

\subsection{Summary}

In this chapter, we focused on two-fold subarea models and proposed a two-step adjustment to standard variable selection methods. We first transformed the linking model and the sampling model. Then, we obtained an approximation to the desired regression variable selection criterion using the transformed linking model. We studied the properties of the new selection criterion and showed that the approximation error of the proposed method converges to zero, when the number of areas $(m)$ increases, assuming that the number of subareas, $n_{i}$, is 
bounded. Using a Monte Carlo simulation, we assessed the performance of the new selection criterion. The simulation results suggest that the two-step adjustment increases the chance of selecting the optimal model. It was shown that for fixed $\sigma_{u}^{2}$ the probability of selecting the optimal model decreases as $\sigma_{v}^{2}$ increases. For some models, applying naively Lahiri and Suntornchost's approximation improved the results slightly compared to the naive version of the criterion offered in standard regression packages. The improvement is much more significant when we used the proposed two-step adjustment for models with larger value of $\sigma_{v}^{2}$.

\subsection{Proofs}

In this section, we provide more details for some of the equations in Section 5.3.

\subsubsection{Derivation of (5.16) and (5.17)}

Using (5.14), we have

$$
M S E=\frac{S S E}{n-p}=\frac{1}{n-p}\left\{\boldsymbol{\theta}^{\star T}\left(\mathbf{I}-\mathbf{P}^{\star}\right) \boldsymbol{\theta}^{\star}+2 \boldsymbol{\theta}^{\star T}\left(\mathbf{I}-\mathbf{P}^{\star}\right) \mathbf{e}^{\star}+\mathbf{e}^{\star T}\left(\mathbf{I}-\mathbf{P}^{\star}\right) \mathbf{e}^{\star}\right\} .
$$

Hence,

$$
\begin{aligned}
E\left(M S E \mid \boldsymbol{\theta}^{\star}\right) & =\frac{1}{n-p}\left\{E\left[\boldsymbol{\theta}^{\star T}\left(\mathbf{I}-\mathbf{P}^{\star}\right) \boldsymbol{\theta}^{\star}+2 \boldsymbol{\theta}^{\star T}\left(\mathbf{I}-\mathbf{P}^{\star}\right) \mathbf{e}^{\star}+\mathbf{e}^{\star T}\left(\mathbf{I}-\mathbf{P}^{\star}\right) \mathbf{e}^{\star} \mid \boldsymbol{\theta}^{\star}\right]\right\} \\
& =\frac{1}{n-p}\left\{S S E_{\theta^{\star}}+2 E\left[\boldsymbol{\theta}^{\star T}\left(\mathbf{I}-\mathbf{P}^{\star}\right) \mathbf{e}^{\star} \mid \boldsymbol{\theta}^{\star}\right]+E\left[\mathbf{e}^{\star T}\left(\mathbf{I}-\mathbf{P}^{\star}\right) \mathbf{e}^{\star} \mid \boldsymbol{\theta}^{\star}\right]\right\} \\
& =M S E_{\theta^{\star}}+0+\frac{1}{n-p} E\left[\mathbf{e}^{\star T}\left(\mathbf{I}-\mathbf{P}^{\star}\right) \mathbf{e}^{\star}\right] .
\end{aligned}
$$

$E\left[\mathbf{e}^{\star T}\left(\mathbf{I}-\mathbf{P}^{\star}\right) \mathbf{e}^{\star}\right]$ can be derived using the following theorem: 
Theorem 1 Let $y$ be a random vector and $A$ be a symmetric matrix. If $E[\mathbf{y}]=\boldsymbol{\mu}$ and $V[\mathbf{y}]=\mathbf{V}$, then $E\left[\mathbf{y}^{T} \mathbf{A y}\right]=\operatorname{tr}(\mathbf{A V})+\boldsymbol{\mu}^{T} \mathbf{A} \boldsymbol{\mu}$.

In our notation, $\boldsymbol{\mu}=E\left(\mathbf{e}^{\star}\right)=\mathbf{0}, V[\mathbf{y}]=V\left(\mathbf{e}^{\star}\right)=\operatorname{diag}\left(V_{i}^{\star}\right)_{1 \leq i \leq m}$ and $\mathbf{A}=\mathbf{I}-\mathbf{P}^{\star}$, which is a symmetric matrix. So, $E\left[\mathbf{e}^{\star T}\left(\mathbf{I}-\mathbf{P}^{\star}\right) \mathbf{e}^{\star}\right]$ is equal to

$$
\begin{aligned}
\operatorname{tr}\left[\left(\mathbf{I}-\mathbf{P}^{\star}\right) \operatorname{diag}\left(V_{i}^{\star}\right)\right] & =\sum_{i=1}^{m} \operatorname{tr}\left(\left[I_{n_{i}}-\mathbf{x}_{i}^{\star}\left(\mathbf{X}^{\star T} \mathbf{X}^{\star}\right)^{-1} \mathbf{x}_{i}^{\star T}\right] V_{i}^{\star}\right) \\
& =\sum_{i=1}^{m} \sum_{j, k=1}^{n_{i}}\left(\delta_{i j k}-h_{i j k}^{\star}\right) c_{i j k}^{\star},
\end{aligned}
$$

where

$$
\delta_{i j k}=\left\{\begin{array}{lll}
1 & \text { if } & j=k \\
0 & \text { if } & j \neq k
\end{array},\right.
$$

$h_{i j k}=\mathbf{x}_{i j}^{\star}\left(\mathbf{X}^{\star T} \mathbf{X}^{\star}\right)^{-1} \mathbf{x}_{i k}^{\star T}$ and $c_{i j k}^{\star}$ is given in (5.13).

Hence, $E\left(M S E \mid \boldsymbol{\theta}^{\star}\right)$ can be expressed as

$$
E\left(M S E \mid \boldsymbol{\theta}^{\star}\right)=M S E_{\boldsymbol{\theta}^{\star}}+\frac{1}{n-p} \sum_{i=1}^{m} \sum_{j, k=1}^{n_{i}}\left(\delta_{i j k}-h_{i j k}^{\star}\right) c_{i j k}^{\star} .
$$

Alternatively, we can express $\operatorname{tr}\left[\left(\mathbf{I}-\mathbf{P}^{\star}\right) \operatorname{diag}\left(V_{i}^{\star}\right)\right]$ in matrix form. Let $\mathbf{e}^{\star}=$ $\left(\mathbf{e}_{1}^{\star}, \cdots, \mathbf{e}_{m}^{\star}\right)^{T}$, where $\mathbf{e}_{i}^{\star}=\left(e_{i 1}^{\star}, \cdots, e_{i n_{i}}^{\star}\right)^{T}=\left(e_{i 1}-\bar{e}_{i}, \cdots, e_{i n_{i}}-\bar{e}_{i}\right)^{T}$. So, in matrix form we have:

$$
\mathbf{e}^{\star}=\mathbf{e}-\mathbf{Q e}=(\mathbf{I}-\mathbf{Q}) \mathbf{e},
$$


where $\mathbf{Q}=\left[\begin{array}{cccc}\frac{\alpha_{1}}{n_{1}} \mathbf{1}_{n_{1} \times n_{1}} & \mathbf{0} & \ldots & \mathbf{0} \\ \vdots & \vdots & \frac{\alpha_{i}}{n_{i}} \mathbf{1}_{n_{i} \times n_{i}} & \vdots \\ \mathbf{0} & \mathbf{0} & \ldots & \frac{\alpha_{m}}{n_{m}} \mathbf{1}_{n_{m} \times n_{m}}\end{array}\right]_{n_{\times} n}$.

Evidently,

$$
\begin{aligned}
V\left(\mathbf{e}^{\star}\right) & =V[(\mathbf{I}-\mathbf{Q}) \mathbf{e}] \\
& =(\mathbf{I}-\mathbf{Q}) V(\mathbf{e})(\mathbf{I}-\mathbf{Q})^{T}
\end{aligned}
$$

Using (5.33) and Theorem 1, we have:

$$
\left.E\left(M S E \mid \boldsymbol{\theta}^{\star}\right)=M S E_{\boldsymbol{\theta}^{\star}}+\operatorname{tr}\left[\left(\mathbf{I}-\mathbf{P}^{\star}\right)(\mathbf{I}-\mathbf{Q}) V(\mathbf{e})(\mathbf{I}-\mathbf{Q})^{T}\right)\right] .
$$

Equations (5.34) and (5.16) are equivalent, however (5.34) has some advantages computationally.

Similarly, we can derive $E\left(M S T \mid \boldsymbol{\theta}^{\star}\right)$.

$$
\begin{aligned}
E\left(M S T \mid \boldsymbol{\theta}^{\star}\right)= & \frac{1}{n-1}\left\{E\left[\boldsymbol{\theta}^{\star T}\left(\mathbf{I}-\mathbf{M}^{\star}\right) \boldsymbol{\theta}^{\star}+2 \mathbf{e}^{\star T}\left(\mathbf{I}-\mathbf{M}^{\star}\right) \boldsymbol{\theta}^{\star}+\mathbf{e}^{\star T}\left(\mathbf{I}-\mathbf{M}^{\star}\right) \mathbf{e}^{\star} \mid \boldsymbol{\theta}^{\star}\right]\right\} \\
& =M S T_{\theta^{\star}}+\frac{1}{n-1} E\left[\left(\mathbf{e}^{\star T}\left(\mathbf{I}-\mathbf{M}^{\star}\right) \mathbf{e}^{\star}\right)\right] .
\end{aligned}
$$

Using Theorem 1, we have:

$$
\begin{aligned}
E\left[\mathbf{e}^{\star T}\left(\mathbf{I}-\mathbf{M}^{\star}\right) \mathbf{e}^{\star}\right] & =\operatorname{tr}\left[\left(\mathbf{I}-\mathbf{M}^{\star}\right) \operatorname{diag}\left(V_{i}^{\star}\right)\right] \\
& =\sum_{i=1}^{m} \sum_{j, k=1}^{n_{i}}\left(1-\frac{1}{n}\right) c_{i j k}^{\star} .
\end{aligned}
$$


So, $E\left(M S T \mid \boldsymbol{\theta}^{\star}\right)$ can be written as:

$$
E\left(M S T \mid \boldsymbol{\theta}^{\star}\right)=M S T_{\boldsymbol{\theta}^{\star}}+\frac{1}{n} \sum_{i=1}^{m} \sum_{j, k=1}^{n_{i}} c_{i j k}^{\star} .
$$

The above equation can also be expressed in matrix form as follows

$$
\left.E\left(M S T \mid \boldsymbol{\theta}^{\star}\right)=M S T_{\boldsymbol{\theta}^{\star}}+\operatorname{tr}\left[\left(\mathbf{I}-\mathbf{M}^{\star}\right)(\mathbf{I}-\mathbf{Q}) V(\mathbf{e})(\mathbf{I}-\mathbf{Q})^{T}\right)\right] .
$$

\subsubsection{Proof of (5.18)}

By definition of the conditional variance, we have

$$
V\left(M S E \mid \boldsymbol{\theta}^{\star}\right)=E\left[\left\{M S E-E\left(M S E \mid \boldsymbol{\theta}^{\star}\right)\right\}^{2} \mid \boldsymbol{\theta}^{\star}\right] .
$$

Let $\phi_{1}=\sum_{i=1}^{m} \sum_{j, k=1}^{n_{i}}\left(\delta_{i j k}-h_{i j k}^{\star}\right) c_{i j k}^{\star}$, then using (5.16), we have

$$
M S E-E\left(M S E \mid \boldsymbol{\theta}^{\star}\right)=\frac{\mathbf{y}^{\star T}\left(\mathbf{I}-\mathbf{P}^{\star}\right) \mathbf{y}^{\star}}{n-p}-\frac{\boldsymbol{\theta}^{\star T}\left(\mathbf{I}-\mathbf{P}^{\star}\right) \boldsymbol{\theta}^{\star}}{n-p}-\frac{1}{n-p} \phi_{1} .
$$

Substituting $\mathbf{y}^{\star}$ with $\boldsymbol{\theta}^{\star}+\mathbf{e}^{\star}$, we get:

$$
M S E-E\left(M S E \mid \boldsymbol{\theta}^{\star}\right)=\frac{1}{n-p}\left\{2 \boldsymbol{\theta}^{\star T}\left(\mathbf{I}-\mathbf{P}^{\star}\right) \mathbf{e}^{\star}+\mathbf{e}^{\star T}\left(\mathbf{I}-\mathbf{P}^{\star}\right) \mathbf{e}^{\star}-\phi_{1}\right\} .
$$

Hence,

$$
E\left[\left\{M S E-E\left(M S E \mid \boldsymbol{\theta}^{\star}\right)\right\}^{2} \mid \boldsymbol{\theta}^{\star}\right]=\left(\frac{1}{n-p}\right)^{2} E\left[\left\{2 \boldsymbol{\theta}^{\star T}\left(\mathbf{I}-\mathbf{P}^{\star}\right) \mathbf{e}^{\star}+\mathbf{e}^{\star T}\left(\mathbf{I}-\mathbf{P}^{\star}\right) \mathbf{e}^{\star}-\phi_{1}\right\}^{2}\right]
$$


After some algebra, (5.35) can be written as

$$
E\left[\left\{M S E-E\left(M S E \mid \boldsymbol{\theta}^{\star}\right)\right\}^{2} \mid \boldsymbol{\theta}^{\star}\right]=\left(\frac{1}{n-p}\right)^{2}\{A+B+C\}
$$

where

$$
\begin{aligned}
& A=E\left[\left\{\left(\mathbf{e}^{\star T}\left(\mathbf{I}-\mathbf{P}^{\star}\right) \mathbf{e}^{\star}\right)\right\}^{2}\right]-\phi_{1}^{2} \\
& B=4 \boldsymbol{\theta}^{\star T}\left(\mathbf{I}-\mathbf{P}^{\star}\right) E\left[\mathbf{e}^{\star} \mathbf{e}^{\star T}\left(\mathbf{I}-\mathbf{P}^{\star}\right) \mathbf{e}^{\star}\right] \\
& C=4 E\left[\left\{\boldsymbol{\theta}^{\star}\left(\mathbf{I}-\mathbf{P}^{\star}\right) \mathbf{e}^{\star}\right\}^{2}\right] .
\end{aligned}
$$

We expand $A, B$ and $C$ in (5.36) separately.

The term $A$ can be simplified as follows

$$
A=V\left[\left(\mathbf{e}^{\star T}\left(\mathbf{I}-\mathbf{P}^{\star}\right) \mathbf{e}^{\star}\right)\right]+E^{2}\left[\left(\mathbf{e}^{\star T}\left(\mathbf{I}-\mathbf{P}^{\star}\right) \mathbf{e}^{\star}\right)\right]-\phi_{1}^{2} .
$$

It was shown in subsection 5.6.1 that $E\left[\mathbf{e}^{\star T}\left(\mathbf{I}-\mathbf{P}^{\star}\right) \mathbf{e}^{\star}\right]=\phi_{1}$, so the above equation can be further simplified to

$$
\begin{aligned}
A & =\left\{V\left[\left(\mathbf{e}^{\star T}\left(\mathbf{I}-\mathbf{P}^{\star}\right) \mathbf{e}^{\star}\right)\right]+\phi_{1}^{2}-\phi_{1}^{2}\right\} \\
& =V\left[\left(\mathbf{e}^{\star T}\left(\mathbf{I}-\mathbf{P}^{\star}\right) \mathbf{e}^{\star}\right)\right] .
\end{aligned}
$$

In a basic two-fold subarea model, it is assumed that $\mathbf{e} \sim \operatorname{Normal}(\mathbf{0}, \boldsymbol{\Sigma})$, where $\boldsymbol{\Sigma}$ is a $n \times n$ diagonal matrix with diagonal elements equal to $\left(\psi_{11}, \psi_{12}, \cdots\right.$, $\left.\psi_{1 n_{1}}, \psi_{21}, \cdots, \psi_{2 n_{2}}, \cdots, \psi_{m 1}, \cdots, \psi_{m n_{m}}\right)$. As shown in subsection 5.6.1, $\mathbf{e}^{\star}=(\mathbf{I}-\mathbf{Q}) \mathbf{e}$ as a result, $\mathbf{e}^{\star} \sim N\left(\mathbf{0}, \boldsymbol{\Sigma}^{\star}\right)$, where $\boldsymbol{\Sigma}^{\star}=(\mathbf{I}-\mathbf{Q}) \mathbf{\Sigma}(\mathbf{I}-\mathbf{Q})$.

Using normality features of $\mathbf{e}^{\star}$ we have:

$V\left[\left(\mathbf{e}^{\star T}\left(\mathbf{I}-\mathbf{P}^{\star}\right) \mathbf{e}^{\star}\right)\right]=\operatorname{Tr}\left(2\left(\mathbf{I}-\mathbf{P}^{\star}\right) \boldsymbol{\Sigma}^{\star}\left(\mathbf{I}-\mathbf{P}^{\star}\right) \mathbf{\Sigma}^{\star}\right)$ (see page 43 of Petersen and 
Pedersen (2012)).

Therefore, (5.37) can be simplified to:

$$
\begin{aligned}
A & =\operatorname{Tr}\left(2\left(\mathbf{I}-\mathbf{P}^{\star}\right) \boldsymbol{\Sigma}^{\star}\left(\mathbf{I}-\mathbf{P}^{\star}\right) \mathbf{\Sigma}^{\star}\right) \\
& =2 \operatorname{Tr}\left(\left(\mathbf{I}-\mathbf{P}^{\star}\right) \boldsymbol{\Sigma}^{\star}\left(\mathbf{I}-\mathbf{P}^{\star}\right) \boldsymbol{\Sigma}^{\star}\right) .
\end{aligned}
$$

The trace of the square forms of $\left(\mathbf{I}-\mathbf{P}^{\star}\right) \boldsymbol{\Sigma}^{\star}$ in the above equation can be expanded using the following theorem.

Theorem 2 For any two real matrices $\mathbf{A}$ and $\mathbf{B}$ of the same order, we have $\operatorname{Tr}\left(\mathbf{A}^{T} \mathbf{B}\right)^{2} \leq \operatorname{Tr}\left(\mathbf{A}^{T} \mathbf{A}\right)\left(\mathbf{B}^{T} \mathbf{B}\right)$ with equality if and and only if $\mathbf{A B}^{T}=\mathbf{B} \mathbf{A}^{T}$.

Proof of theorem 2 is given in page 201 of Magnus and Neudecker (1988).

Thus, equation (5.38) can be written as

$$
A \leq 2 \operatorname{Tr}\left(\left(\mathbf{I}-\mathbf{P}^{\star}\right)\left(\mathbf{I}-\mathbf{P}^{\star}\right) \mathbf{\Sigma}^{\star} \mathbf{\Sigma}^{\star}\right)
$$

Since $\left(\mathbf{I}-\mathbf{P}^{\star}\right)$ is idempotent and $\mathbf{P}^{\star}$ (i.e., Hat matrix) is positive definite, we have

$$
\begin{aligned}
A & \leq 2 \operatorname{Tr}\left(\left(\mathbf{I}-\mathbf{P}^{\star}\right) \mathbf{\Sigma}^{\star 2}\right) \\
& \leq 2 \operatorname{Tr}\left(\boldsymbol{\Sigma}^{\star 2}\right) .
\end{aligned}
$$

In order to find $\operatorname{Tr}\left(\Sigma^{\star 2}\right)$, we first expand $\Sigma^{\star 2}$. It should be noted that $\Sigma^{\star}$ is the same as

$$
\Sigma^{\star}=\operatorname{diag}\left(V_{i}^{\star}\right)_{1 \leq i \leq m},
$$

Hence,

$$
\Sigma^{\star 2}=\operatorname{diag}\left(V_{i}^{\star 2}\right)_{1 \leq i \leq m}
$$


where

$$
\begin{aligned}
V_{i}^{\star 2} & =\operatorname{var}\left(\mathbf{e}_{i}^{\star}\right) \operatorname{var}\left(\mathbf{e}_{i}^{\star}\right) \\
& =\left[\begin{array}{cccc}
v\left(e_{i 1}^{\star}\right) & \operatorname{cov}\left(e_{i 1}^{\star}, e_{i 2}^{\star}\right) & \ldots & \operatorname{cov}\left(e_{i 1}^{\star}, e_{i n_{i}}^{\star}\right) \\
\operatorname{cov}\left(e_{i 2}^{\star}, e_{i 1}^{\star}\right) & v\left(e_{i 2}^{\star}\right) & \ldots & \operatorname{cov}\left(e_{i 2}^{\star}, e_{i n_{i}}^{\star}\right) \\
\vdots & \vdots & \ddots & \vdots \\
\operatorname{cov}\left(e_{i n_{i}}^{\star}, e_{i 1}^{\star}\right) & \ldots & \ldots & v\left(e_{i n_{i}}^{\star}\right)
\end{array}\right]\left[\begin{array}{cccc}
v\left(e_{i 1}^{\star}\right) & \operatorname{cov}\left(e_{i 1}^{\star}, e_{i 2}^{\star}\right) & \ldots & \operatorname{cov}\left(e_{i 1}^{\star}, e_{i n_{i}}^{\star}\right) \\
\operatorname{cov}\left(e_{i 2}^{\star}, e_{i 1}^{\star}\right) & v\left(e_{i 2}^{\star}\right) & \ldots & \operatorname{cov}\left(e_{i 2}^{\star}, e_{i n_{i}}^{\star}\right) \\
\vdots & \vdots & \ddots & \vdots \\
\operatorname{cov}\left(e_{i n_{i}}^{\star}, e_{i 1}^{\star}\right) & \ldots & \ldots & v\left(e_{i n_{i}}^{\star}\right)
\end{array}\right] .
\end{aligned}
$$

After multiplying the above matrices, the diagonal elements of $V_{i}^{\star 2}$, which are needed for the trace, are

$$
\begin{aligned}
& v\left(e_{i 1}^{\star}\right)^{2}+\operatorname{cov}\left(e_{i 1}^{\star}, e_{i 2}^{\star}\right)^{2}+\cdots+\operatorname{cov}\left(e_{i 1}^{\star}, e_{i n_{i}}^{\star}\right)^{2}, \\
& \operatorname{cov}\left(e_{i 2}^{\star}, e_{i 2}^{\star}\right)^{2}+v\left(e_{i 2}^{\star}\right)^{2}+\cdots+\operatorname{cov}\left(e_{i 2}^{\star}, e_{i n_{i}}^{\star}\right)^{2}, \\
& \vdots \\
& \operatorname{cov}\left(e_{i n_{i}}^{\star}, e_{i 1}^{\star}\right)^{2}+\operatorname{cov}\left(e_{i n_{i}}^{\star}, e_{i 2}^{\star}\right)^{2}+\cdots+v\left(e_{i n_{i}}^{\star}\right)^{2} .
\end{aligned}
$$

Hence, (5.39) can be written as:

$$
\begin{aligned}
A & \leq 2 \operatorname{Tr}\left(\boldsymbol{\Sigma}^{\star 2}\right) \\
& =2 \sum_{i=1}^{m} \sum_{j, k=1}^{n_{i}}\left\{v\left(e_{i j}^{\star}\right)\right\}^{2}+\left\{\operatorname{cov}\left(e_{i j}^{\star}, e_{i k}^{\star}\right)\right\}^{2}
\end{aligned}
$$

It is assumed that $\psi_{i j}<\psi_{0}<\infty$, which means $E\left(e_{i j}^{2}\right)$ is uniformly bounded. As a result, $v\left(e_{i j}^{\star}\right)$ is also uniformly bounded by some $\gamma>0$, for all $i$ and $j$. Moreover,

$$
\left|\operatorname{cov}\left(e_{i j}^{\star}, e_{i k}^{\star}\right)\right| \leq \sqrt{v\left(e_{i j}^{\star}\right) v\left(e_{i k}^{\star}\right)} \leq \gamma
$$

Hence, we have

$$
\left\{v\left(e_{i j}^{\star}\right)\right\}^{2}+\left\{\operatorname{cov}\left(e_{i j}^{\star}, e_{i k}^{\star}\right)\right\}^{2} \leq 2 \gamma^{2} .
$$


Going back to (5.40), we have

$$
\begin{aligned}
A & \leq 2 \sum_{i=1}^{m} n_{i}\left(2 \gamma^{2}\right) \\
& =4 n \gamma^{2}
\end{aligned}
$$

We now expand $B=4 \boldsymbol{\theta}^{\star T}\left(\mathbf{I}-\mathbf{P}^{\star}\right) E\left[\mathbf{e}^{\star} \mathbf{e}^{\star T}\left(\mathbf{I}-\mathbf{P}^{\star}\right) \mathbf{e}^{\star}\right]$ in (5.36). Using the normality features of $\mathbf{e}^{\star}, E\left[\mathbf{e}^{\star} \mathbf{e}^{\star T}\left(\mathbf{I}-\mathbf{P}^{\star}\right) \mathbf{e}^{\star}\right]=0$, as $E\left(\mathbf{e}^{\star}\right)=0$. As a result $B=0$.

For term $C$ in (5.36) we have:

$$
\begin{aligned}
C & =4 E\left[\left\{\boldsymbol{\theta}^{\star}\left(\mathbf{I}-\mathbf{P}^{\star}\right) \mathbf{e}^{\star}\right\}^{2}\right] \\
& \left.=4 E\left[\boldsymbol{\theta}^{\star}\left(\mathbf{I}-\mathbf{P}^{\star}\right) \mathbf{e}^{\star} \mathbf{e}^{\star T} \mathbf{I}-\mathbf{P}^{\star}\right) \boldsymbol{\theta}^{\star T}\right] \\
& =4 \boldsymbol{\theta}^{\star T}\left(\mathbf{I}-\mathbf{P}^{\star}\right) \boldsymbol{\Sigma}^{\star}\left(\mathbf{I}-\mathbf{P}^{\star}\right) \boldsymbol{\theta}^{\star}
\end{aligned}
$$

Since (5.41) is a number, it is equal to its own trace:

$$
C=4 \operatorname{Tr}\left(\boldsymbol{\theta}^{\star T}\left(\mathbf{I}-\mathbf{P}^{\star}\right) \boldsymbol{\Sigma}^{\star}\left(\mathbf{I}-\mathbf{P}^{\star}\right) \boldsymbol{\theta}^{\star}\right)
$$

Using the cyclic property of trace and Theorem 2, we have

$$
\begin{aligned}
C & \leq 4 \sqrt{\operatorname{Tr}\left(\Sigma^{\star^{2}}\right) \operatorname{Tr}\left(\mathbf{U}^{T} \mathbf{U}\right)} \\
& \leq 4 \sqrt{\operatorname{Tr}\left(\Sigma^{\star^{2}}\right)}
\end{aligned}
$$

where $\mathbf{U}=\left(\mathbf{I}-\mathbf{P}^{\star}\right) \boldsymbol{\theta}^{\star} \boldsymbol{\theta}^{\star T}\left(\mathbf{I}-\mathbf{P}^{\star}\right)$. We have already showed that $\operatorname{Tr}\left(\boldsymbol{\Sigma}^{\star 2}\right) \leq 2 n \gamma^{2}$, so $C \leq 4 \sqrt{2 n} \gamma$. 
Going back to (5.36), we have

$$
\begin{aligned}
E\left[\left(M S E-E\left(M S E \mid \boldsymbol{\theta}^{\star}\right)^{2} \mid \boldsymbol{\theta}^{\star}\right]\right. & \leq\left(\frac{1}{n-p}\right)^{2}\left\{4 n \gamma^{2}+0+4 \sqrt{2 n} \gamma\right\} \\
& \leq \frac{q}{n} \quad(q>0),
\end{aligned}
$$

which means $V\left(M S E \mid \boldsymbol{\theta}^{\star}\right)=O\left(\frac{1}{n}\right)$ (or $O\left(\frac{1}{m}\right)$ as we assumed that the number of subareas, $n_{i}$, is bounded and $m \rightarrow \infty$.

The proof for $V\left(M S T \mid \boldsymbol{\theta}^{\star}\right)$ is similar, where we replace $\phi_{1}$ with $\phi_{2}=$ and $\mathbf{P}^{\star}$ with $\mathbf{M}^{\star}$.

\subsubsection{Proof of (5.19)}

By $o_{p}$ definition we have: $X_{n}-X=o_{p}(1)$ iff $X_{n} \stackrel{p}{\rightarrow} X$. So, we should show that $M S E \stackrel{p}{\rightarrow} E\left(M S E \mid \boldsymbol{\theta}^{\star}\right)$ which means: $\lim _{n \rightarrow \infty} P\left(\left|M S E-E\left(M S E \mid \boldsymbol{\theta}^{\star}\right)\right| \geq \epsilon\right)=0$ for all $\epsilon>0$.

By Chebyshev's inequality we have:

$$
\left.P\left(\left[M S E-E\left(M S E \mid \boldsymbol{\theta}^{\star}\right)\right]^{2}\right) \geq \epsilon^{2}\right) \leq \frac{E\left(\left[M S E-E\left(M S E \mid \boldsymbol{\theta}^{\star}\right)\right]^{2}\right)}{\epsilon^{2}}
$$

So, we need to show that $E\left(\left[M S E-E\left(M S E \mid \boldsymbol{\theta}^{\star}\right)\right]^{2}\right)$ goes to zero as $n \rightarrow \infty$.

By (5.18), we have $\operatorname{Var}\left(M S E \mid \boldsymbol{\theta}^{\star}\right) \leq \frac{M}{n}$ for all $n>n_{0}$. So, we have: $\lim _{n \rightarrow \infty} E\left(\left[M S E-E\left(M S E \mid \boldsymbol{\theta}^{\star}\right)\right]^{2}\right) \leq \lim _{n \rightarrow \infty} \frac{M}{n}=0$, which means $M S E \stackrel{p}{\rightarrow} E\left(M S E \mid \boldsymbol{\theta}^{\star}\right)$. Similarly, for MST we have

$$
\left.P\left(\left[M S T-E\left(M S T \mid \boldsymbol{\theta}^{\star}\right)\right]^{2}\right) \geq \epsilon^{2}\right) \leq \frac{E\left(\left[M S T-E\left(M S T \mid \boldsymbol{\theta}^{\star}\right)\right]^{2}\right)}{\epsilon^{2}}
$$


By (5.18), we have $\operatorname{Var}\left(M S T \mid \boldsymbol{\theta}^{\star}\right) \leq \frac{M}{n}$ for all $n>n_{0}$.

Hence, $\lim _{n \rightarrow \infty} E\left(\left[M S T-E\left(M S T \mid \boldsymbol{\theta}^{\star}\right)\right]^{2}\right) \leq \lim _{n \rightarrow \infty} \frac{M}{n}=0$, which means $M S T \stackrel{p}{\rightarrow} E\left(M S T \mid \boldsymbol{\theta}^{\star}\right)$.

\subsection{Appendix}

Probabilities of model selection using $R^{2}$ (adj)

Table 5.4: Probabilities of model selection using $R^{2}(a d j)_{\text {naive } 1}$

\begin{tabular}{||ccccccc||}
\hline \hline \multicolumn{7}{|c||}{$\beta=(2,0,0,4,8)^{T}$} \\
\hline Model & Category & $\sigma_{v}^{2}=1$ & $\sigma_{v}^{2}=5$ & $\sigma_{v}^{2}=10$ & $\sigma_{v}^{2}=16$ & $\sigma_{v}^{2}=24$ \\
\hline$(0,3,4)$ & optimal & 49.1 & 43.9 & 37.0 & 33.7 & 31.2 \\
$(0,1,3,4)$ & I & 19.3 & 19.1 & 18.9 & 15.5 & 12.8 \\
$(0,2,3,4)$ & I & 21.0 & 22.2 & 21.0 & 18.6 & 18.3 \\
$(0,1,3,4)$ & I & 10.6 & 9.2 & 9.5 & 8.9 & 7.1 \\
$(0,3)$ & II & 0.0 & 3.4 & 5.7 & 11.3 & 15.6 \\
$(0,1,3)$ & II & 0.0 & 1.6 & 3.1 & 4.9 & 5.4 \\
$(0,2,3)$ & II & 0.0 & 0.8 & 3.5 & 5.6 & 6.8 \\
$(0,1,2,3)$ & II & 0.0 & 0.4 & 1.3 & 1.5 & 2.8 \\
\hline \hline & & & $\beta=(2,3,0,4,8)^{T}$ & & & \\
\hline Model & Category & $\sigma_{v}^{2}=1$ & $\sigma_{v}^{2}=5$ & $\sigma_{v}^{2}=10$ & $\sigma_{v}^{2}=16$ & $\sigma_{v}^{2}=24$ \\
\hline$(0,1,3,4)$ & optimal & 65.3 & 59.5 & 54.4 & 49.0 & 47.2 \\
$(0,1,2,3,4)$ & I & 34.7 & 34.9 & 30.3 & 26.3 & 21.6 \\
$(0,1,3)$ & II & 0.0 & 4.1 & 11.8 & 16.2 & 19.7 \\
$(0,1,2,3)$ & II & 0.0 & 1.5 & 3.5 & 8.5 & 11.5 \\
\hline \hline Model & Category & $\sigma_{v}^{2}=1$ & $\sigma_{v}^{2}=5$ & $\sigma_{v}^{2}=10$ & $\sigma_{v}^{2}=16$ & $\sigma_{v}^{2}=24$ \\
\hline$(0,1,2,3,4)$ & optimal & 100 & 96.3 & 85.4 & 76 & 67.1 \\
$(0,1,2,3)$ & II & 0.0 & 3.7 & 14.6 & 24 & 32.9 \\
\hline \hline
\end{tabular}


Table 5.5: Probabilities of model selection using $R^{2}(a d j)_{\text {naive2 }}$

\begin{tabular}{||ccccccc||}
\hline \hline \multicolumn{7}{c||}{$\beta=(2,0,0,4,8)^{T}$} \\
Model & Category & $\sigma_{v}^{2}=1$ & $\sigma_{v}^{2}=5$ & $\sigma_{v}^{2}=10$ & $\sigma_{v}^{2}=16$ & $\sigma_{v}^{2}=24$ \\
\hline$(0,3,4)$ & optimal & 47.6 & 43.9 & 37.2 & 33.6 & 31.2 \\
$(0,1,3,4)$ & I & 20.5 & 19.1 & 18.8 & 15.5 & 12.9 \\
$(0,2,3,4)$ & I & 21.9 & 22.2 & 21.3 & 18.6 & 18.0 \\
$(0,1,2,3,4)$ & I & 10.0 & 9.2 & 9.2 & 8.9 & 7.4 \\
$(0,1,2,3)$ & II & 0.0 & 0.4 & 1.4 & 1.6 & 2.8 \\
$(0,3)$ & II & 0.0 & 3.4 & 5.9 & 11.3 & 15.5 \\
$(0,1,3)$ & II & 0.0 & 1.0 & 2.8 & 4.9 & 5.4 \\
$(0,2,3)$ & II & 0.0 & 0.8 & 3.4 & 5.6 & 6.8 \\
\hline \hline & & & $\beta=(2,3,0,4,8)^{T}$ & & & \\
\hline Model & Category & $\sigma_{v}^{2}=1$ & $\sigma_{v}^{2}=5$ & $\sigma_{v}^{2}=10$ & $\sigma_{v}^{2}=16$ & $\sigma_{v}^{2}=24$ \\
\hline$(0,1,3,4)$ & optimal & 65.1 & 60.0 & 54.6 & 49.0 & 47.2 \\
$(0,1,2,3,4)$ & II & 34.9 & 34.5 & 30.2 & 26.3 & 21.6 \\
$(0,1,2,3)$ & II & 0.0 & 1.6 & 3.5 & 8.5 & 11.6 \\
$(0,1,3)$ & II & 0.0 & 3.9 & 11.7 & 16.2 & 19.6 \\
\hline \hline Model & Category & $\sigma_{v}^{2}=1$ & $\sigma_{v}^{2}=5$ & $\sigma_{v}^{2}=10$ & $\sigma_{v}^{2}=16$ & $\sigma_{v}^{2}=24$ \\
\hline$(0,1,2,3,4)$ & optimal & 100 & 96.2 & 85.4 & 75.9 & 67 \\
$(0,1,2,3)$ & II & 0.0 & 3.8 & 14.6 & 24.1 & 33.0 \\
\hline \hline
\end{tabular}


Table 5.6: Probabilities of model selection using $R^{2}(a d j)_{n e w}$

\begin{tabular}{|c|c|c|c|c|c|c|}
\hline \multicolumn{7}{|c|}{$\beta=(2,0,0,4,8)^{T}$} \\
\hline Model & Category & $\sigma_{v}^{2}=1$ & $\sigma_{v}^{2}=5$ & $\sigma_{v}^{2}=10$ & $\sigma_{v}^{2}=16$ & $\sigma_{v}^{2}=24$ \\
\hline$(0,3,4)$ & optimal & 92.1 & 90.5 & 93.2 & 91.1 & 91.5 \\
\hline$(0,1,3,4)$ & I & 3.2 & 3.9 & 3.5 & 3.5 & 4.1 \\
\hline$(0,2,3,4)$ & I & 3.4 & 4.4 & 2.8 & 4.4 & 3.9 \\
\hline$(0,1,2,3,4)$ & I & 0.6 & 0.3 & 0.2 & 0.3 & 0.3 \\
\hline$(0,3)$ & II & 0.7 & 0.9 & 0.3 & 0.7 & 0.2 \\
\hline \multicolumn{7}{|c|}{$\beta=(2,3,0,4,8)^{T}$} \\
\hline Model & Category & $\sigma_{v}^{2}=1$ & $\sigma_{v}^{2}=5$ & $\sigma_{v}^{2}=10$ & $\sigma_{v}^{2}=16$ & $\sigma_{v}^{2}=24$ \\
\hline$(0,1,3,4)$ & optimal & 94.6 & 95.2 & 94.0 & 94.8 & 94.6 \\
\hline$(0,1,2,3,4)$ & I & 5.1 & 4.4 & 5.0 & 4.5 & 4.1 \\
\hline$(0,1,3)$ & II & 0.3 & 0.4 & 0.9 & 0.7 & 1.3 \\
\hline$(0,1,2,3)$ & II & 0.0 & 0.0 & 0.1 & 0.0 & 0.0 \\
\hline \multicolumn{7}{|c|}{$\beta=(2,3,5,4,8)^{T}$} \\
\hline Model & Category & $\sigma_{v}^{2}=1$ & $\sigma_{v}^{2}=5$ & $\sigma_{v}^{2}=10$ & $\sigma_{v}^{2}=16$ & $\sigma_{v}^{2}=24$ \\
\hline$(0,1,2,3,4)$ & optimal & 99.9 & 99.1 & 99.8 & 99.6 & 99.2 \\
\hline$(0,1,2,3)$ & II & 0.1 & 0.9 & 0.2 & 0.4 & 0.8 \\
\hline
\end{tabular}




\section{Chapter 6}

\section{Future Research}

In this thesis, we focused on three problems in small area estimation: 1) small area estimation under informative sampling, 2) EB estimation under unmatched twofold subarea models and 3) variable selection under a two-fold subarea model. For

the first problem, we proposed a semi-parametric approach by including a P-spline term of selection probabilities in the model to account for the informativeness of the sampling design. In addressing the second problem, we proposed an empirical best approach to the estimation of small subarea means. For the third problem, we developed a two-step adjustment to standard variable selection methods, by integrating the methods for the basic area level and the basic unit level model. Some future research ideas are discussed below, for improving some aspects of the proposed methods.

In Chapter 2, the simulation study showed that the proposed P-spline augmentation method performs well in terms of bias and MSE, and has some advantages over other methods in the literature, as it can be used for any design and does not need to assume a specific model for survey weights. We focused on 
the EBLUP estimator of small area means under the proposed P-spline model. Alternatively, the pseudo-EBLUP estimator of $\mu_{i}$ can be derived. Verret et al. (2015) obtained the pseudo-EBLUP under a parametric augmented model and compared its performance with the corresponding EBLUP. They noted that the pseudo-EBLUP provides better results. Moreover, Hidiroglou and You (2016) compared the EBLUP and pseudo-EBLUP estimators for small area estimation under the nested error regression model and pointed out that the pseudo-EBLUP estimator has the best performance in terms of bias and coverage rate under informative sampling. Considering the positive outcome of those studies, it would be interesting to study the pseudo-EBLUP under the P-spline model.

In Chapter 3, we studied the case where not all areas are sampled and extended the P-spline method to two-stage informative sampling. For non-sampled areas, some assumptions about the availability of selection probabilities need to be made. A Monte Carlo simulation study was conducted under two scenarios, and the performance of the P-spline method was compared with methods proposed by Pfeffermann and Sverchkov (2007) and Verret et al. (2015). The method by Pfeffermann and Sverchkov provided better results in terms of bias. However, it should be noted that their method assumes the knowledge of models for the sampling weights, which has limited use in practice. For estimating the MSE, similar to the one-stage sampling case, a conditional bootstrap method was proposed. The drawback of the proposed bootstrap method for a two-stage sampling is that is that $p_{i}$ and $p_{j \mid i}$ values should be known for all units in the population, which may not be feasible in practice. Therefore, to improve the method, we should investigate alternative methods that do not require the knowledge of selection probabilities in non-sampled areas. 
The two-fold subarea model under unmatched sampling and linking model was studied in Chapter 4. EBUP estimators of subarea means were obtained. A parametric bootstrap method was proposed for estimating the MSE of the EBUP estimators. The main advantage of the parametric bootstrap method is its simplicity to implement. However, it may produce biased inference if the underlying distributions are misspecified. We may investigate MSE estimation for EBUP using Prasad and Rao (1990) decomposition. We extended the proposed method to a three-fold model under unmatched linking and sampling models. The evaluation of the three-fold model as well as the estimation of MSE have remained for future work.

In Chapter 5, we focused on matched two-fold subarea level models and proposed a two-step adjustment to the standard variable selection. We used the Fuller-Battese transformation which needs information on $\rho$. Li and Lahiri (2018) used a different transformation suggested in Lahiri and Li (2009) (called the "LL" transformation). The LL transformation does not depend on $\rho$. In Section 5.3.3 the process of estimating $\rho$ using the data was presented, which is an iterative compute-intensive process. Li and Lahiri (2018) noted that LL transformation provides similar results as Fuller-Battese transformation. Using the LL transformation in the proposed two-step adjustment for a two-fold model will be studied in the future. Moreover, the vairaoble selction methods under unmatched two-fold model has remained for future work. 


\section{Bibliography}

Akaike, H. (1973). Maximum Likelihood Identification of Gaussian Autoregressive Moving Average Models. Biometrika, 60(1):255-265.

Battese, G. E., Harter, R. M., and Fuller, W. A. (1988). An Error Components Model for Prediction of County Crop Areas Using Survey and Satellite Data. Journal of the American Statistical Association, 83(401):28-36.

Brackstone, G. J. (1987). Small Area Data: Policy Issues and Technical Challenges, pages 3-20. John Wiley \& Sons.

Carlin, B. P. and Louis, T. A. (2008). Bayesian Methods for Data Analysis. Chapman and Hall/CRC.

Chatrchi, G. (2012). Robust estimation of variance components in small area estimation. Master's thesis, Carleton University, Ottawa, Canada.

Chatterjee, S. and Lahiri, P. (2007). A Simple Computational Method for Estimating Mean Squared Prediction Error in General Small Area Model. In Association, A. S., editor, In Proceedings of the section on survey research methods, pages 3486-3493.

Cochran, W. G. (2007). Sampling Techniques. John Wiley \& Sons.

Dempster, A. P., Laird, N. M., and Rubin, D. B. (1977). Maximum Likelihood from Incomplete Data via the EM Algorithm. Journal of the Royal Statistical Society, Series B (methodological):1-38.

Fay, R. E. and Herriot, R. A. (1979). Estimates of Income for Small Places: An Application of James-Stein Procedures to Census Data. Journal of the American Statistical Association, 74(366a):269-277.

Florescu, I. (2014). Probability and Stochastic Processes. John Wiley \& Sons.

Fuller, W. A. and Battese, G. E. (1973). Transformations for Estimation of Linear Models With Nested-Error Structure. Journal of the American Statistical association, 68(343):626-632. 
Gonzalez, M. E. (1973). Use and evaluation of synthetic estimates. In Proceedings of the social statistics section, American Statistical Association, 1973., pages $33-36$.

Hall, P. and Maiti, T. (2006). On Parametric Bootstrap Methods for Small Area Prediction. Journal of the Royal Statistical Society: Series B (Statistical Methodology), 68(2):221-238.

Henderson, C. R. (1953). Estimation of Variance and Covariance Components. Biometrics, 9(2):226-252.

Henderson, C. R. (1963). Selection Index and Expected Genetic Advance. Statistical Genetics and Plant Breeding, 982:141-163.

Henderson, C. R. (1975). Best Linear Unbiased Estimation and Prediction Under a Selection Model. Biometrics, (423-447).

Hidiroglou, M. A. and You, Y. (2016). Comparison of Unit Level and Area Level Small Area Estimators. Survey Methodology, 42(41-61).

Hocking, R. R. and Kutner, M. H. (1975). Some analytical and numerical comparisons of estimators for the mixed AOV model. Biometrics, pages 19-28.

Ibrahim, J. G., Zhu, H., Garcia, R. I., and Guo, R. (2011). Fixed and Random Effects Selection in Mixed Effects Models. Biometrics, 67(2):495-503.

James, W. and Stein, C. (1961). Estimation with Quadratic Loss. In Proceedings of the Fourth Berkeley Symposium on Mathematical Statistics and Probability, volume 1, pages 361-379.

Jiang, J. and Lahiri, P. (2006). Mixed Model Prediction and Small Area Estimation. Test, 15(1):1-96.

Jiang, J., Lahiri, P., and Wan, S. M. (2002). A Unified jackknife Theory for Empirical Best Prediction With M-estimation. The Annals of Statistics, 30(6):17821810 .

Kim, J. and Skinner, C. (2013). Weighting in survey analysis under informative sampling. Biometrika, 100, 385-398.:385-398.

Lahiri, P. and Li, Y. (2009). A New Alternative to the Standard F Test for Clustered Data. Journal of Statistical Planning and Inference, 139(10):34303441. 
Lahiri, P. and Suntornchost, J. (2015). Variable Selection for Linear Mixed Models With Applications in Small Area Estimation. Sankhya: The Indian Journal of Statistics, 77-B(Part 2):312-320.

Li, Y. and Lahiri, P. (2018). A Simple Adaptation of Variable Selection Software for Regression Models to Select Variables in Nested Error Regression Models. Sankhya B, pages 1-16.

Liang, H., Wu, H., and Zou, G. (2008). A Note on Conditional AIC for Linear Mixed-effects Models. Biometrika,, 95(3):773-778.

Lin, X. (1997). Variance Component Testing in Generalised Linear Models With Random Effects. Biometrika, 84:309-326.

Lohr, S. (2009). Sampling: design and analysis. Nelson Education.

Magnus, J. R. and Neudecker, H. (1988). Matrix Differential Calculus With Applications in Statistics and Econometrics. Wiley series in Probability and Mathematical Statistics.

Mallows, C. (1973). Some Comments on CP. Technometrics,, 15(4):661-675.

Manitz, J. (2012). Samplingbook: Survey Sampling Procedures. URL, http://CRAN. R-project. org/package= samplingbook, (contributions by M. Hempelmann, G. Kauermann, H. Kuechenhoff, S. Shao, C. Oberhauser, N. Westerheide, M. Wiesenfarth).

Maritz, J. S. and Lwin, T. (1989). Empirical Bayes Methods. Chapman and Hall/CRC., second edition edition.

McCulloch, C. E. and Searle, S. R. (2004). Generalized, Linear, and Mixed models. John Wiley \& Sons.

Meza, J. and Lahiri, P. (2005). A Note on the Cp Statistic Under the Nested Error Regression Model. Survey Methodology, 31(105-109).

Mohadjer, L., Rao, J. N. K., Liu, B., Krenzke, T., and de Kerckhove, W. V. (2012). Hierarchical Bayes Small Area Estimates of Adult Literacy Using Unmatched Sampling and Linking Models. Journal of the Indian Agricultural Statistics, 66:55-63.

Morris, C. N. (1983). Parametric Empirical Bayes Inference: Theory and Applications. Journal of the American Statistical Association, 78(381):47-55. 
Opsomer, J. D., Claeskens, G., Ranalli, M. G., Kauermann, G., and Breidt, F. J. (2008). Nonparametric Small Area Estimation Using Penalized Spline Regression. Journal of the Royal Statistical Society: Series B (Statistical Methodology), $70(1): 265-286$.

Petersen, K. B. and Pedersen, M. S. (2012). The Matrix Cookbook. Technical University of Denmark.

Pfeffermann, D. and Glickman, H. (2004). Mean Square Error Approximation in Small Area Estimation by Use of Parametric and Nonparametric Bootstrap. ASA Section on Survey Research Methods, (4167-4178).

Pfeffermann, D. and Sverchkov, M. (2007). Small Area Estimation Under Informative Probability Sampling of Areas and Within the Selected Areas. Journal of the American Statistical Association, 102(480):427-439.

Prasad, N. G. N. and Rao, J. N. K. (1990). The Estimation of the Mean Squared Error of Small Area Estimators. Journal of the American Statistical Association, 85(409):163-171.

Rao, J. N. K. (1965). On Two Simple Schemes of Unequal Probability Sampling Without Replacement. Journal of the Indian Statistical Association, 3:173-180.

Rao, J. N. K. and Molina, I. (2015). Small Area Estimation. John Wiley \& Sons, 2nd edition.

Rao, J. N. K., Sinha, S. K., and Dumitrescu, L. (2014). Robust Small Area Estimation Under Semiparametric Mixed Models. Canadian Journal of Statistics, 42(1):126-141.

Rao, J. N. K., Sutradhar, B. C., and Yue, K. (1993). Generalized Least Squares F Test in Regression Analysis With Two-stage Cluster Samples. Journal of the American Statistical Association, 88(424):1388-1391.

Robert, C. and Casella, G. (2004). Monte Carlo Statistical Methods. Springer, New York, NY.

Rodriguez, G. (2011). Smoothing and Non-parametric Regression. Technical report, Princeton University, http://data.princeton.edu/eco572/smoothing.pdf.

Ruppert, D. (2002). Selecting the Number of Knots for Penalized Splines. Journal of Computational and Graphical Statistics, 11(4):735-757.

Ruppert, D., Wand, M. P., and Carroll, R. J. (2003). Semiparametric Regression. Cambridge University Press, New Yor, NY. 
Sampford, M. R. (1967). On Sampling Without Replacement With Unequal Probabilities of Selection. Biometrika, 54.3(4):499-513.

Särndal, C. E., Swensson, B., and Wretman, J. (2003). Model assisted survey sampling. Springer Science \& Business Media.

Savitsky, T. and Toth, D. (2016). Bayesian Estimation Under Informative Sampling. Electronic Journal of Statistics, 10(1):1677-1708.

Schwarz, G. (1978). Estimating the Dimension of a Model. The Annals of Statistics, 6(2):461-464.

Shao, J. (1993). Linear Model Selection by Cross Validation. Journal of the American statistical Association, 88(422):486-494.

Statistics Canada (2016). A glimpse into history. Statistics Canada, , https://www.statcan.gc.ca/eng/about/history/index.

Statistics Canada (2018). Census Dictionary. Statistics Canada, https://www12.statcan.gc.ca/census-recensement/2016/ref/dict/az1-eng.cfm.

Sugasawa, S., Kubokawa, T., and Rao, J. N. K. (2018). Small Area Estimation via Unmatched Sampling and Linking Models. TEST, 27(2):407-427.

Torabi, M. and Rao, J. N. K. (2014). On Small Area Estimation Under a Sub-area Level Model. Journal of Multivariate Analysis, 127:36-55.

Vaida, F. and Blanchard, S. (2005). Conditional Akaike Information for Mixedeffects Models. Biometrika, 92(2):351-370.

Verret, F., Rao, J. N. K., and Hidiroglou, M. A. (2015). Model-based Small Area Estimation Under Informative Sampling. Survey Methodology, 41(2):333-347.

You, Y. and Rao, J. N. K. (2002). Small Area Estimation Using Unmatched Sampling and Linking Models. The Canadian Journal of Statistics, 30(1):3-15. 